University of Louisville

ThinkIR: The University of Louisville's Institutional Repository

Electronic Theses and Dissertations

1935

\title{
The air and vacuum drying of Prussian Blue.
}

David B. Ardern

University of Louisville

Follow this and additional works at: https://ir.library.louisville.edu/etd

Part of the Chemical Engineering Commons

\section{Recommended Citation}

Ardern, David B., "The air and vacuum drying of Prussian Blue." (1935). Electronic Theses and Dissertations. Paper 1674.

https://doi.org/10.18297/etd/1674

This Master's Thesis is brought to you for free and open access by ThinkIR: The University of Louisville's Institutional Repository. It has been accepted for inclusion in Electronic Theses and Dissertations by an authorized administrator of ThinkIR: The University of Louisville's Institutional Repository. This title appears here courtesy of the author, who has retained all other copyrights. For more information, please contact thinkir@louisville.edu. 
TIE

\section{AIR AKD VACOU:}

Drrxro

\section{OF PRUssian BIJB}


THE UNIVERSITY OF LOUISVILIX

\author{
A DISSERTA TIOK \\ SUBUITIED TO THE PACULTI \\ OF THE GRADUATE SCEOOL \\ IN PARTIAL FULPIIIUTM OP THB \\ REQUIREMENTS FOR THE DEAREE OP \\ MASTER OF SOIENGE \\ IN CHEYICAL BMOIMEERINO
}

By

DAVID B. ARDERN

1955 
TABLS OF CONTEMTS

IIST OF TABLES .............. Page

LIST OF CURNES _.............. 111

LIST OF PIOUREB _............. Ix

ACKMOWLEDGEMENT - . . . . . . . . $x$

INTRODUCTION $\ldots \ldots \ldots$

HISTORICAL _............... 3

PART I - AIR DRYIMO - - 12

THEORETICAL _............... 13

EXPERTMEITAL - . - . . . . . 19

A. APPARATUS and OPERATION - - - - 20

B. MTERIAL _............ 27

C. EXPERTIENTAL WORK - $\ldots \ldots$

RESULTS _. . . . . . . . . . 31

SUMMART $\ldots \ldots 65$

PART II - VACUUM DFYINO - 76

THEORETICAL _..............

EXPER IMENTAL _... $\ldots \ldots$

A. APPARA TUS and OPERATION _. - - 88

B. EXPERIMENTAL WORE _....... 87

RESULTS _. . . . . . . . . . . 91

SUnMARY .................. 170

ACKNOWLEDGEMENTS _............ 177

BIBLIOGRA PEY _.............. 178

13706 


\section{LISY OF MBLBS}

Table

Page

PART I - AIR DRYIHO

I TBUPERA TURE - HOMIDITI

CONDITIONS OP RUHS _.... 89

II 200 SHEET $-\operatorname{Run} 1 \ldots 33$

III MOISTURE CONTEIT DATA - RUn $1 \ldots 34$

IV RATS OF DRYINO DATA - Fun $1 \ldots 38$

$\checkmark \quad$ LOO SHEET - $\operatorname{Ran} 8 \ldots \ldots$

$\checkmark I$ MOISTURE CONTENT DATA - RUn $2 \ldots 48$

VII RATE OF DRYIMO DATA - RUA $2 \ldots-\ldots$

VIII LOO SHEET - $\operatorname{Rnn} 3 \ldots . \ldots$

IX MOISTURE CONTERT DATA - Run 5 - . 80

$x$ RATE OF DHINO DATA - Run $3 \ldots$...

XI LOG SHEET - RUA 4 . - . . . 57

XII MOISTURB CONTENT DATA - - . - - 68

XIII RATE OF DRYING DATA - RUan 4 - - 68

XIV COMPARISON OF AIR DRYINO DURVES - 69

PART II - VACUUA DRYIMO

XV TBYPERA TURE - VA CUUI

CONDITIOHS OF RUNS $\ldots . .-\ldots 8$

XVI LOO SHEET - $\operatorname{Ran} 1 \ldots 98$

XVII CAKK TEUPERATURE DATA - Run 1 -. 94 XVIII MOISTURE CONTENT DATA - Run $1 \ldots 97$ 


\section{LIST OF TABLES (Oont.)}

mbio

Page

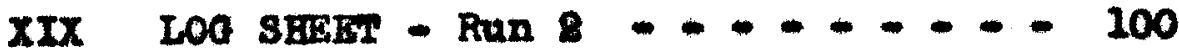

XX GAKE TEMPERA TURE DATA - RAn 8 - - 101

XXI NOISTJFE CONTEMT DATA - RAR \& - 104

XXII LOQ $8 \mathrm{HBR}$ - $\operatorname{Run} 3 \ldots 207$

XXIII CAIS TEMPERATORE DATA - Run 3- - 108

XXIV MOISTURE CONTERT DATA - Ran \$ - - 112

XXV LOR SHEEY - RUn 4 ........ 114

XXVI CAKE TEMPERATURE DATR - RUn 4 - - 116

XXVII MOISTURE CONTERT DATA - RUE 4 - - 127

XXVIII LOQ SHEEET - $\operatorname{Run} 5 \ldots 190$

XXIX CAXE TEMPERATURE DATA - RUn E - - 121

XXX MOISTUPE CONTENT DATA - Run 5 - - 124

XXXI LOA SHEBET - Run 6 ....... 187

XXXII CARE TEXPERATURE DATA - RUn $6 \ldots 288$

XXXIII MOISTORE CONTENT DATA - RUn $6 \ldots 251$

XXXIV LOG SHEET - Run $7 \ldots \ldots$. . . .

XXXV CAKE TEMPERATURE DATA - RUR $7 \ldots 235$

XXXVI HOISTURB CONTENT DATA - Run $7 \ldots 137$

yonII LOQ SHEET - RUR $8 \ldots 240$

XXXVIII CAKE TEMPERATURE DATA - RUA B - - 141

XXXIX MOISTURE CONTENT DATA - RUA $8 \ldots 243$ 


\section{LIST OF TABIES (Cont.)}

Table

Page

XI IOG SHEET - RUR 9 .......- 146

XII CAIE TEMPBRATURR DATA - RUA - - 147

XIII MOISTURE CONTENT DATA - Run 9 - 180

XLIII LOQ SHRET - RUR $10 \ldots 108$

XIIV GAKE TEMPERATORE DATA - Ran 10 - 184

XIV MOISTUFE CONTENT DATA - Run $20 \ldots-256$

XIVI LOO SHRET - Run $21 \ldots \ldots 9$

XEVII CAKE TEMPERATURE DATA - RAR 11- - 190

XDIII MOISTOFE CONTENT DATA - Ran $11 \ldots-168$

XLIX LOQ SHEES - $\operatorname{Rnn} 12 \ldots \ldots$

I CAIE TEMPERATORE DATA - RUA 12 - 266

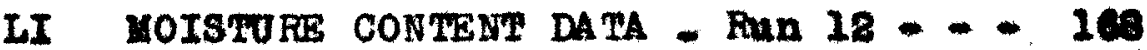




\section{IIST OP CORNES}

Carve

Page

\section{PART I - AIR DFIIHO}

I DRIIHG - RUR I ......... 37

II RATE OF DRYINO - Run 1 ..... 39

III DKYINO - Fun $2 \ldots 18$

IV RATE OF DRIINO - Run 8 ...... 47

$\checkmark$ DRYING - $\operatorname{RAR} 3 \ldots . . . . . . .58$

VI RATE OF DRIING - RUn $3 \ldots 5$

VII DRIINO - Ran 4 .......... 61

VIII RATE OF DRYINO - RUA $4 \ldots \ldots$

PART II - VACUUM DRIINO

IX CAKE THMPERATVRE - FUn $1 \ldots 96$

X DRYINO - Run $1 \ldots 90$

XI CAKE TEMPERATURE - FUn $8 \ldots 208$

XII DFIINO - $\operatorname{Run} 2 \ldots 106$

XIII CA BRE TEMPERATUFE - Run $3 \ldots 120$

XIV DRYIMG - Run $3 \ldots 123$

XV CAKE TEMPERATUKE - RUR 4 ... - 116

XVI DRYIHG - Run 4 . . . . . - 119

XVII CARE TEMPERA TURE - FUR 5 - . - 123

XVIII DRYINO - Run $5 \ldots \ldots 126$

XIX CAKE THUPERA TURE - RUU $6 \ldots 130$ 
LIST OF CURVES (Cont.)

curre

Page

XX DRYING - Fun $6 \ldots-\ldots+\ldots$

XXI CAKI TEXPERATURE - Run $7 \ldots-\ldots$

XXII DRYINo - $\operatorname{Ran} 7 \ldots \ldots+\ldots$

XXIII CAKE TEMPERA TURE - Fun $8-\cdots,--148$



XXV CAKE TEMPERATURE - Run $9-\ldots-\ldots-\infty$

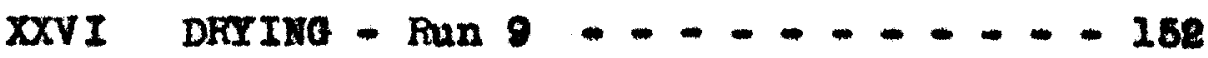

XXVII CAM TEMPERATURS - RAR $10 \ldots-\ldots$

XXVIII DRYINO - Man $10 \ldots \ldots-\ldots$

XXIX CAKE TBLPERATURE - RAR $11-\ldots-\ldots$

XXX DRTINO - $\operatorname{Ran} 11 \ldots+\ldots$

XXXI CAKE TEMPERATURE - RAR 12 - $\ldots$

XXXII DRYINO - $\operatorname{Ran} 12 \ldots \ldots+\ldots$ 


\section{LIST OF PIOURES}

F1 gure

$1{ }^{n} \mathrm{H}-\mathrm{W}^{n}$ CONDITIONER - . . . . . 81

2 DIAGRAMMATIC SKETCH OF AIR DRYER - - 28

3 BRISTOL RECORDINO PSYCHROLETER - - 25

4 comparison of ratz curves - . - . 68

5 VACUUM DRYER - . . . . . . . 83

6 SKETCH OF VACUUM DFYER _...... 85 


\title{
A CKNOWLEDAEMENT
}

\author{
The \\ Author Wishes \\ To Acknowledge His \\ Approciation
}

For All The KInd and Valuable Ald

Given by Dr. R. C. Ernat,

Who Directed This Researoh. 
IMPRODUCTIOA 
The purpose of thls Investigation was to determine the effoct of variod drying oondition upon the rate and meohanism of the drying of a coumerolal solld by alr and in racuum.

A1r drying of the aolld wan inrost1gated at various conditions of temperature and muidity. The particular condition obosen as to tomporature and humidity were woh as to chook and complote previoue experimental work performed by sobmied and Ardorn (1). It was also desired to oumarize the reanlts of this and the prerlous work (1), and then compare the drying rato curves obtained with the theoretical curves of Newman (2).

The vaourum drying of the col1d was - tudied under different conditions of temperature and vacuum. The dete and ourves obtained from the commorolel sol1d wore to be compared with the Inventigation of a thoorotical inert solid made by RIdgway (3).

The solid used in all of these teats was conmerolal Prussian Blue paint plgment. 
AIstonIaA 
The praction of Chonleal Enginoering involven prinoipally the oarrylng out of cortaln phyeloal oporation in the production of chomicale and wy othor almilar producte. Thoee phyeloal stepe are called the unlt oporations.

The maracture of product rosultins from ohemlanl reaction ofton Involves a series of those Individual unlt operatione, For example, in order to prepare a finlehod product, $1 t$ may bo necoseary for the raw materials to be ground, screened, dieselred, I1ltered, and aried, wh1lo the by-producte may be dist111ed, evaporated, and absorbed or orytalal1zod.

The development of these unlt operetions has been in atop with the rapld growth of the Cbomical Englnoering Industries. The oxiot theorotical character of those operations han not in mang cuses been Intently atudied. However, rocent Investigations have indicated that the basic principles involved in ang unit operation are the same, rogardies of 1te application, or the method by which it is carried out. 
One of those ongineoring operations is the unlt precess known as arying. "Drylng commonly refore to the removal of a Ilquid frem solld, the 11quid west frequentiy belng water. The removal of water from a sold in solution in termod oraporation, but thore 1s no distinotion between ovaporation and arying in crystallisation procosses. ordinarily drying refors to the removal of water from a o.11d when the water in present in relatively amall amounts" (4).

There are maxy types of dryere in commerolal use, the kind varylng widely according to the perticular propertios of the material to be dried. The actual claseification of the machines used in this unit operation is diffioult, but the simplest subdiviaion is into two clasees, atmospherio alr and vecuun dryora.

Alr argere are convenientiy afvided into three types (5):

1. The contimous eutcontic type. The material is conveyed meohanlcally through the aryer, of from compartmont to compartmont, whlle a transiorse clrculation of alr 1s usually malntalned. 
2. The tunnel typo. The material 1s passed through the aryer on trucks, whlle clrcalat1on of heated alr is malntained in a direction opposite to the movement of the material. Contruction permits the contlmove rooding of material at ono ond and removal at the opposito ond.

3. The compartment type. This aryer may bo an ontirely solf-containod unit, ae a dry k11n, all parts being rurnishod and sot up In the bullding lndependent of the bullding conetruotion. It may also bo almigly a room or compartment in the bullding, eltted with co11s, ran, or other drying apparatus need in the drying process.

The contimous and tunnol dryers are ofriclent only $1 f$ operated at uniform conditions over a long perlod of time. The compartment dryer adapt. 1tsolf well to maracturing batch units, whore operation is not a twenty-four hour achoduale.

The principle of operation of the a1r dryer is to pass hoated alf over the material bolng dr1ed. In the case of the compartment dryer this 
a1r 18 usually preheated by teas colls. In the contimous or tunnel aryer the flow of alr may be -1ther direct-current or counter-current. AccordIng to Hohbel (6) direct-current arying 1s thooretically more officlent than oounter-current.

Increased efflotenoy may be obtalned through more intimate contact of air and material according to J. Dom (7). Buck (5) warns agalnst too rapld drylag, which may result in warplng, cracking, or alscoloring of the material.

A wide variety of materiala are handied in alr aryers, the liat contalning auoh 1toms a: frult of 11 kinds, clay products, cotton linters, r1lma, rurs, h1des, palnts, lumber, matches, rage, mubber, coap, tobacco, wallboard, paatea, toxt1lo materiala, precipitatos, filtor cakes, etc.

Veoulu dryers way be divided into sholf dryers, for materials that do not noed to be stirrod while drylng; rotary dryera, for material which must be continuousiy stirred; and drum dryers, for substances which readily acquire a dry $111 \mathrm{~m}$ on the surface (8). 
Tho ra ouna aryer differe frem the alr dryer in that it operates at conulderably reduced proseure. It gives a very high rate of drging, usually at 20 temperatures, and is used for mater1als whloh would undergo oxldat1on, docemposition, or discoloration in alf dryors. A vacunm dryer w11 acoomplinh the same reaulte as an aryor of corrosponding capacity in a much shortor per1od of time, and at much lower team coste. However, the vecuna aryer has the higher cost of construct1on, and the added cost of oporating a vacum pump. Vacuum aryer are lmost invarlably operated Intermittently, one notable excoption belng



Vacuum drying was first used commerclally In 1881 (20), while the firet report of a cont1muous belt type vacuun dryor wa in 1906 (11). A study of the application and limitations of vacuum dryers was made by Lavett and Van Warlo (12), and of the mathomet1ce of vacuum drying by Martin (13).

Vacuram dryers are adapted to the drying of woch materiels as rubber of all kinds, palnts, dyes, extracts, pastes, gluo, soap, salta, albumens, 
staroh, glutin, rosin, regetables, fruits, sugara, chomicals, etc. (8).

Commerolal Pruesian Blue is the ferrocyanide of 1ron. To make 1t, a dilute solution of copperas (ferrous alfate), acldifled with mulfurio ac1d, is precipltated with potaselum forrooganide olution. After decanting the 11quor the white precipitate of ferroun-forrocyanido 10 oxidized with nitric acid, bloching powder and hydrochloric ac1d, or by exponure to a 1r (14).

There are number of varieties of Prussian Blue, all approxiately the same composit1on, though lightig different in ahade. They are made differently, and are known as steol Blue, Mllorl Blue, Bronze Blue, Antwerp Blue, Chlnese Blue, and Paria Blue (8).

A number of experimenter have disagreed as to the exnot oharacter of Pruselan Blue. AccordIng to Horman (15) and Muollor (16) the color 1s - ferrocjanide. Schuldt and Rassor (17) auggest that it is ferric forrocyanide, contalning some 
potessium in plece of the Iron, two mols of water In Intermolecular combination, and mell quant1ties or wdrated ferric axide in solld solution.

An X-ray analysis by Lovi (28) falled to detect a difference in crystalline atructure botween ferrous and ferric ferrocyanides. JuetinMnoller (19) atates that romotion with oxal10 acid Indicate the constitution to be forrio trirerroforrlejanlde, while reactions with potess ium hydroxide enggest formula of ferrous diferroforricyanide.

Prussian Blue is an excopt1onaliy etrong and permenent plgwent. It has extrome tinting powera, but 1 te transparenoy decreases its covering ablilty. The pure dry pigment has a bronze tinge, whioh disappora with docreasing particlo sire. The Inteneity of color depende upon the concentration of acid, temperature of procipitation, and amount of oxidant used. The dry plgment is very bard to grind, and is insoluble in dilute ocids and concentrated hydrochlor 10.

Morris (20) taten that the drying of the prese cakes is best carried out in a ourront 
of hot air. The product is a stronger and less "clndery" one than that obtalned from a veourm dryer.

Besides extensive applications as a paint plement, Pruesian Blue is used in mallpapor and In the toxt1le Industry (B) (14). A recont application of the plement has been in the procesaine of rubber (21), while it is also used in tho printing Industry (22) (2s), and in the proparation of photographic color plates (24). 
PART I

A IR DRY IHO 
THBORETICAL 
For each different material, under -pecific conditions of tomperature and mmidity, there is anid to be an equilibrium molsture content. This is the molsture content which the material will asume if loft for a ourfiolent length of time in contaot with air of those particular conditions.

If the Initial moleture content is higher than the equ111brium, the difference between the two 1s called the free molature content, and the removal of this froe moleture is the unit operation known as arying.

The thooretical mochaniam of the air drylng of solids has been studied by Lew1s (25), Sherwood (26), and Neman (2). According to their findings the drying of aolld takes place in two atages. The flrat may bo termed the constant rate perlod, whllo the second is known as the falling rate period.

The constant rate period appears when the amount of free molsture present is rolatively high. Water diffuses through the solld to the exposed surface, where the evaporation takes plece 
as rapldiy as drying condition pormit. Tho principlo resistance to the drying is in difrusion of vater vapor through the alr flim on the ourface of the olid. Since evaporation occurs on a surface wh1ch 1s kept wet, the temperature of the water and of the cake approaches the wot bulb tempereture of the drying air. During this period the rate of arying remaina a constant.

The point at which the constent rate period ends and the falling rate period commonces 1. known as the critical water oontent. Below this oritical water content the rate of drying curve is divided into the zone of unsaturated surface drying, and the zone whore Internal 11quid diffusion controls.

The zone of unsaturated surfece drying occure when diffulion of liguld within the solid 1. no longer repid enough to keep the entire surface wot. However, evaporetion continues to take place at the ourface, and the principle resiatance 1s in the air $11 \mathrm{~m}$. If the effoctive wotted surface during this zone is a linodr function of the water content, the drylag rate curro $\mathbf{w 1 1}$ be a 
straight 11no, though of a oonalderable nogative 1ope as ocmpared with the horizontel line or the constant rate period.

As the dry ing progresses, a second polnt 1. reached, bejond which difrusion through the solld 18 the controlling factor. The surface of the meterial haring prectioally reached tho oqu1l1brium content, the alr $\mathrm{rl}$ Im resistance becomes nogliglble. The controling factor, the difrueion resiatanoe, Increases at the amount of free moleture decresses, and the curve of the rate of drying is decreaningly negative as the solld approanes the equilibrium mo1sture content.

Daring the constant rate period and the unsaturated surface zono oraporation 18 occurlng at the surface. But at the beginning of the internal difrueion sone the ourfece of the col1d 10 pract10alis ary. Therefore, it is concelvable that, if the temperature is high onough, the plane or ovaporation may recede into the solid. If this happene, the rate of drying $\$ 111$ be oontrolled not only by rosistance to diffusion of liquid to tho plene of evaporation, but alvo by realatance to diffusion of the vapors through the remalning solid and the atr rilm. 
Constant conditions in alr drying moan constant temperature, humidity and alr velocity. The effect of varying these conditione may be road11y seon.

In most cases temperature has 11ttlo to do with the final equilibriun molsture content. It w111 affect only the rate of drylng. Inoreasing the temperature w111 hasten drying throughorat, as both evaporation at the aurfece and difrusion withIn the sol1d $\mathbf{w 1 1}$ be affected.

Increasing the mumidity dooreases tho differential between the water content of tho sol1d surface and the alr, theroby decreasing the driving force which is causing oraporation of the 11quid at the surface. Therofore, an Inoreased mumidity w111 decrease the rete of drylng during the constant rate period, and the unsaturated arface sone. It $\mathbf{w 1 1}$ not effect the sone of difrusion control.

The effect of Increaling the air velocity $\nabla 111$ be to decrease the alr f1Im at the surface. Thas a higher alr velocity 1111 increase the rate of drying long as the alr film resistance is an 
Important faotor, but w111 not affoct tho drying whon diffunion controls. However, the Inorease In rate of arying as air velocity inoreases is not a linear function.

Some ol1ds have tendency to oheck and warp on drying. These properties are given a material by the fact that it undergoes unequal enrinicage if the drying is not uniform. Some sol1de 180 how case-hardoning, the formation of a hard layer of driod material on the surface, which greatly hampers internal difrusion to the murface. These proporties are usually increased by Increased temperature and ir veloolty, but woh tendencles may be decressed by increasing the mumidity. 
BXPER IIENTAL 


\section{A. APPARATUS and OPERATIOD}

The dryer used in these toste was an "H - W" Conaltioner, mamuractured by the Industrial Dryor Corporation of Stanford, Connecticut. F1g. 1 18 a photograph of the dryer, while a diagram 1s given in F188, 2-A and 2-B.

In the operation of this dryor atmospher10 air 10 drawn in through the iniet pipe $A$, F1g. 2, by the ran at B. Pasaing over four sots of atoam colls in the hoating ohmmer $C$, the alr 1. brought to the desired temporature before passing under the bafrle wall $D$, Into the detribution obmiber E. Through the elots the hosted alf ia blown over the material on the traye in the arying ocmpartment $O$. From the outlet chmmber H, the alr may bo exheusted through the stack J, or reolroulated book through the dryer. The amounts of fresh and reclroulated alr are controlled by dampers $\mathrm{K}$ and L.

In ordêr to malntain the drying air at the desirod mamidity it was nocosanry to mix the als with steam by means of the nozsile $\mathrm{H}$. The steas was introduced fust before the fan in order 


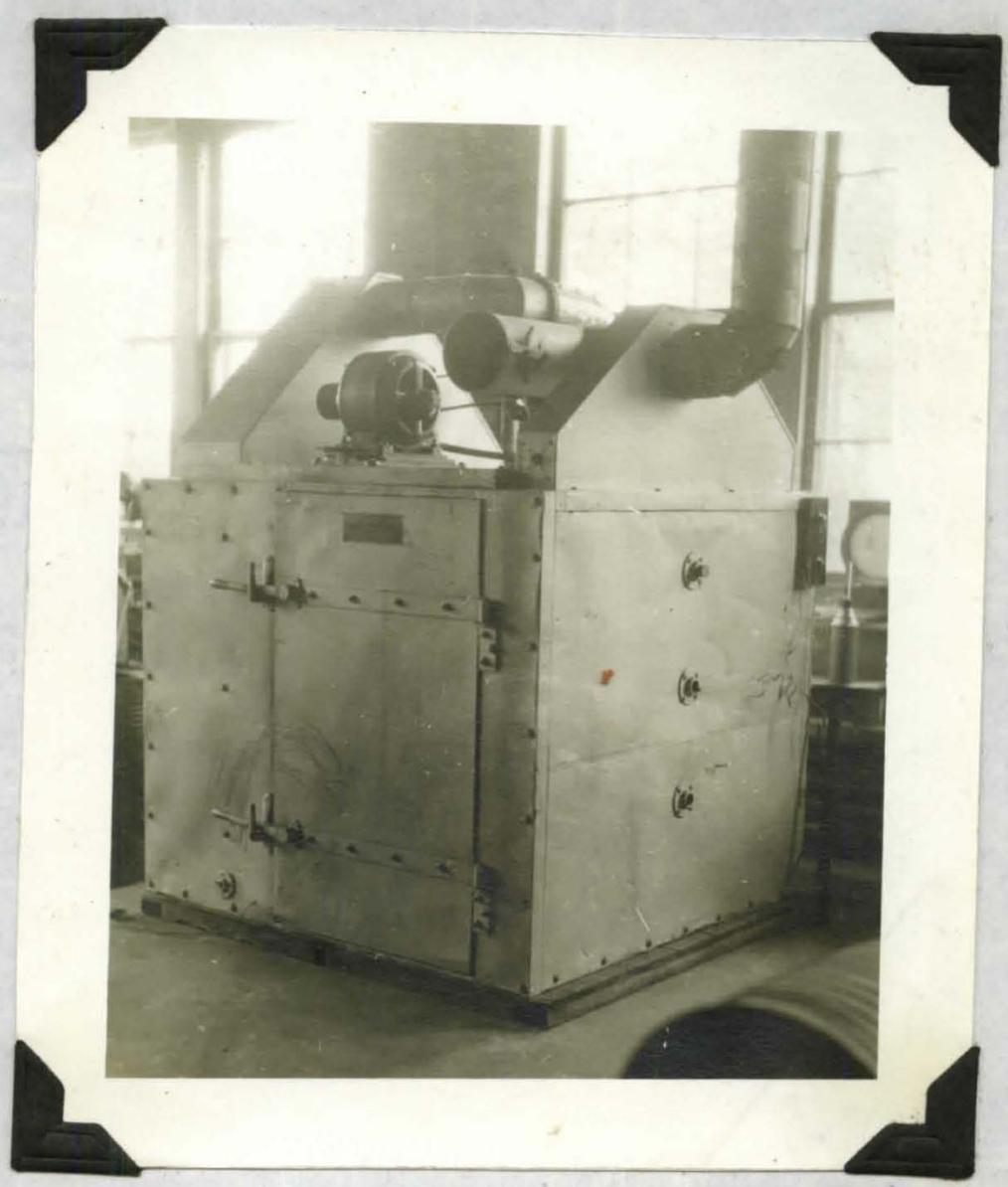

F1gure 1

"H - W" CONDITIONER 


\section{FIGURE 2-A}

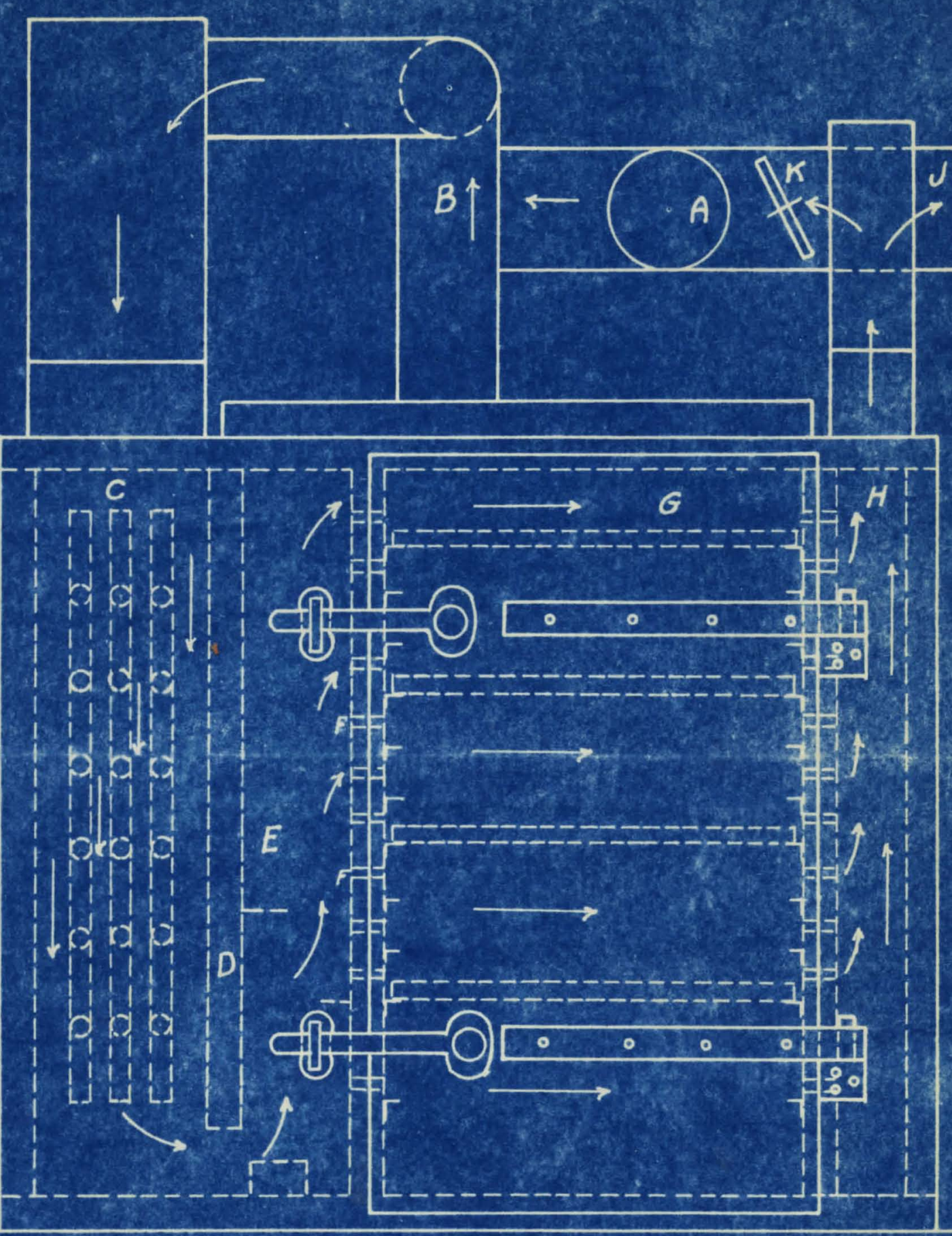

DIAGRAMATIC SKETCH OF DRIER 
FISURE 2-B

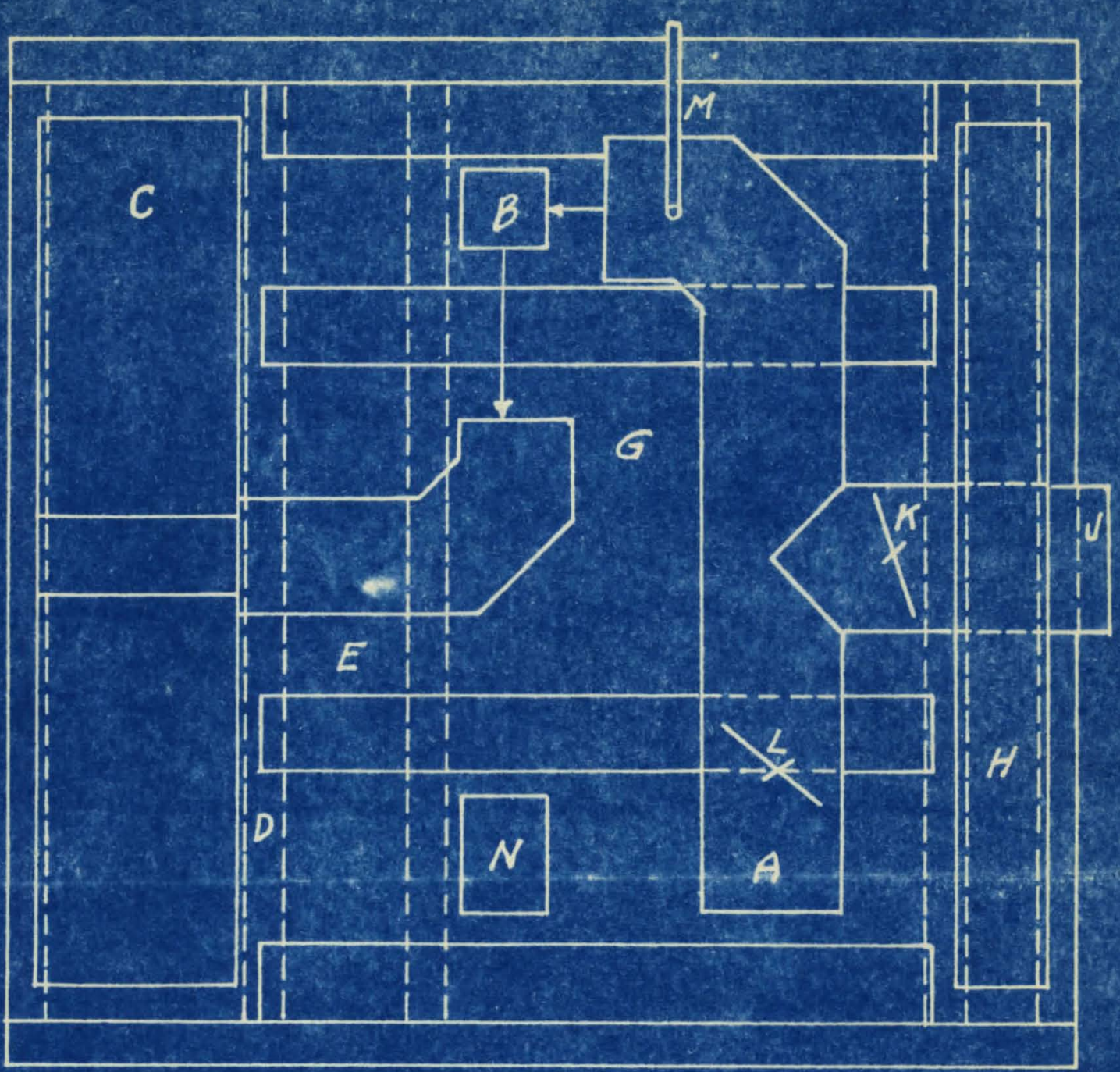

A-ENTERING AIR PIPE

B-FAN

C-HEATING CHAMEER

D-BAFFLE WALL

E-DISTRIBUTION CHAMOER

F-SLOTS

G-DRYING COMPARTMENT

H-OUTLET CHAMEER

$\checkmark$-OUTLET AIR PIPE

K- DAMPER

L-DAMPER

M-STEAM NOZZLE

N-MotoR

\section{DIAGRAMATIC SHETCH OF DRIER TOP VIEW}


to be cortaln of thorough mixing as the alr and ateng paseed through the ran.

Temperature and muldity date were obtainod at the inlot and outlot of the drgor by moans of wet and dry bulb thermemoters. A Briatol Recording Payohrometer, F18. 3, was ueed in controlling the condition of the alf. The pejohromoter wot and dxy bulbe wore placod at the bottcm of the Aletribution chamber B, F18, 8, while the water fount and rocording inutrument wore mounted on an Instrunent board at the rear of tho dryor.

The porcent reolrculation of the alr in the aryer was obtainod by moesuring the lnlot air relocity with en anemomoter whon the dempers were wot for sero roolroulation, and thon at ans othor doalrod eotting.

samples of the material dried wore taken In the carly parts of each run by monns of a cork boror, unt1l the materlal became too brittle, after whloh a knire wae used. The samples wore wolghod on a chaln-0mmatio balenos, and dried in a mall - lecbric drying oven. A large platform sale wa 
FIGURE 3

A - WET BULB THERMOMETER A'- DRY BULB THERMOMETER I- WATER INLET - water outlet D- Der buLB Recording PEN W- WET BULB RECORONA PEN

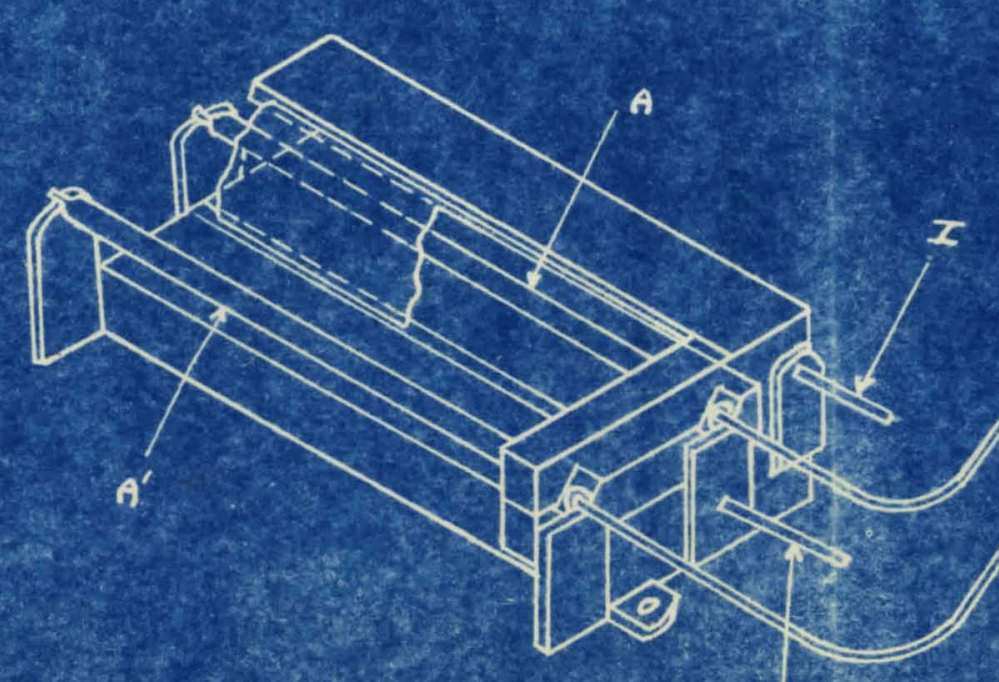

BRISTOL RECORDING PSYCHROMETER 
usod for welghing the pans and material. Steam was obtained from amall gas flred boller, which wa pert of the regular laboratory equipmont. 


\section{B. MATERIAL}

Tho material uned in those tosts was a standard comerclal Prussian Blue, Iurnishod by the Kentuokg Color and Chomlcal Compans of Loulev1110, Kentucks.

The plgment was mameotured by adding ammonlum sulfate and oulfurlo acld to forrous sulfate, and thon preclpitating with sodiun forrocjanide. 0xidation was carried out with odium ohlorate at sixty dogrees centigrade. Tho rosultant proolpitate was washed by decantation over a period of throe weoke, then riltered in a plate and Irame f12tor prese. Cakes from thla prose wore uned in those tosta.

The fliter cakes moasured twenty-two Inches square by bout one and ono-quartier Inches thlck. They had an initial molsture oontent of from forty-five to fifty percent water, and wolghod about twenty-F1ve pound oach whon wot.

A2l color was returnod to the Kentuck Color and Chemlenl compang after belng arlod. 
c. EXPERIURNTAL WORK

A total of cour run were wade at alfrorent temperature and muldity condition, as sbown in Tuble I. The riret two run wore performed as a obeck on the work of schmied and Andorn (1), while the last two were to obtaln addelonn 1 datin.

For each ran a total of twelve r11ter akes were used, two on each of als trayt. Tho traye vore placed on alternate abelves in tho aryor. With elx eapty traye ocoupsing the inter modicte oholved in order to satiefy the requirewents of aryer oonstruction.

In waking each ran the dryer wee rirut brought to the dosired condition of tempersture and muldity. The trays and matorial wore velghod and placed in the argor. Moloture eamplos wore taken from each of the elx traye at the atart of the mu, and at two-hour Intervals therearter throughout the run. Thoy were welghed imodiately. and then placed in the electric oven to ary for forty-01ght hours at temperature of seventy to elghty degreos Cont1grado. 
TABIS I

TEYPERATUFE - HUIIDITY CORDITIONS OF FUMS

$\begin{array}{ccc}\text { Run } & \text { Tempgraturo } & \text { munidity } \\ \text { No. } & 180 & 2.9 \text { (atmos.) } \\ 1 & 140 & 15 \\ 2 & 180 & 15 \\ 3 & 180 & 15 \\ 4 & & \end{array}$


The aryer was kopt at the dosired teaperature by controliling the steam in the colls. The necessary wet bulb temperature in ordor to give tho dosired manidity was dotormined from an monidity chart, and thon thia temperature mainteinod by rociroulating the alr and nixing it with stoam. These control tempereture were careruliy watohed on the recording psychrometer.

Readinge of the room temperature and Inlet and outlet wet and dry bulb thermometers were taken every two houra, and Inlet alr veloelty readings made three or four times during on oh run. At the ond of each ran the traye and material wore again weighed in order to determine tho total molsture lost durlng the mun. Each run lasted for a period of twenty-e1ght hours. 
RESULTS 
Tho date Included in the Log Sheota and in the Moleture Content Tables was taken directly from date kept during the rune in the Iaboratory.

Some value given in the molature oontent date are indicated an not belng used in caloulating the averages. Prolininary individunl Uolature Content ve Timo curves (not inoluded) wore plottod for each tray for caoh mun. The values ondted were those whloh were found to be conlderably off the goneral trend of the Individual curves. These epparentiy incorrect velues were attributed to the difficulty in alray obtalning a ropresentatire sample.

In calculatione for the rate of drying ourves for the varlous runs, vilues of the percent nolstures were reed directiy from the drylng curves. The weighte of the wot cakes at the beginntige of the rune were taken frose thelr respectivo $10 g$ aboets. 


\section{TABLS II}

\section{LOO BमKET}

Run 1

Departwent of Chomlcal Buglnoering

Speed Solentifle sehool

Univeralty of Loulovilie

DRYIMA OP PRUSSIAI BLJE

Pay ohrexoter Chart xo. 1

Deter $1 / 18 / 34$

Plant Record Data

Iaboratory Data

Name: Bronse Blue

Rx. Ho.: 4002

S11p VO: 607

Betoh No.: 28

Da to struck: $1 / 18 / 34$

Ne. Tray: Drled: 12

De to In: $1 / 17 / 34$

Bat oh Red.1 1/17/34

Batoh Rtd.1 $1 / 24 / 34$

Deylng Condition

Temperature! $180^{\circ} \mathrm{P}$.

Hamldity: $2.9 \%$ (Atmos.)

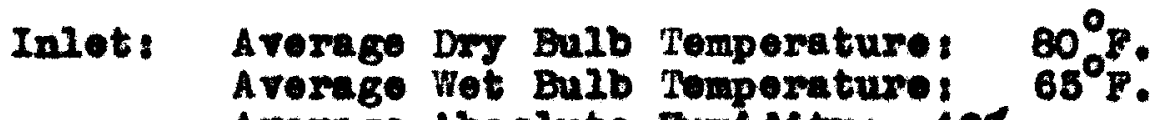

A verago Absolute muldity: 428

Outlot: Average Dry Bulb Tompernture: $184^{\circ} \mathrm{P}$. Arorage Wot Bulb Temperature: $180^{\circ} \mathrm{P}$. A rerage Absolute Bunlalty: $43 \%$

Average Room Temperature! $80^{\circ} \mathrm{F}$. Inlet AIr Velocity: $3060 \mathrm{ft} / \mathrm{min}$. foroent Roolrculetion: Vono

Recorded Peychromoter Chart Tompernturos: Dry Bulb: $180^{\circ} \mathrm{F}$. Wot Bulb: $96^{\circ} \mathrm{F}$.

Pan Data

\begin{tabular}{|c|c|c|c|}
\hline $\begin{array}{l}\text { Pan } \\
\text { No. }\end{array}$ & $\begin{array}{c}\text { Wt. of Cake } \\
\text { Before Dry Ing } \\
\text { Ibs. }\end{array}$ & $\begin{array}{l}\text { Wt. of Cake } \\
\text { After Drying } \\
\text { I ba. }\end{array}$ & $\begin{array}{l}\text { Wt. Koisture } \\
\text { Lost } \\
\text { Ibse. }\end{array}$ \\
\hline $\begin{array}{l}1 \\
8 \\
5 \\
4 \\
5 \\
6 \\
\text { Totala }\end{array}$ & $\begin{array}{r}46.00 \\
48.75 \\
48.25 \\
46.50 \\
46.25 \\
46.75 \\
268.50\end{array}$ & $\begin{array}{r}22.25 \\
21.75 \\
21.75 \\
21.25 \\
20.75 \\
21.60 \\
129.25\end{array}$ & $\begin{array}{r}23.75 \\
27.00 \\
26.50 \\
24.25 \\
26.50 \\
25.25 \\
155.25\end{array}$ \\
\hline
\end{tabular}


TABLB III

MOISTURS COKHEM DAA

$\operatorname{Ran} 1$



x Value cultted in average. 
TABLE III (Cont.)

MOISTURE CONTENT DATA

Run 1

\begin{tabular}{|c|c|c|c|c|}
\hline $\begin{array}{c}\text { Seraplo } \\
\text { Io. }\end{array}$ & Tray & $\begin{array}{c}\text { Wt. wot } \\
\text { sample } \\
\text { gmin. }\end{array}$ & $\begin{array}{l}\text { Moleture } \\
\text { Lont } \\
\text { gon. }\end{array}$ & $\begin{array}{l}\text { Percont } \\
\text { yolature }\end{array}$ \\
\hline 6 & $\begin{array}{l}1 \\
8 \\
5 \\
4 \\
5 \\
6\end{array}$ & $\begin{array}{l}5.600 \\
6.069 \\
5.814 \\
5.158 \\
7.488 \\
4.868\end{array}$ & $\begin{array}{l}1.516 \\
1.04 t \\
0.686 \\
0.755 \\
4.046 \\
1.086 \\
\text { Average }\end{array}$ & $\begin{array}{r}23.5 \\
\times 11.8 \\
14.4 \\
\times 54.2 \\
28.6 \\
20.2\end{array}$ \\
\hline 7 & $\begin{array}{l}1 \\
2 \\
8 \\
4 \\
5 \\
6\end{array}$ & $\begin{array}{l}5.569 \\
5.788 \\
5.867 \\
4.489 \\
5.389 \\
5.877\end{array}$ & $\begin{array}{l}0.884 \\
1.109 \\
1.776 \\
0.750 \\
2.165 \\
0.500 \\
\text { Average }\end{array}$ & $\begin{array}{r}15.9 \\
19.8 \\
30.3 \\
16.9 \\
\times 40.8 \\
11.0 \\
18.7\end{array}$ \\
\hline 8 & $\begin{array}{l}1 \\
2 \\
5 \\
4 \\
5 \\
6\end{array}$ & $\begin{array}{l}3.481 \\
4.730 \\
5.886 \\
2.381 \\
4.985 \\
3.610\end{array}$ & $\begin{array}{l}0.226 \\
0.436 \\
1.205 \\
0.037 \\
1.316 \\
0.086 \\
\text { Avertge }\end{array}$ & $\begin{array}{r}6.6 \\
9.2 \\
22.8 \\
1.6 \\
26.4 \\
1.6 \\
11.6\end{array}$ \\
\hline 9 & $\begin{array}{l}1 \\
2 \\
3 \\
4 \\
5 \\
6\end{array}$ & $\begin{array}{l}3.677 \\
3.486 \\
3.860 \\
3.659 \\
4.468 \\
3.030\end{array}$ & $\begin{array}{l}0.027 \\
0.038 \\
0.606 \\
0.110 \\
0.884 \\
0.055 \\
\text { Aterago }\end{array}$ & $\begin{array}{r}0.8 \\
1.1 \\
25.7 \\
3.3 \\
21.8 \\
1.2 \\
5.7\end{array}$ \\
\hline 10 & $\begin{array}{l}1 \\
2 \\
5 \\
4 \\
5 \\
6\end{array}$ & $\begin{array}{l}2.460 \\
4.675 \\
3.480 \\
4.045 \\
6.002 \\
8.384\end{array}$ & $\begin{array}{l}0.105 \\
0.032 \\
0.043 \\
0.048 \\
0.482 \\
0.094 \\
\text { Avers }\end{array}$ & $\begin{array}{l}4.3 \\
0.7 \\
1.8 \\
1.8 \\
8.0 \\
4.0 \\
3.2\end{array}$ \\
\hline
\end{tabular}

$x$ Value omitted in averago. 
TABLs III (Cont.)

MOIBNURE CORTEUT DATA

Bun 1

\begin{tabular}{|c|c|c|c|c|}
\hline $\begin{array}{c}\text { samplo } \\
\text { Fo. }\end{array}$ & In: & $\begin{array}{l}\text { Wt. Wot } \\
\text { sample } \\
\text { gant. }\end{array}$ & 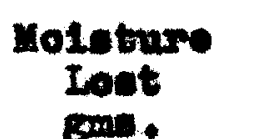 & $\begin{array}{l}\text { Poxoomt } \\
\text { Molngux }\end{array}$ \\
\hline 21 & $\begin{array}{l}2 \\
8 \\
3 \\
4 \\
5 \\
6\end{array}$ & $\begin{array}{l}4.045 \\
4.190 \\
3.928 \\
5.678 \\
4.366 \\
3.908\end{array}$ & $\begin{array}{l}0.001 \\
\text { L.08t } \\
0.888 \\
0.349 \\
0.009 \\
0.089 \\
\text { Average }\end{array}$ & $\begin{array}{l}0.0 \\
6.6 \\
6.3 \\
0.8 \\
0.8 \\
2.8\end{array}$ \\
\hline 18 & $\begin{array}{l}1 \\
8 \\
3 \\
4 \\
5 \\
6\end{array}$ & $\begin{array}{l}8.848 \\
3.787 \\
3.786 \\
3.039 \\
3.040 \\
2.984\end{array}$ & $\begin{array}{l}\text { Loot } \\
\text { Lost } \\
0.028 \\
0.020 \\
0.029 \\
0.026 \\
\text { Aferage }\end{array}$ & $\begin{array}{l}0.6 \\
0.3 \\
0.4 \\
0.5 \\
0.5\end{array}$ \\
\hline 13 & $\begin{array}{l}2 \\
8 \\
3 \\
4 \\
6 \\
6\end{array}$ & $\begin{array}{l}4.175 \\
5.867 \\
.668 \\
3.951 \\
5.131 \\
3.450\end{array}$ & $\begin{array}{l}\text { Leat } \\
0.006 \\
0.587 \\
0.305 \\
0.710 \\
0.018 \\
\text { A vermge }\end{array}$ & $\begin{array}{r}0.1 \\
\times 12.6 \\
7.7 \\
\times 14.0 \\
0.6 \\
+2.8\end{array}$ \\
\hline 14 & $\begin{array}{l}2 \\
2 \\
3 \\
4 \\
6 \\
6\end{array}$ & $\begin{array}{l}2.088 \\
5.689 \\
5.340 \\
4.917 \\
4.611 \\
5.469\end{array}$ & $\begin{array}{l}\text { Lott } \\
0.008 \\
0.058 \\
0.010 \\
0.046 \\
0.058 \\
\text { Arerage }\end{array}$ & $\begin{array}{l}0.8 \\
1.0 \\
0.2 \\
1.0 \\
0.6 \\
0.6\end{array}$ \\
\hline 15 & $\begin{array}{l}1 \\
8 \\
3 \\
4 \\
6\end{array}$ & $\begin{array}{l}4.674 \\
4.667 \\
4.566 \\
4.407 \\
4.510 \\
4.567\end{array}$ & $\begin{array}{l}\text { Loet } \\
\text { Lost: } \\
0.297 \\
0.010 \\
0.029 \\
0.017 \\
\text { Average }\end{array}$ & $\begin{array}{l}6.5 \\
0.9 \\
0.7 \\
0.4 \\
0.1\end{array}$ \\
\hline
\end{tabular}

I Valuo onltted in everage. 


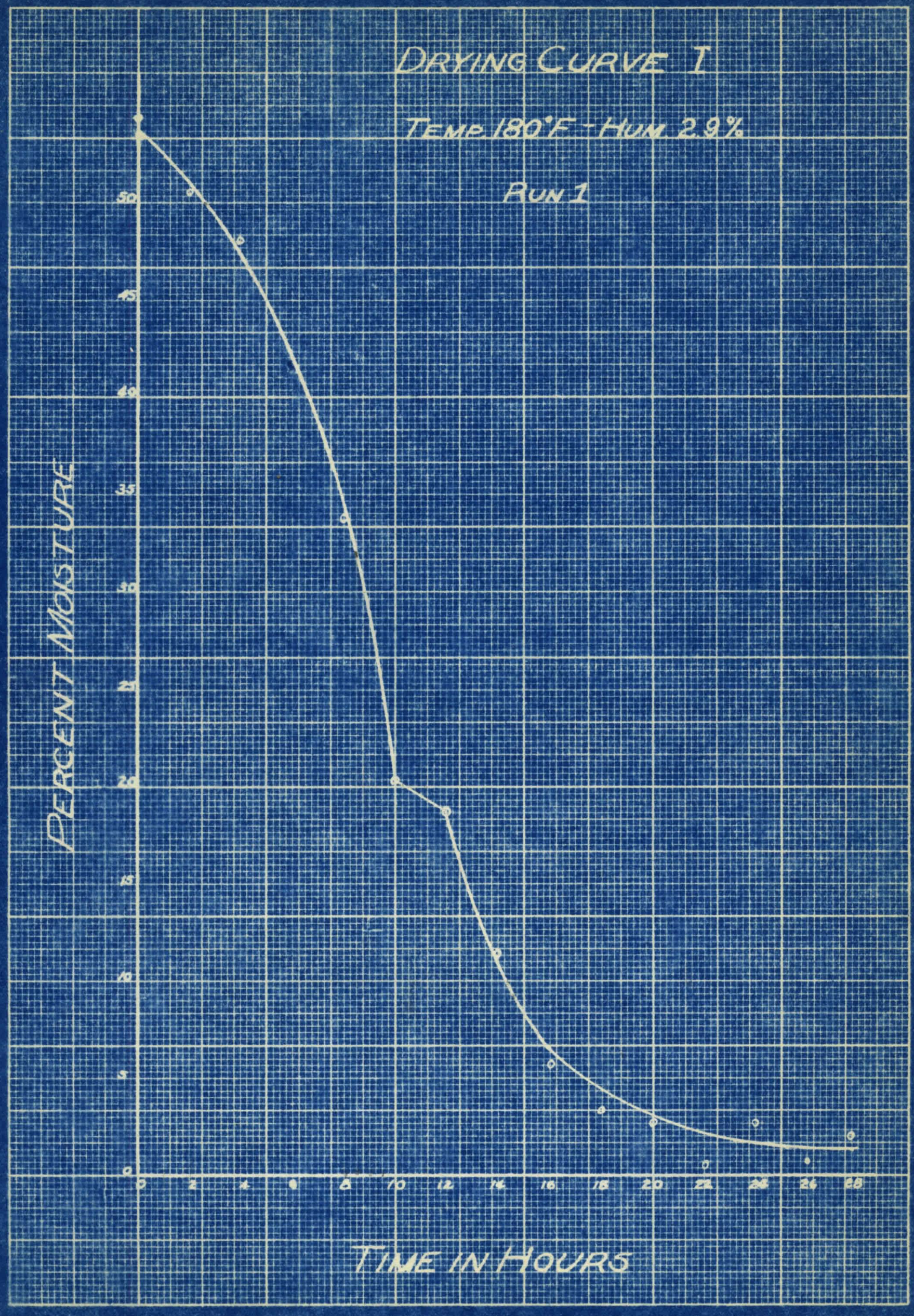




\section{TABLE IV}

RATE OF DRTIMO DATA

$\operatorname{man} 1$

\begin{tabular}{|c|c|c|c|c|c|c|c|}
\hline $\begin{array}{c}\text { Sample } \\
\text { No. }\end{array}$ & $\begin{array}{c}\text { Percent } \\
\text { Molsture } \\
\text { (From Curve) }\end{array}$ & $\begin{array}{l}\text { Wt. Het } \\
\text { Cako } \\
\text { Iba. }\end{array}$ & $\begin{array}{l}\text { Wt. Day } \\
\text { Cake } \\
\text { Ibs. }\end{array}$ & $\begin{array}{l}\text { Molsture } \\
\text { Present } \\
\text { Ibs. }\end{array}$ & $\begin{array}{l}\text { Moletwe } \\
\text { Loet } \\
\text { Ibe. }\end{array}$ & 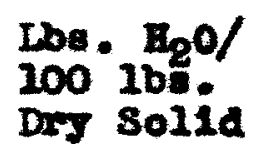 & $\begin{array}{l}\text { Lbs. Ho } \\
\text { Evaporated } \\
\text { /hr./aq.rt / }\end{array}$ \\
\hline $\begin{array}{r}1 \\
2 \\
3 \\
4 \\
5 \\
6 \\
7 \\
8 \\
9 \\
10 \\
11 \\
12 \\
13 \\
14 \\
15\end{array}$ & $\begin{array}{l}55.8 \\
51.0 \\
47.8 \\
41.8 \\
34.3 \\
20.3 \\
18.7 \\
10.8 \\
6.4 \\
4.4 \\
3.1 \\
2.8 \\
1.6 \\
1.4 \\
1.3\end{array}$ & $\begin{array}{l}285 \\
266 \\
248 \\
284 \\
109 \\
164 \\
161 \\
146 \\
110 \\
137 \\
135 \\
134 \\
135 \\
132 \\
132\end{array}$ & $\begin{array}{l}131 \\
131 \\
131 \\
131 \\
131 \\
131 \\
131 \\
231 \\
131 \\
131 \\
131 \\
151 \\
131 \\
131 \\
131\end{array}$ & $\begin{array}{r}289 \\
236 \\
117 \\
95 \\
68 \\
38 \\
30 \\
15 \\
9 \\
6 \\
4 \\
3 \\
8 \\
1 \\
1\end{array}$ & $\begin{array}{r}17 \\
18 \\
24 \\
25 \\
35 \\
3 \\
25 \\
6 \\
5 \\
2 \\
1 \\
1 \\
1 \\
0\end{array}$ & $\begin{array}{r}116.0 \\
103.2 \\
89.3 \\
7.0 \\
51.8 \\
25.8 \\
22.8 \\
11.4 \\
8.8 \\
4.6 \\
3.1 \\
2.3 \\
1.5 \\
0.8 \\
0.8\end{array}$ & $\begin{array}{l}-5.211 \\
0.211 \\
0.253 \\
0.298 \\
0.310 \\
0.434 \\
0.037 \\
0.186 \\
0.074 \\
0.057 \\
0.025 \\
0.012 \\
0.018 \\
0.018 \\
0.000\end{array}$ \\
\hline
\end{tabular}




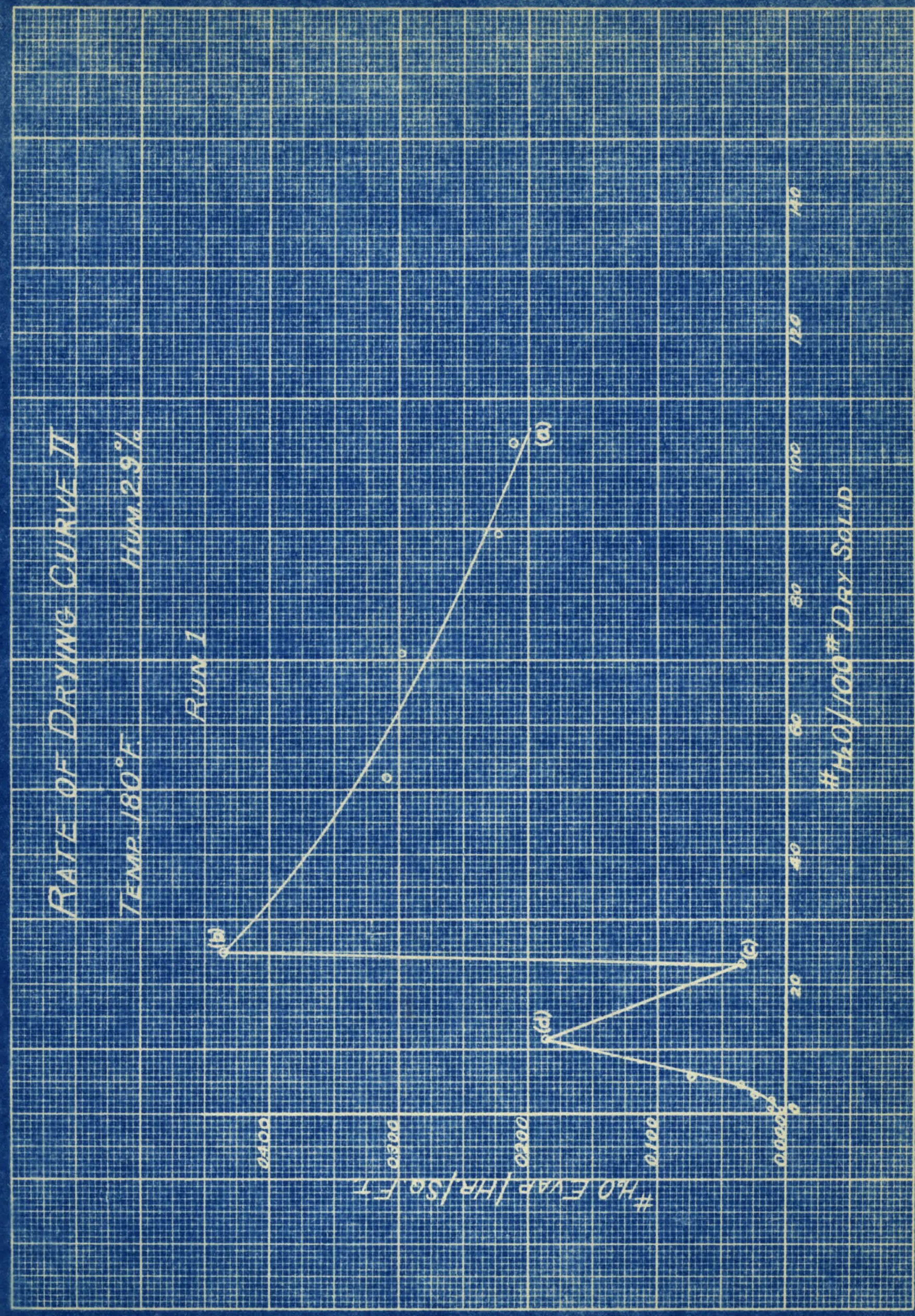


The ary ing conditions for Run 1 wore a content temperature of $280^{\circ} \mathrm{F}$, and monidity of approximetely 8.9\%. The oupply of als wes taken at atmospheric oonditions from the room, without rocirouletion of muldification with steam.

Drylns Curve I how apld decrease in molature content during the rirst ten hours of the sun. The decrease during the next two houra was very 10r, aftor which tho moloturo content again decreased rery sapldif for about four hours, and thon approached an equilibirum condition with the drying a1r.

Rate of Drying curve II Indicates an increase in the rate of drylng during the firat twelve houre (a to b). Daring the next two hours the rate of arying decreases to a low value (b to o), again 1ncreases (o to d), and thon drops to soro. 
TABIB V

LOQ SHEBT

Fun 8

Department of Chemloul Englneoring

Speed sclentiflo school

Univereity of Loulavilio

DRYING OF PRUSSIAN BLUE

Paychromoter Chert Ho, 2

Date: $1 / 25 / 34$

Plant Rocord Data

Laboratory Data

Name: Bronze Blue

Rx. No.: 4002

SIIp No.: 607

Butoh Ho.: 19

Date Struck: $1 / 17 / 34$

No, Trays Dried: 20

Date In: $1 / 24 / 34$

Batch Red.: $2 / 84 / 34$

Batch Rtd.: 2/14/34

Drying Conditions

Teaperature: $140^{\circ} \mathrm{C}$.

Humidity: 15\%

Inlet: Average Dry Bulb Temperature: ${ }^{80}{ }^{\circ} \mathrm{F}$. Average Wet Bulb Temperature:

Outlot: A verage Dry Bulb Tomperature: $120 \% \mathrm{~F}$. Average Wet Bulb Temperature: $104 \mathrm{~F}$. Average Absolute Fnonidity $56 \%$

A verage Rocm Temperature: $81^{\circ} \mathrm{F}$. Inlot Ais Velocity: $1143 \mathrm{ft} . / \mathrm{min}$. Percent Reolreulation: 63\%

Reoorded Paychrometor chart Tomperatures: Dry Bulb: $140^{\circ} \mathrm{P}$.

Pan Data

\begin{tabular}{|c|c|c|c|}
\hline $\begin{array}{l}\text { Pan } \\
\text { No. }\end{array}$ & $\begin{array}{c}\text { Wt. of Cake } \\
\text { Before Drying } \\
\text { lbs. }\end{array}$ & $\begin{array}{l}\text { Wt. of Cake } \\
\text { After Drying } \\
\text { IDs. }\end{array}$ & $\begin{array}{l}\text { Wt. Mo1sture } \\
\text { Iost } \\
\text { Ibs. }\end{array}$ \\
\hline 1 & $\begin{array}{r}50.25 \\
48.75 \\
50.75 \\
47.75 \\
51.50 \\
47.00 \\
\quad 296.00\end{array}$ & $\begin{array}{r}29.00 \\
28.00 \\
28.50 \\
24.00 \\
28.50 \\
25.50 \\
165.50\end{array}$ & $\begin{array}{r}21.25 \\
20.75 \\
22.25 \\
23.75 \\
23.00 \\
21.50 \\
132.50\end{array}$ \\
\hline
\end{tabular}


TABLE VI

MOTSTVRE COHTEN DA

$\operatorname{Ran} 8$

\begin{tabular}{|c|c|c|c|c|}
\hline $\begin{array}{c}\operatorname{sanp} 10 \\
10 .\end{array}$ & Ret & $\begin{array}{c}\text { Wt. Wot } \\
\text { Senge } 10 \\
\text { gons. }\end{array}$ &  & $\begin{array}{l}\text { Parount } \\
\text { nele bure }\end{array}$ \\
\hline 1 & $\begin{array}{l}1 \\
2 \\
8 \\
4 \\
5 \\
6\end{array}$ & $\begin{array}{l}5.518 \\
5.381 \\
5.758 \\
4.787 \\
6.083 \\
5.465\end{array}$ & $\begin{array}{l}2.686 \\
2.846 \\
2.805 \\
2.798 \\
8.986 \\
2.477 \\
\text { Rrem } 80\end{array}$ & $\begin{array}{l}48.3 \\
47.9 \\
48.7 \\
47.1 \\
48.3 \\
45.4 \\
47.6\end{array}$ \\
\hline $\mathbf{8}$ & $\begin{array}{l}1 \\
2 \\
3 \\
4 \\
5 \\
6\end{array}$ & $\begin{array}{l}6.039 \\
5.438 \\
5.459 \\
5.189 \\
5.707 \\
5.253\end{array}$ & $\begin{array}{l}2.080 \\
2.650 \\
2.591 \\
2.258 \\
\text { Lost } \\
\text { Lost } \\
\text { Average }\end{array}$ & $\begin{array}{l}51.2 \\
49.4 \\
47.4 \\
43.9 \\
47.7\end{array}$ \\
\hline $\mathfrak{3}$ & $\begin{array}{l}1 \\
8 \\
3 \\
4 \\
6 \\
6\end{array}$ & $\begin{array}{l}6.217 \\
5.186 \\
5.590 \\
5.428 \\
5.286 \\
4.688\end{array}$ & $\begin{array}{l}2.868 \\
2.231 \\
2.465 \\
2.399 \\
2.809 \\
2.038 \\
\text { Atorago }\end{array}$ & $\begin{array}{l}46.0 \\
43.6 \\
44.6 \\
44.3 \\
47.8 \\
43.4 \\
45.0\end{array}$ \\
\hline 4 & $\begin{array}{l}1 \\
2 \\
3 \\
4 \\
5 \\
6\end{array}$ & $\begin{array}{l}6.304 \\
5.582 \\
5.685 \\
4.867 \\
5.417 \\
3.789\end{array}$ & $\begin{array}{l}2.647 \\
2.312 \\
2.260 \\
1.845 \\
2.541 \\
1.547 \\
\text { A7ernge }\end{array}$ & $\begin{array}{l}42.0 \\
41.8 \\
40.0 \\
40.0 \\
46.8 \\
35.6 \\
41.1\end{array}$ \\
\hline 6 & $\begin{array}{l}1 \\
2 \\
3 \\
4 \\
5 \\
6\end{array}$ & $\begin{array}{l}6.308 \\
5.498 \\
4.978 \\
5.348 \\
5.785 \\
6.407\end{array}$ & $\begin{array}{r}2.897 \\
2.285 \\
1.865 \\
2.014 \\
2.340 \\
2.091 \\
\text { Average }\end{array}$ & $\begin{array}{l}41.2 \\
42.1 \\
37.6 \\
37.7 \\
41.9 \\
38.6 \\
38.6\end{array}$ \\
\hline
\end{tabular}




\section{TABIE VI (Cont.) \\ YOISTURE CONTERT DATA \\ Run 2}

\begin{tabular}{|c|c|c|c|c|}
\hline $\begin{array}{c}\text { Samplo } \\
\text { No. }\end{array}$ & Trat & $\begin{array}{c}\text { wt. Wot } \\
\text { sampio } \\
\text { gave. }\end{array}$ & $\begin{array}{l}\text { roieture } \\
\text { Loat } \\
\text { gas. }\end{array}$ & $\begin{array}{l}\text { Peroent } \\
\text { moleture }\end{array}$ \\
\hline B & $\begin{array}{l}2 \\
2 \\
5 \\
4 \\
5 \\
6\end{array}$ & $\begin{array}{l}7.288 \\
6.005 \\
4.998 \\
7.878 \\
6.891 \\
3.884\end{array}$ & $\begin{array}{l}2.688 \\
1.694 \\
1.697 \\
2.369 \\
2.320 \\
1.208 \\
\text { A.70248 }\end{array}$ & $\begin{array}{l}37.2 \\
33.0 \\
35.0 \\
32.6 \\
33.7 \\
31.8 \\
33.8\end{array}$ \\
\hline$\eta$ & $\begin{array}{l}1 \\
2 \\
8 \\
4 \\
5 \\
6\end{array}$ & $\begin{array}{l}5.711 \\
6.001 \\
6.234 \\
7.184 \\
6.189 \\
6.189\end{array}$ & $\begin{array}{l}1.851 \\
1.975 \\
8.206 \\
2.790 \\
2.488 \\
8.488 \\
\text { A7orago }\end{array}$ & $\begin{array}{r}32.4 \\
38.0 \\
35.4 \\
\times 38.4 \\
\times 40.8 \\
\times 40.2 \\
35.8\end{array}$ \\
\hline 8 & $\begin{array}{l}2 \\
2 \\
5 \\
4 \\
5 \\
6\end{array}$ & $\begin{array}{l}5.780 \\
4.081 \\
6.955 \\
5.094 \\
5.808 \\
5.098\end{array}$ & $\begin{array}{l}1.738 \\
1.283 \\
2.015 \\
1.258 \\
1.735 \\
1.167 \\
\text { A roxuge }\end{array}$ & $\begin{array}{l}29.9 \\
29.0 \\
29.0 \\
24.8 \\
29.8 \\
22.9 \\
21.6\end{array}$ \\
\hline 8 & $\begin{array}{l}1 \\
8 \\
5 \\
4 \\
5 \\
6\end{array}$ & $\begin{array}{l}5.750 \\
6.671 \\
5.981 \\
5.885 \\
6.858 \\
4.764\end{array}$ & $\begin{array}{l}1.507 \\
1.682 \\
1.356 \\
2.371 \\
1.894 \\
0.580 \\
\text { Arerage }\end{array}$ & $\begin{array}{l}27.8 \\
25.8 \\
22.4 \\
28.3 \\
27.6 \\
10.8 \\
22.6\end{array}$ \\
\hline 10 & $\begin{array}{l}1 \\
8 \\
8 \\
4 \\
5 \\
6\end{array}$ & $\begin{array}{l}6.273 \\
6.972 \\
7.283 \\
6.244 \\
6.491 \\
5.460\end{array}$ & $\begin{array}{l}1.548 \\
1.875 \\
1.581 \\
0.676 \\
0.984 \\
0.808 \\
\text { Arorago }\end{array}$ & $\begin{array}{l}21.4 \\
18.3 \\
21.3 \\
11.0 \\
15.8 \\
11.0 \\
16.4\end{array}$ \\
\hline
\end{tabular}

$x$ Valuo ondted in arorage. 


\section{TABLE VI (Oont.) \\ LOISTURE OONTENT DATA}

Ran 2

\begin{tabular}{|c|c|c|c|c|}
\hline $\begin{array}{c}\text { Senpe } 20 \\
\text { no. }\end{array}$ & Ho. & $\begin{array}{c}\text { Wt. Wet } \\
\text { semple } \\
\text { grms. }\end{array}$ & $\begin{array}{l}\text { Noletrue } \\
\text { Lent } \\
\text { gent. }\end{array}$ & $\begin{array}{l}\text { Poreont } \\
\text { Holetnit }\end{array}$ \\
\hline 21 & $\begin{array}{l}1 \\
8 \\
3 \\
4 \\
5 \\
6\end{array}$ & $\begin{array}{l}4.486 \\
6.889 \\
3.807 \\
4.881 \\
5.688 \\
5.044\end{array}$ & $\begin{array}{l}0.005 \\
0.085 \\
0.601 \\
0.678 \\
1.078 \\
0.086\end{array}$ & $\begin{array}{r}20.4 \\
13.7 \\
15.6 \\
14.0 \\
18.9 \\
12.4 \\
15.8\end{array}$ \\
\hline 12 & $\begin{array}{l}1 \\
8 \\
3 \\
4 \\
8 \\
6\end{array}$ & $\begin{array}{l}6.284 \\
7.559 \\
6.382 \\
5.409 \\
8.279 \\
4.744\end{array}$ & $\begin{array}{l}0.694 \\
1.104 \\
0.688 \\
0.498 \\
1.438 \\
0.868 \\
\text { A7oximge }\end{array}$ & $\begin{array}{r}11.1 \\
14.7 \\
10.6 \\
0.8 \\
17.4 \\
5.5 \\
11.4\end{array}$ \\
\hline 18 & $\begin{array}{l}1 \\
8 \\
3 \\
4 \\
6 \\
6\end{array}$ & $\begin{array}{l}6.850 \\
5.964 \\
6.168 \\
5.855 \\
5.748 \\
5.198\end{array}$ & $\begin{array}{l}0.658 \\
0.378 \\
0.550 \\
0.650 \\
0.858 \\
0.178 \\
\text { A.0mge }\end{array}$ & $\begin{array}{r}9.6 \\
9.5 \\
8.9 \\
11.1 \\
15.6 \\
3.3 \\
9.7\end{array}$ \\
\hline 24 & $\begin{array}{l}1 \\
2 \\
5 \\
4 \\
5 \\
6\end{array}$ & $\begin{array}{l}6.544 \\
6.274 \\
4.865 \\
3.175 \\
3.808 \\
4.544\end{array}$ & $\begin{array}{l}0.584 \\
0.867 \\
0.800 \\
0.086 \\
0.868 \\
0.164 \\
\text { Avernge }\end{array}$ & $\begin{array}{r}8.9 \\
9.0 \\
10.3 \\
\times \quad 8.7 \\
7.0 \\
3.0 \\
1.6\end{array}$ \\
\hline 15 & $\begin{array}{l}1 \\
2 \\
5 \\
4 \\
6 \\
6\end{array}$ & $\begin{array}{l}5.856 \\
5.885 \\
4.643 \\
6.358 \\
6.157 \\
4.496\end{array}$ & $\begin{array}{l}0.488 \\
0.580 \\
0.385 \\
0.618 \\
0.896 \\
0.180 \\
\text { A792ago }\end{array}$ & $\begin{array}{r}8.3 \\
8.7 \\
8.3 \\
9.6 \\
\times 14.5 \\
2.7 \\
7.6\end{array}$ \\
\hline
\end{tabular}

$x$ Value onitted in arerago. 


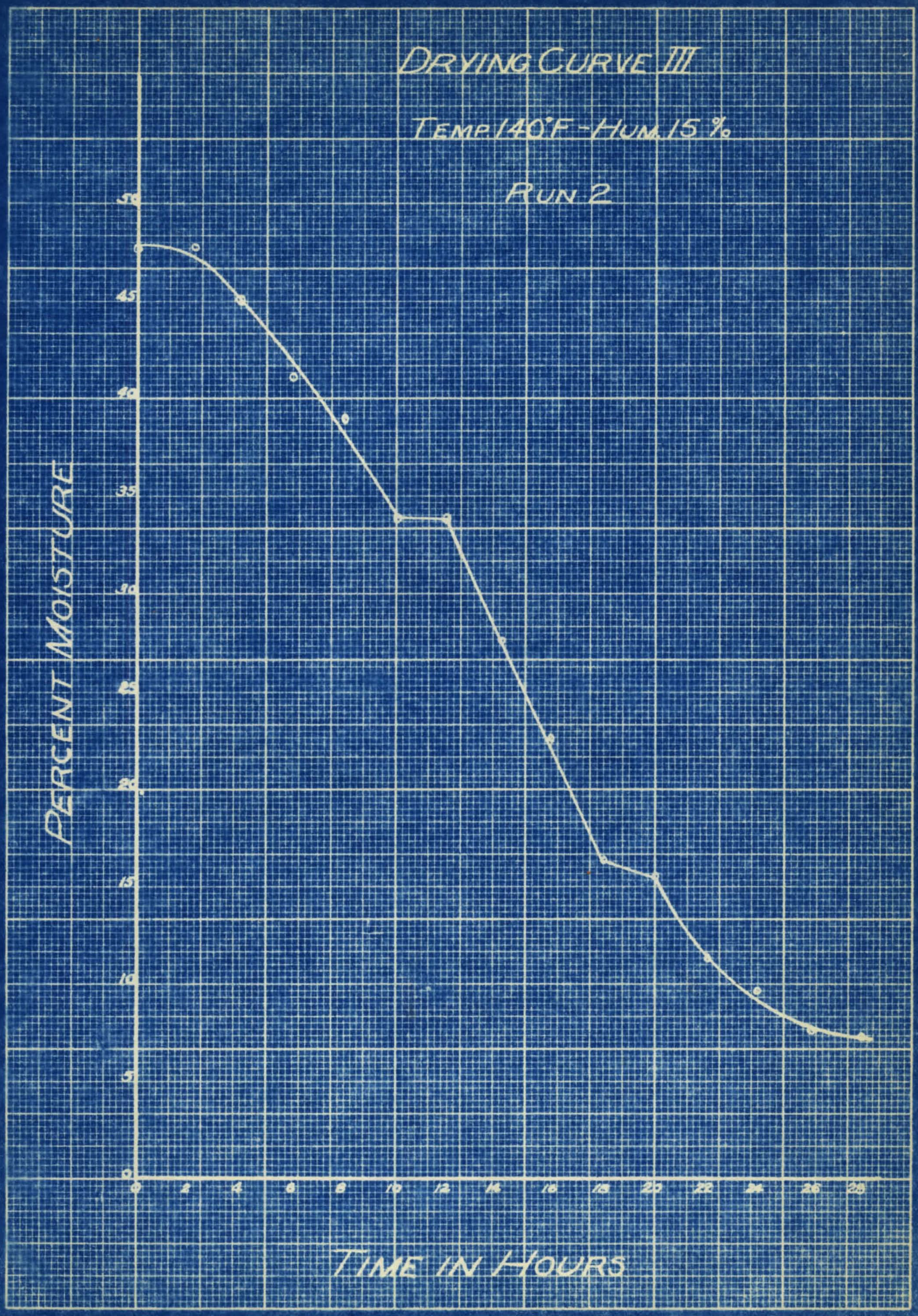


PABID VII

RATE OF DRIIVO DATA

Fine 2

\begin{tabular}{|c|c|c|c|c|c|c|c|}
\hline $\begin{array}{c}\text { Sample } \\
\text { 10. }\end{array}$ & $\begin{array}{c}\text { Percont } \\
\text { Moletnre } \\
\text { (Prom Curve) }\end{array}$ & $\begin{array}{l}\text { Wt. Wot } \\
\text { Cako } \\
\text { Ibs. }\end{array}$ & $\begin{array}{l}\text { wt. Der } \\
\text { Calce } \\
\text { Ibe. }\end{array}$ & $\begin{array}{l}\text { Wolature } \\
\text { Present } \\
\text { Ibe. }\end{array}$ & $\begin{array}{l}\text { Moleture } \\
\text { Lost } \\
\text { Ibs. }\end{array}$ & 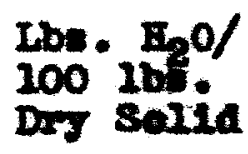 & 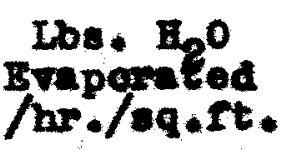 \\
\hline $\begin{array}{r}1 \\
2 \\
3 \\
4 \\
5 \\
6 \\
7 \\
8 \\
9 \\
10 \\
11 \\
12 \\
13 \\
14 \\
15\end{array}$ & $\begin{array}{r}47.8 \\
7.3 \\
78.0 \\
41.7 \\
38.1 \\
33.8 \\
3.8 \\
27.5 \\
21.8 \\
16.3 \\
15.4 \\
11.4 \\
9.1 \\
7.8 \\
7.2\end{array}$ & $\begin{array}{l}296 \\
293 \\
281 \\
265 \\
240 \\
235 \\
235 \\
213 \\
197 \\
185 \\
182 \\
174 \\
170 \\
167 \\
166\end{array}$ & $\begin{array}{l}154 \\
154 \\
144 \\
154 \\
154 \\
154 \\
154 \\
154 \\
154 \\
154 \\
154 \\
154 \\
154 \\
154 \\
154\end{array}$ & $\begin{array}{l}148 \\
130 \\
127 \\
121 \\
95 \\
79 \\
79 \\
89 \\
48 \\
31 \\
28 \\
20 \\
16 \\
13 \\
12\end{array}$ & $\begin{array}{r}3 \\
38 \\
16 \\
16 \\
16 \\
0 \\
80 \\
16 \\
18 \\
3 \\
8 \\
4 \\
3 \\
1\end{array}$ & $\begin{array}{l}92.8 \\
90.8 \\
82.4 \\
72.1 \\
61.7 \\
51.3 \\
51.3 \\
36.3 \\
87.9 \\
80.1 \\
18.2 \\
13.0 \\
10.4 \\
6.4 \\
5.8\end{array}$ & $\begin{array}{l}0.057 \\
0.037 \\
0.140 \\
0.198 \\
0.198 \\
0.198 \\
0.000 \\
0.218 \\
0.198 \\
0.149 \\
0.037 \\
0.090 \\
0.080 \\
0.037 \\
0.018\end{array}$ \\
\hline
\end{tabular}




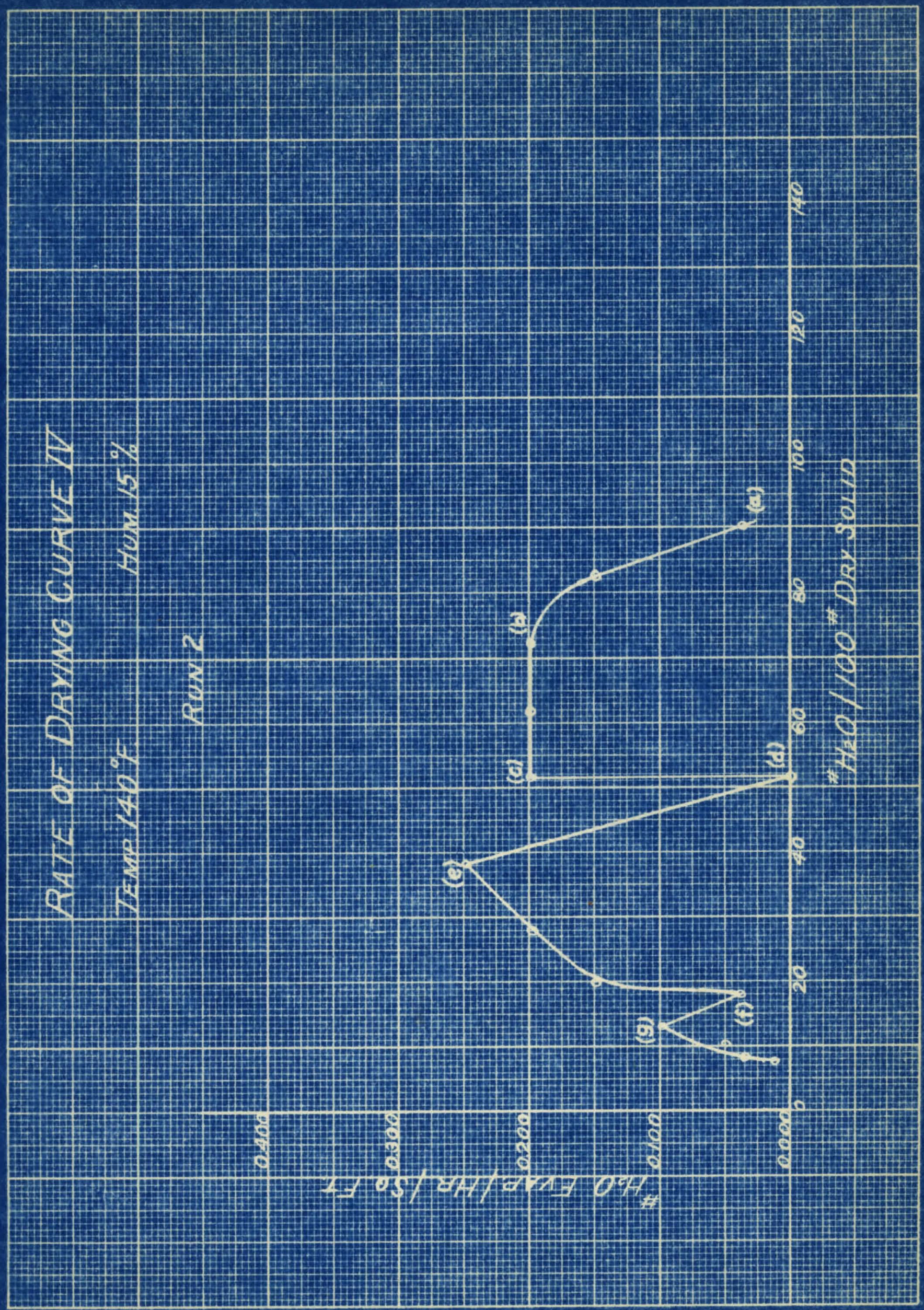


The dry Ing condit1one for Run 2 wore a constant temperature of $240^{\circ} \mathrm{F}$, and a maldity of 16\%. Tho drying alr was $65 \%$ roclrouleted and mixed with stean to the desired muidity.

\section{Drying Carve III Indlcates a stouds} decrease in the molature content during the firat two hours. In the next two hours the $20 \times 1$ is neglig1ble, and the following alx hours whow ateady deorease. The noxt two hours show a wlight dearease, after whloh the percent molature slopes off toward the end of the run.

Rate of Drying Curvo IV shows a rapid Increase (a to b), followed by a perlod of constent rate (b to $c$ ), arter which the rate drops to zero at d. It again incroases (d to o), followed by a ralling rate period (e to $f$ ). The rate of aryling increases ( $f$ to $g$ ), and then deoreasen towards the ond of the run. 
TABLE VIII

LOO SHEET

Fun 3

Dopertment of Chemicel Englnoering

Speed Selent1f10 School

Univeralty of Loulavilie

DRYINO OF PRUSSIAN BLUE

Paychrometer Chart No. 5

Date: $2 / 15 / 34$

Plant Record Data

Laboratory Data

Name: C. P. Blue

Rx. No.: 4012

SIIp Ho.: 674

Bat oh No.: $A-1$

Deto struck: $2 / 13 / 34$

No. Tray Dried: 20

Date In:

$2 / 15 / 34$

Batch Ficd :

$2 / 14 / 34$

Baton Rtd:; $2 / 21 / 34$

Drying Conditions

Temperature: $180^{\circ} \mathrm{F}$. Humid1ty: is\%

Inlet: Average Dry Bulb Temperature: $78^{\circ} \mathrm{F}$.
Average Wot Bulb Tomperature: ${ }^{\circ}{ }_{\mathrm{F}}^{\mathrm{F}}$.
Average Absolute Humldity: $83 \%$

Outlet: Average Dry Bulb Temperature: $151^{\circ} \mathrm{F}$. Average Wet Bulb Temperature: $130^{\circ} \mathrm{F}$. Average Absolute llomility: $48 \%$

Average Room Tomperature: $79^{\circ} \mathrm{F}$. Inlet Air Velocity: $108 \mathrm{rt} . / \mathrm{min}$. Percent Reclrculation! $93 \%$

Recorded Psychrometer Chart Temperatures: Dry Bulb: $180^{\circ} \mathrm{F}$. Wot Bulb: $126^{\circ} \mathrm{F}$,

Pan Data

\begin{tabular}{|c|c|c|c|}
\hline $\begin{array}{l}\text { Pan } \\
\text { No. }\end{array}$ & $\begin{array}{l}\text { Wt. of Cake } \\
\text { Before Dry ing } \\
\text { Ibs. }\end{array}$ & $\begin{array}{c}\text { Wt. of Cake } \\
\text { After Drying } \\
\text { ibs. }\end{array}$ & $\begin{array}{l}\text { Wt. Molsture } \\
\text { Lost } \\
\text { Ibs. }\end{array}$ \\
\hline $\begin{array}{l}1 \\
2 \\
3 \\
4 \\
5 \\
6\end{array}$ & $\begin{array}{r}47.75 \\
51.00 \\
48.75 \\
50.25 \\
50.50 \\
46.75 \\
18 \quad \frac{45.00}{295}\end{array}$ & $\begin{array}{l}25.75 \\
25.00 \\
24.00 \\
24.00 \\
25.00 \\
22.50 \\
146.25\end{array}$ & $\begin{array}{l}22.00 \\
26.00 \\
24.75 \\
26.25 \\
25.50 \\
24.25 \\
148.15\end{array}$ \\
\hline
\end{tabular}


TABIE $\mathbf{X}$

HOISTURE COMTEWT DATA

Run 3

\begin{tabular}{|c|c|c|c|c|}
\hline $\begin{array}{c}\text { Sample } \\
\text { No. }\end{array}$ & Hoy & $\begin{array}{c}\text { Wt. Wet } \\
\text { Semple } \\
\text { gme. }\end{array}$ & $\begin{array}{c}\text { Molotware } \\
\text { Lest } \\
\text { ens: }\end{array}$ & $\begin{array}{l}\text { Peroent } \\
\text { Moleture }\end{array}$ \\
\hline 1 & $\begin{array}{l}1 \\
2 \\
3 \\
4 \\
6 \\
6\end{array}$ & $\begin{array}{l}6.060 \\
6.009 \\
6.172 \\
6.170 \\
6.285 \\
5.416\end{array}$ & $\begin{array}{l}3.026 \\
3.284 \\
2.858 \\
3.896 \\
5.200 \\
2.884 \\
\text { Arexase }\end{array}$ & $\begin{array}{l}49.8 \\
54.1 \\
56.2 \\
55.4 \\
62.6 \\
58.5 \\
68.6\end{array}$ \\
\hline 8 & $\begin{array}{l}2 \\
2 \\
5 \\
8 \\
6 \\
6\end{array}$ & $\begin{array}{l}5.897 \\
5.797 \\
4.991 \\
5.991 \\
5.851 \\
5.156\end{array}$ & $\begin{array}{l}2.882 \\
2.982 \\
2.724 \\
3.294 \\
3.089 \\
2.717 \\
\text { A. }\end{array}$ & $\begin{array}{l}48.4 \\
51.4 \\
54.6 \\
55.8 \\
51.9 \\
58.7 \\
62.0\end{array}$ \\
\hline 3 & $\begin{array}{l}2 \\
8 \\
8 \\
4 \\
6 \\
6\end{array}$ & $\begin{array}{l}5.652 \\
5.894 \\
4.838 \\
5.896 \\
5.928 \\
4.300\end{array}$ & $\begin{array}{l}2.721 \\
3.019 \\
2.658 \\
3.087 \\
3.079 \\
8.152 \\
\text { Average }\end{array}$ & $\begin{array}{l}46.5 \\
51.1 \\
53.4 \\
51.3 \\
51.2 \\
49.1 \\
50.6\end{array}$ \\
\hline 4 & $\begin{array}{l}1 \\
8 \\
5 \\
8 \\
5 \\
6\end{array}$ & $\begin{array}{l}5.784 \\
5.288 \\
4.621 \\
5.431 \\
5.644 \\
4.670\end{array}$ & $\begin{array}{l}2.896 \\
2.828 \\
2.345 \\
2.617 \\
2.745 \\
2.104 \\
\text { Arewago }\end{array}$ & $\begin{array}{l}44.8 \\
47.8 \\
58.1 \\
48.8 \\
48.7 \\
45.1 \\
44.8\end{array}$ \\
\hline 5 & $\begin{array}{l}1 \\
2 \\
5 \\
4 \\
5 \\
6\end{array}$ & $\begin{array}{l}5.449 \\
5.489 \\
4.986 \\
5.886 \\
5.848 \\
4.986\end{array}$ & $\begin{array}{l}2.094 \\
2.520 \\
2.497 \\
2.506 \\
2.656 \\
1.979\end{array}$ & $\begin{array}{l}38.4 \\
46.0 \\
50.0 \\
43.2 \\
45.4 \\
39.7\end{array}$ \\
\hline
\end{tabular}


TABIS IX (cont.)

MOISTURE CONTEIT DATA

$\operatorname{Tun} 3$

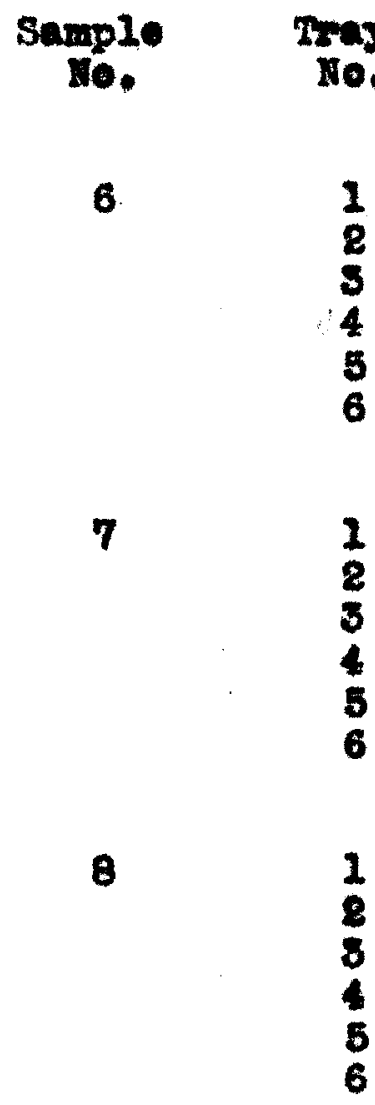

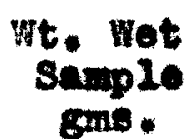

5.250
5.306
4.348
5.800
5.686
5.484

7.185

5.308

4.892

8.919

5.028

7.117

9

20

1
8
8
4
5
6

1
8
8
5
6

9.022

6.698

6.826

6.505

6.719

5.305

6.975

5.982

6.578

5.488

5.842

4.538

1
2
3
4
6
6
Vo1sture

Lost

gens.

2.016

2.275

2.024

2.018

2.441

1.129

A rexase

$1: 932$

2.027

1.877

2. 608

1.942

2.135

Arerage

2.327

2.465

2.453

1.658

2.658

0.763

A 7 rage

1. 779

1.861

2.627

2.084

1.761

0.589

Average

1. 874

1.016

1.655

0.843

1.306

0.779

Averago
Poroent

noleture

38.4
48.8
46.7
38.8
44.8
32.6
40.6

27.9

38.0

48.7

29.2

38.7

$\frac{89.7}{3.5}$

25.8

36.8

37.7

28.0

38.8

14.4

30.0

85. 5

21.2

40.0

18. 1

30.9

11.9

27.6

$29 \cdot 4$

18.6

25.0

14.0

18.6

14.4 


\author{
MaLE IX (Cont.) \\ MOISTURE CONTEHT DATA
}

Fun 3

\begin{tabular}{|c|c|c|c|c|}
\hline $\begin{array}{c}\text { Semple } \\
110 .\end{array}$ & $\begin{array}{c}\text { Tray } \\
\text { No. }\end{array}$ & $\begin{array}{c}\text { Wt. wot } \\
\text { Sauple } \\
\text { gens. }\end{array}$ & $\begin{array}{c}\text { Modsture } \\
\text { Lost } \\
\text { gms. }\end{array}$ & $\begin{array}{l}\text { Pereont } \\
\text { Moligture }\end{array}$ \\
\hline 21 & $\begin{array}{l}1 \\
2 \\
3 \\
4 \\
5 \\
6\end{array}$ & $\begin{array}{l}5.358 \\
6.175 \\
4.708 \\
5.051 \\
5.207 \\
4.561\end{array}$ & $\begin{array}{l}0.599 \\
0.653 \\
0.675 \\
0.561 \\
0.641 \\
0.148 \\
\text { Average }\end{array}$ & $\begin{array}{r}21.8 \\
10.8 \\
14.8 \\
10.8 \\
12.8 \\
3.8 \\
10.4\end{array}$ \\
\hline 18 & $\begin{array}{l}1 \\
2 \\
3 \\
4 \\
5 \\
6\end{array}$ & $\begin{array}{c}3.248 \\
6.818 \\
5.675 \\
6.198 \\
5.684 \\
5.279 \\
.\end{array}$ & $\begin{array}{l}0.089 \\
1.208 \\
1.129 \\
0.717 \\
0.083 \\
0.801 \\
\text { A vernge }\end{array}$ & $\begin{array}{r}2.8 \\
\times 19.1 \\
\times 19.7 \\
12.7 \\
5.9 \\
3.8 \\
6.1\end{array}$ \\
\hline 13 & $\begin{array}{l}1 \\
8 \\
8 \\
4 \\
5 \\
6\end{array}$ & $\begin{array}{l}5.186 \\
6.238 \\
5.028 \\
6.657 \\
7.099 \\
4.878\end{array}$ & $\begin{array}{l}0.440 \\
0.850 \\
0.348 \\
0.170 \\
0.728 \\
0.161 \\
\text { Average }\end{array}$ & $\begin{array}{r}8.5 \\
9.2 \\
5.8 \\
7.1 \\
\times 10.8 \\
3.3 \\
6.3\end{array}$ \\
\hline 14 & $\begin{array}{l}1 \\
2 \\
8 \\
4 \\
5 \\
6\end{array}$ & $\begin{array}{l}5.018 \\
7.055 \\
4.165 \\
5.916 \\
5.765 \\
5.080\end{array}$ & $\begin{array}{l}0.108 \\
0.280 \\
0.078 \\
0.188 \\
0.347 \\
0.154 \\
\text { Averege }\end{array}$ & $\begin{array}{l}2.8 \\
1.8 \\
1.8 \\
2.7 \\
6.0 \\
3.0 \\
2.0\end{array}$ \\
\hline is & $\begin{array}{l}1 \\
8 \\
3 \\
4 \\
5 \\
6\end{array}$ & $\begin{array}{l}3.247 \\
5.958 \\
4.486 \\
4.500 \\
4.776 \\
4.920\end{array}$ & $\begin{array}{l}0.034 \\
0.068 \\
0.058 \\
0.081 \\
0.118 \\
0.122 \\
\text { Average }\end{array}$ & $\begin{array}{l}1.1 \\
1.0 \\
1.3 \\
1.8 \\
2.5 \\
2.5 \\
1.7\end{array}$ \\
\hline
\end{tabular}

$x$ Value cultted in avorago. 


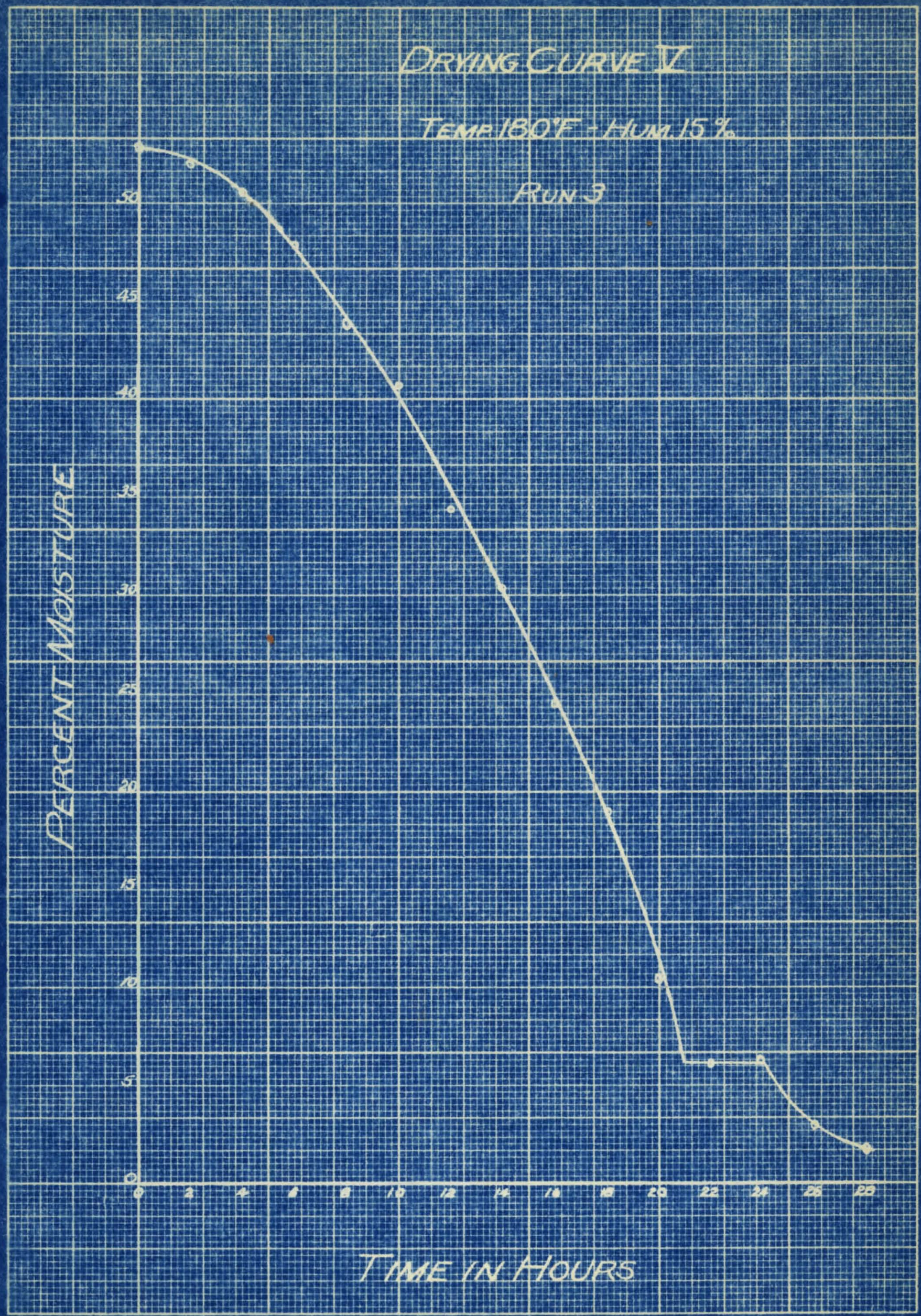


MATE $\mathbf{x}$

RATS OP DATMO DAT

$\operatorname{Ran} 5$






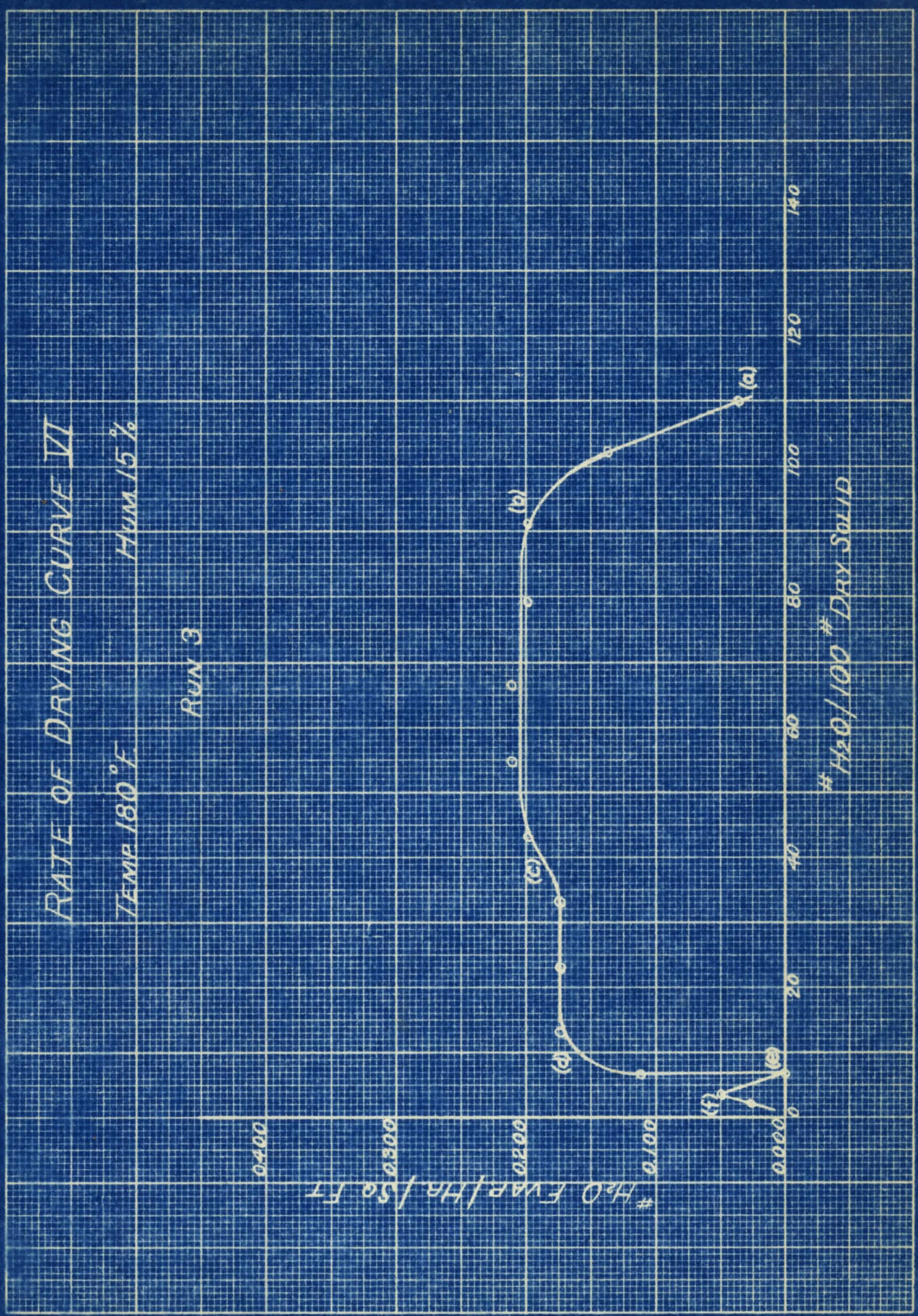


The dryins conditions for Run 3 wore constant temperature of $180^{\circ} \mathrm{g}$., and a constant monldity of 25\%. Tho drying alr was 056 reolroulated, and mixed with steam to the dosired conditions.

Drying Curvo $v$ shows ateeds decrease In the percent molsture during the firet twentyon houre. For the next three hours the lose in no1ature 18 practicalig soro, after which the percent molature decreaces till the and of the ran.

Rate of Dirging Curve VI Indieates an Increace in the rate of drying (a to b). Tho rate thon remelns oonstant from b to d, except for a elight dip at 0 . Woxt the rate of drying deoreaser abruply (a to 0 ), egaln inoreases (o to $f$ ), and then decrenses towards the end of the man. 


\section{TABLE XI}

\section{I0O SHEET}

Run 4

Departmont of Chemlonl Bnglneoring

speed solentirie school

Univeralts of Loulavilie

\section{DRYINO OF PFUSSIAX BLOE}

Peychromoter Chart No. 4

Plant Rocord Data

Hame: C. P. Blue

Rx, No.: 4012

SIIp No. 647

Batoh No.: 3

Date struok: $2 / 14 / 34$

Ho. Traye Dried: 20

Dato In: $2 / 21 / 34$
Date: 2/22/34

Laboratory Data

Bat oh rod.: 2/21/34

Bateh Rtd.: $2 / 28 / 34$

Drying Condition

Temperatures $180^{\circ} \mathrm{F}$.

Aunidity! $80 \%$

Inlet: Average Dry Bulb Tomperature: $\quad 75^{\circ} \mathrm{F}$. Average Wot Bulb Tomperature: Outlet: Avorage Dary Bulb Temperature: $151^{\circ} \mathrm{P}$. Average Absolute Hulatty $58 \%$

Average Room Tomporature: $76^{\circ} \mathrm{F}$.

Inlot A1r Veloc1ty: $170 \mathrm{ft.} / \mathrm{mln} /$

Percent Rocirculation: O4\%

Recorded Peyohroneter Chart "omperatures, Dry Bulb: $180^{\circ} \mathrm{F}$. Wot BuIb: $237^{\circ} \mathrm{F}$.

Pan Data

Pan Wt, of Cake
Ho. Before Drying
ibs.

$$
\begin{aligned}
& \text { Wt. of Cake } \\
& \text { Arter Drying } \\
& \text { Ibs. } \\
& 25.00 \\
& 23.80 \\
& 23.80 \\
& 22.50 \\
& 85.00 \\
& 23.80 \\
& 143.00
\end{aligned}
$$$$
\text { Aftor Drying }
$$$$
\text { Wt. Molsture }
$$$$
\text { Loat }
$$$$
\text { Ibe. }
$$

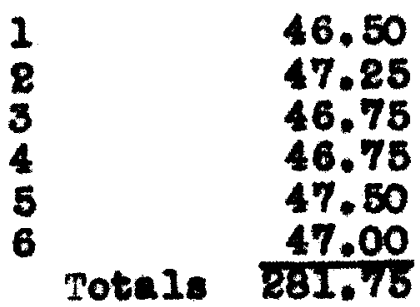

21.50

23.75

23.25

24.26

22.50

23.50

ISB.15 
MBIE XII

YOISIURE COWMEN DATA

Pran 4

\begin{tabular}{|c|c|c|c|c|}
\hline $\begin{array}{l}\text { Sump } 20 \\
\text { to. }\end{array}$ & $\begin{array}{l}\text { Trovy } \\
\text { No. }\end{array}$ & $\begin{array}{c}\text { Wt Wet } \\
\text { 3ample } \\
\text { gans }\end{array}$ & $\begin{array}{c}\text { Holdoure } \\
\text { toat } \\
\text { gms. }\end{array}$ & $\begin{array}{l}\text { Perent } \\
\text { Moletrut }\end{array}$ \\
\hline 1 & $\begin{array}{l}1 \\
2 \\
5 \\
4 \\
6 \\
6\end{array}$ & $\begin{array}{l}6.089 \\
5.802 \\
6.025 \\
6.187 \\
5.768 \\
6.085\end{array}$ & $\begin{array}{l}3.506 \\
3.597 \\
3.444 \\
3.658 \\
5.404 \\
3.548 \\
\text { Avarag }\end{array}$ & $\begin{array}{l}58.0 \\
60.9 \\
57.3 \\
88.8 \\
59.1 \\
58.2 \\
68.7\end{array}$ \\
\hline $\mathbf{2}$ & $\begin{array}{l}1 \\
2 \\
6 \\
4 \\
5 \\
6\end{array}$ & $\begin{array}{l}5.582 \\
5.581 \\
5.580 \\
5.607 \\
5.617 \\
5.899\end{array}$ & $\begin{array}{l}3.226 \\
3.389 \\
3.157 \\
3.190 \\
3.869 \\
3.412 \\
\text { Arexago }\end{array}$ & $\begin{array}{l}57.5 \\
69.8 \\
57.2 \\
57.0 \\
59.3 \\
57.8 \\
58.1\end{array}$ \\
\hline 8 & $\begin{array}{l}1 \\
2 \\
5 \\
4 \\
5 \\
6\end{array}$ & $\begin{array}{l}5.881 \\
5.889 \\
5.461 \\
4.882 \\
5.485 \\
5.558\end{array}$ & $\begin{array}{l}3.060 \\
2.255 \\
5.076 \\
2.747 \\
3.151 \\
3.125 \\
\text { Av02080 }\end{array}$ & $\begin{array}{l}85.8 \\
57.5 \\
56.8 \\
56.8 \\
57.4 \\
56.6 \\
56.6\end{array}$ \\
\hline 4 & $\begin{array}{l}1 \\
2 \\
3 \\
4 \\
6 \\
6\end{array}$ & $\begin{array}{r}5.584 \\
6.283 \\
5.119 \\
5.408 \\
5.809 \\
\text { 5.392 }\end{array}$ &  & $\begin{array}{l}54.6 \\
66.8 \\
55.3 \\
58.9 \\
58.0 \\
54.5 \\
65.6\end{array}$ \\
\hline $\mathbf{5}$ & $\begin{array}{l}1 \\
2 \\
3 \\
4 \\
5 \\
6\end{array}$ & $\begin{array}{l}5.002 \\
5.807 \\
5.101 \\
4.687 \\
5.570 \\
5.009\end{array}$ & $\begin{array}{l}2.644 \\
2.980 \\
2.758 \\
2.451 \\
3.162 \\
2.688\end{array}$ & $\begin{array}{l}52.8 \\
55.4 \\
55.6 \\
51.8 \\
56.7 \\
53.0\end{array}$ \\
\hline
\end{tabular}


TABLE XII (Cont.)

MoIsTure CONTBNT DATA

Run 4

\begin{tabular}{|c|c|c|c|c|}
\hline $\begin{array}{c}\text { Semplo } \\
\text { no. }\end{array}$ & $\begin{array}{c}\text { Tray } \\
\text { No. }\end{array}$ & $\begin{array}{c}\text { Wt. Wet } \\
\text { semplo } \\
\text { gms. }\end{array}$ & $\begin{array}{l}\text { Mol ture } \\
\text { Lost } \\
\text { gans. }\end{array}$ & $\begin{array}{l}\text { Percont } \\
\text { Molature }\end{array}$ \\
\hline 6 & $\begin{array}{l}1 \\
2 \\
3 \\
4 \\
5 \\
6\end{array}$ & $\begin{array}{l}4.645 \\
5.260 \\
5.075 \\
4.895 \\
5.898 \\
4.646\end{array}$ & $\begin{array}{l}2.362 \\
2.737 \\
2.689 \\
2.867 \\
2.908 \\
2.438 \\
\text { Average }\end{array}$ & $\begin{array}{l}50.8 \\
51.9 \\
51.9 \\
51.8 \\
56.4 \\
58.4 \\
82.4\end{array}$ \\
\hline 7 & $\begin{array}{l}1 \\
2 \\
3 \\
4 \\
5 \\
6\end{array}$ & $\begin{array}{l}5.206 \\
5.188 \\
4.018 \\
5.079 \\
5.856 \\
4.685\end{array}$ & $\begin{array}{l}2.575 \\
2.715 \\
2.496 \\
2.481 \\
2.892 \\
2.234 \\
\text { A.7019.8 }\end{array}$ & $\begin{array}{l}49.3 \\
58.3 \\
80.8 \\
47.7 \\
54.8 \\
48.3 \\
51.0\end{array}$ \\
\hline 8 & $\begin{array}{l}1 \\
2 \\
5 \\
4 \\
5 \\
6\end{array}$ & $\begin{array}{l}4.410 \\
4.877 \\
4.819 \\
4.441 \\
4.851 \\
4.477\end{array}$ & $\begin{array}{l}2.051 \\
2.129 \\
2.378 \\
2.137 \\
2.628 \\
2.178 \\
\text { A. }\end{array}$ & $\begin{array}{l}46.6 \\
49.8 \\
49.8 \\
46.0 \\
54.2 \\
48.6 \\
49.4\end{array}$ \\
\hline 8 & $\begin{array}{l}1 \\
2 \\
3 \\
4 \\
5 \\
6\end{array}$ & $\begin{array}{l}4.586 \\
5.044 \\
4.386 \\
4.347 \\
4.963 \\
4.568\end{array}$ & $\begin{array}{l}2.094 \\
2.223 \\
1.255 \\
1.743 \\
2.565 \\
2.088 \\
\text { Average }\end{array}$ & $\begin{array}{l}45.7 \\
44.8 \\
44.4 \\
40.1 \\
51.5 \\
45.8 \\
45.5\end{array}$ \\
\hline 10 & $\begin{array}{l}1 \\
2 \\
3 \\
4 \\
5 \\
6\end{array}$ & $\begin{array}{l}4.740 \\
4.879 \\
4.758 \\
3.796 \\
4.760 \\
4.608\end{array}$ & $\begin{array}{r}1.994 \\
1.885 \\
2.285 \\
1.828 \\
2.325 \\
1.798 \\
\text { Average }\end{array}$ & $\begin{array}{l}42.0 \\
38.0 \\
44.8 \\
38.2 \\
48.2 \\
38.0 \\
40.8\end{array}$ \\
\hline
\end{tabular}




\section{mABLE XII (Cont.) \\ MOISTURE CONTEMT DM}

Pan 4

\begin{tabular}{|c|c|c|c|c|}
\hline $\begin{array}{c}\text { Samplo } \\
\text { no. }\end{array}$ & $\begin{array}{l}\text { Tray } \\
\text { Bo. }\end{array}$ & $\begin{array}{c}\text { wt. net } \\
\text { simplo } \\
\text { gens. }\end{array}$ & $\begin{array}{l}\text { Holsture } \\
\text { Lost } \\
\text { gent. }\end{array}$ & $\begin{array}{l}\text { Porcent } \\
\text { Molotive }\end{array}$ \\
\hline 22 & $\begin{array}{l}1 \\
8 \\
3 \\
4 \\
5 \\
6\end{array}$ & $\begin{array}{r}4.822 \\
3.826 \\
8.688 \\
4.758 \\
4.529 \\
4.590\end{array}$ & $\begin{array}{l}1.846 \\
1.297 \\
1.377 \\
0.858 \\
2.176 \\
2.174 \\
\text { Averngo }\end{array}$ & $\begin{array}{r}38.8 \\
33.8 \\
37.6 \\
18.0 \\
50.2 \\
\times 47.3 \\
36.6\end{array}$ \\
\hline 28 & $\begin{array}{l}1 \\
2 \\
3 \\
4 \\
5 \\
6\end{array}$ & $\begin{array}{l}4.318 \\
4.451 \\
5.165 \\
6.056 \\
3.606 \\
4.145\end{array}$ & $\begin{array}{l}1.586 \\
1.514 \\
1.585 \\
1.360 \\
1.745 \\
1.468 \\
\text { A verago }\end{array}$ & $\begin{array}{r}36.0 \\
34.0 \\
29.7 \\
\times 26.8 \\
48.4 \\
35.4 \\
36.7\end{array}$ \\
\hline 13 & $\begin{array}{l}1 \\
2 \\
3 \\
4 \\
5 \\
6\end{array}$ & $\begin{array}{l}5.468 \\
6.678 \\
6.548 \\
5.464 \\
5.911 \\
6.698\end{array}$ & $\begin{array}{l}1.088 \\
1.508 \\
1.700 \\
0.508 \\
2.828 \\
1.118 \\
\text { Avarage }\end{array}$ & $\begin{array}{r}19.8 \\
23.9 \\
86.6 \\
9.8 \\
37.7 \\
16.7 \\
28.5\end{array}$ \\
\hline 14 & $\begin{array}{l}1 \\
2 \\
3 \\
4 \\
5 \\
6\end{array}$ & $\begin{array}{l}7.121 \\
5.724 \\
6.321 \\
6.081 \\
7.145 \\
5.925\end{array}$ & $\begin{array}{l}1.569 \\
0.598 \\
0.708 \\
0.834 \\
1.861 \\
0.800 \\
\text { Aterage }\end{array}$ & $\begin{array}{r}22.1 \\
10.3 \\
11.5 \\
8.3 \\
26.0 \\
13.5 \\
13.8\end{array}$ \\
\hline 15 & $\begin{array}{l}1 \\
8 \\
5 \\
4 \\
5 \\
6\end{array}$ & $\begin{array}{l}4.838 \\
4.454 \\
3.278 \\
4.624 \\
6.829 \\
5.587\end{array}$ & $\begin{array}{l}0.218 \\
0.438 \\
0.128 \\
0.214 \\
1.647 \\
0.808 \\
\text { Average }\end{array}$ & $\begin{array}{r}4.4 \\
9.8 \\
4.7 \\
4.6 \\
26.5 \\
5.8 \\
9.8\end{array}$ \\
\hline
\end{tabular}

$x$ Value onltted in average. 


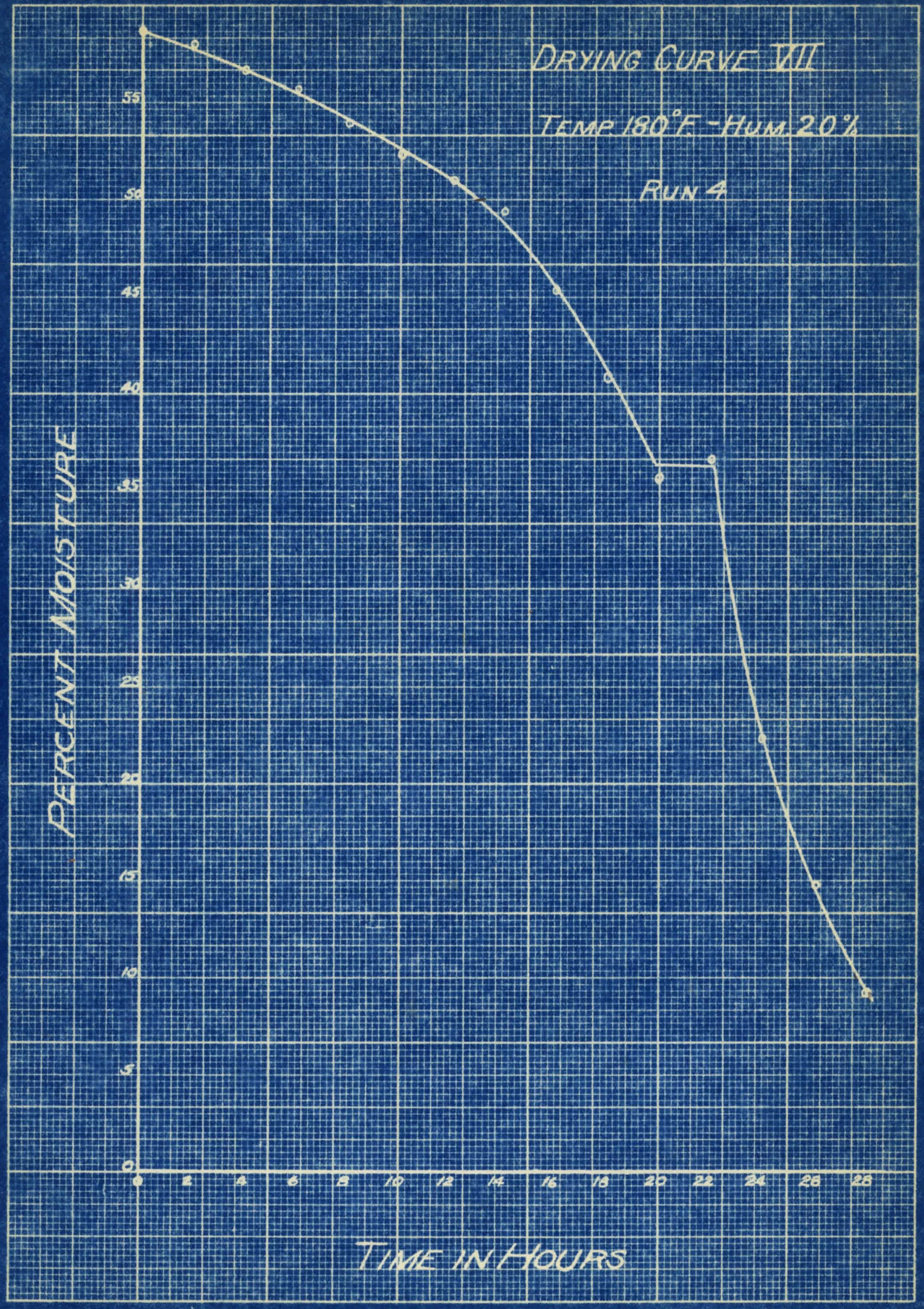


TABLE XIII

RATE OF DAIINO DATA

Fun 4

\begin{tabular}{|c|c|c|c|c|c|c|c|}
\hline $\begin{array}{c}\text { sample } \\
\text { Ho. }\end{array}$ & $\begin{array}{c}\text { Percont } \\
\text { Moletwre } \\
\text { (From Curve) }\end{array}$ & $\begin{array}{l}\text { Wt. Wot } \\
\text { Cake } \\
\text { 1bes. }\end{array}$ & $\begin{array}{c}\text { Wt. } \mathrm{D}_{2 y} \\
\text { Culro } \\
\text { 2bin. }\end{array}$ & $\begin{array}{l}\text { Yolature } \\
\text { Prosent } \\
\text { ibe. }\end{array}$ & $\begin{array}{l}\text { Molature } \\
\text { Iost } \\
\text { Ibe. }\end{array}$ & 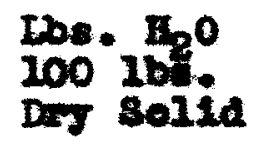 & 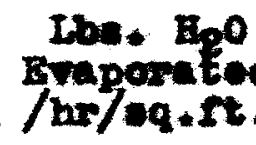 \\
\hline $\begin{array}{r}1 \\
8 \\
8 \\
4 \\
5 \\
6 \\
7 \\
8 \\
9 \\
10 \\
11 \\
12 \\
13 \\
14 \\
15\end{array}$ & $\begin{array}{r}58.7 \\
57.7 \\
56.6 \\
55.5 \\
54.1 \\
52.6 \\
57.0 \\
48.7 \\
45.5 \\
42.1 \\
36.4 \\
36.3 \\
22.3 \\
14.5 \\
9.4\end{array}$ & $\begin{array}{l}282 \\
276 \\
268 \\
262 \\
254 \\
246 \\
238 \\
227 \\
814 \\
108 \\
184 \\
183 \\
160 \\
156 \\
129\end{array}$ & $\begin{array}{l}116 \\
116 \\
116 \\
116 \\
116 \\
116 \\
116 \\
116 \\
116 \\
116 \\
116 \\
116 \\
116 \\
117 \\
116\end{array}$ & $\begin{array}{r}166 \\
160 \\
152 \\
146 \\
138 \\
180 \\
128 \\
111 \\
90 \\
88 \\
68 \\
67 \\
34 \\
80 \\
18\end{array}$ & $\begin{array}{r}- \\
6 \\
8 \\
6 \\
6 \\
8 \\
8 \\
11 \\
13 \\
16 \\
14 \\
1 \\
38 \\
14 \\
7\end{array}$ & $\begin{array}{r}143.4 \\
138.0 \\
131.0 \\
126.0 \\
110.0 \\
112.0 \\
105.0 \\
95.7 \\
84.5 \\
70.7 \\
58.6 \\
57.7 \\
29.5 \\
17.8 \\
11.8\end{array}$ & $\begin{array}{l}-5-1 \\
0.074 \\
0.009 \\
0.074 \\
0.009 \\
0.009 \\
0.090 \\
0.187 \\
0.162 \\
0.198 \\
0.174 \\
0.018 \\
0.109 \\
0.174\end{array}$ \\
\hline
\end{tabular}




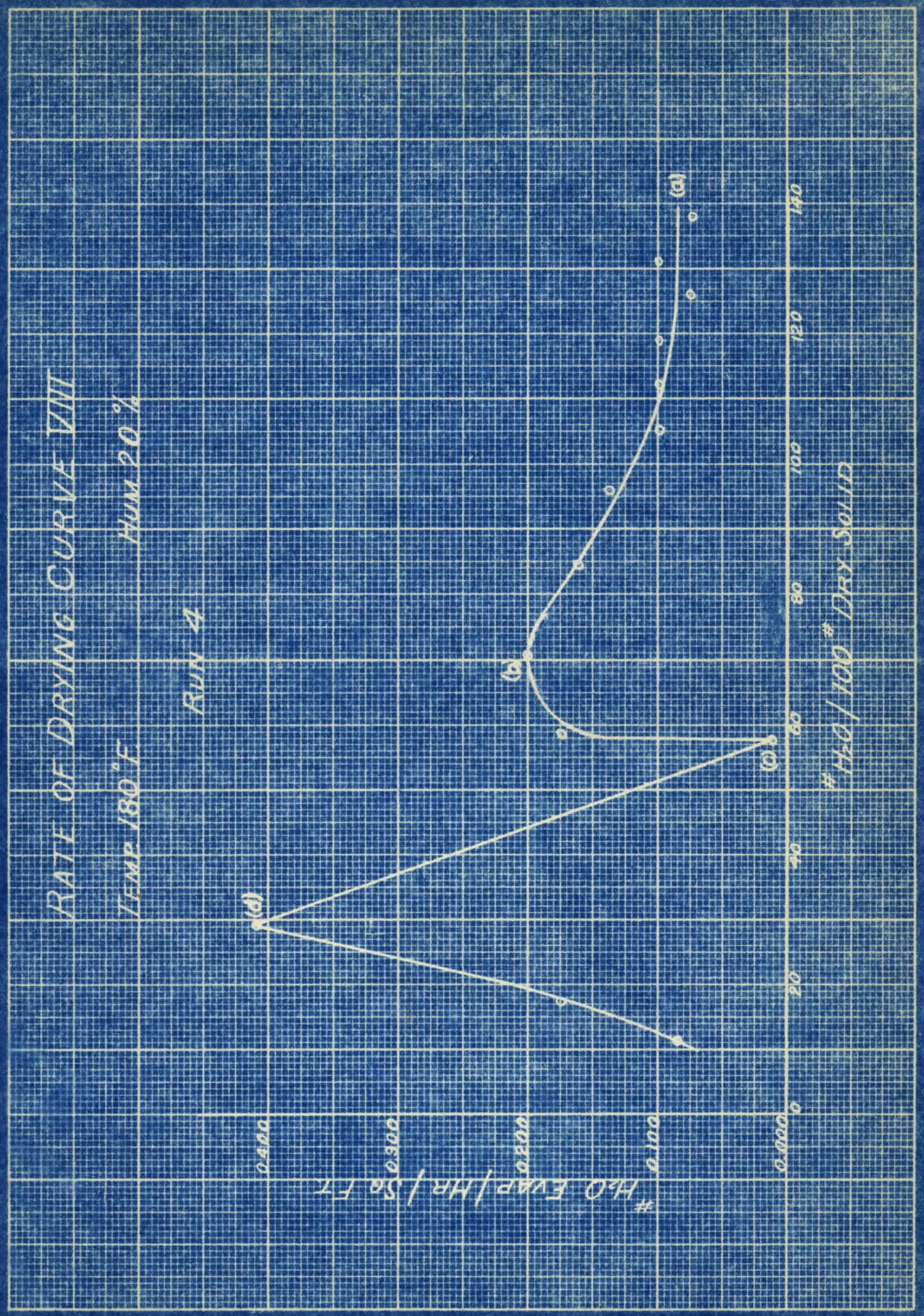


Dy Ing Condition for Run 4 were a constant temperature of $180^{\circ} \mathrm{F}$, , and a bumlalty of 20\%. The drying air was $94 \%$ roolroulated and mixed with stean to the desired oonditions.

Drying curro VII Indicates an IncreasIng lose in molsture during the first twenty hours. For the next two houre the $100 \mathrm{~s}$ is prectically zero, after which the lose is rery rapid until the end of the ran.

The Rate of Drying Curve VIII how: -lowly increasing rate (a to b). The rate noxt epproaches zero (b to 0 ), beomes very hlgh (o to d), and then decreasen rapldy unt1l the end of the run. 
strun 
In enwarising the work on the alr drying of Pruselan Blue, it is well to inolude all of the work done, both in this investigation and in that performed by Sohmied and Ardern (1). The curves (drying and rate of drying) resulting from these teata have imilar characterietica with those of the previous work.

\section{All of the teats give a drying curve} which atarts at appraximetely fifty percent molsm ture content, and drops off with a fulrig otends lope for several hours. About midway on each curve there appears "break", period whon the slope of the curve changes oonelderably, in some cases oven becoming zero. After this "break" the drying contimes ateadily unt1l the curve bogine to level out parallel to the Timo-axis.

The rate of arying curves are 100 imllar to each otber in character. Daring the oaris part of each ran the curve shows an lncressing or steady rate of drying. Corresponaing to the "breake" on the arylng curven, the curves of rate of drying show an abrupt "dip", when for period the drying rate 1s vory low, or zero. After the 
"dip" the rate of arging again becomes high, but docreases rapldiy tomards zero.

The rate of drying ourves mey be divided Into two parts in the same manor as the theoretical drying rate curres. In Fig. 4 a typical rate curve of this investigation is compared with tho theoretical curve of Newran (2). The rirat part of each of those curves corresponde to the theoretical constant rete period. During this part of the dryling the cake is fairly soft, and ovaporation of molature the exposed surface of the cake may be considered as the controlling feotor in the drying.

Thet part of tho drying curvo containing and following the "alp" is equivalent to the theorotical falling rate period. The lant portion of each curve following the dip, when the rate of arying is rapidiy decreasing, is the sone whore internal difruaion controls. During this part of the run the cake is $\mathrm{dry}$, hard, and Increasingly brittio, with a tendenoy to crumble.

The rirst and last portion of the Prusalan Blue drying rate curres correspond well with the firat period and the second sone of the second 


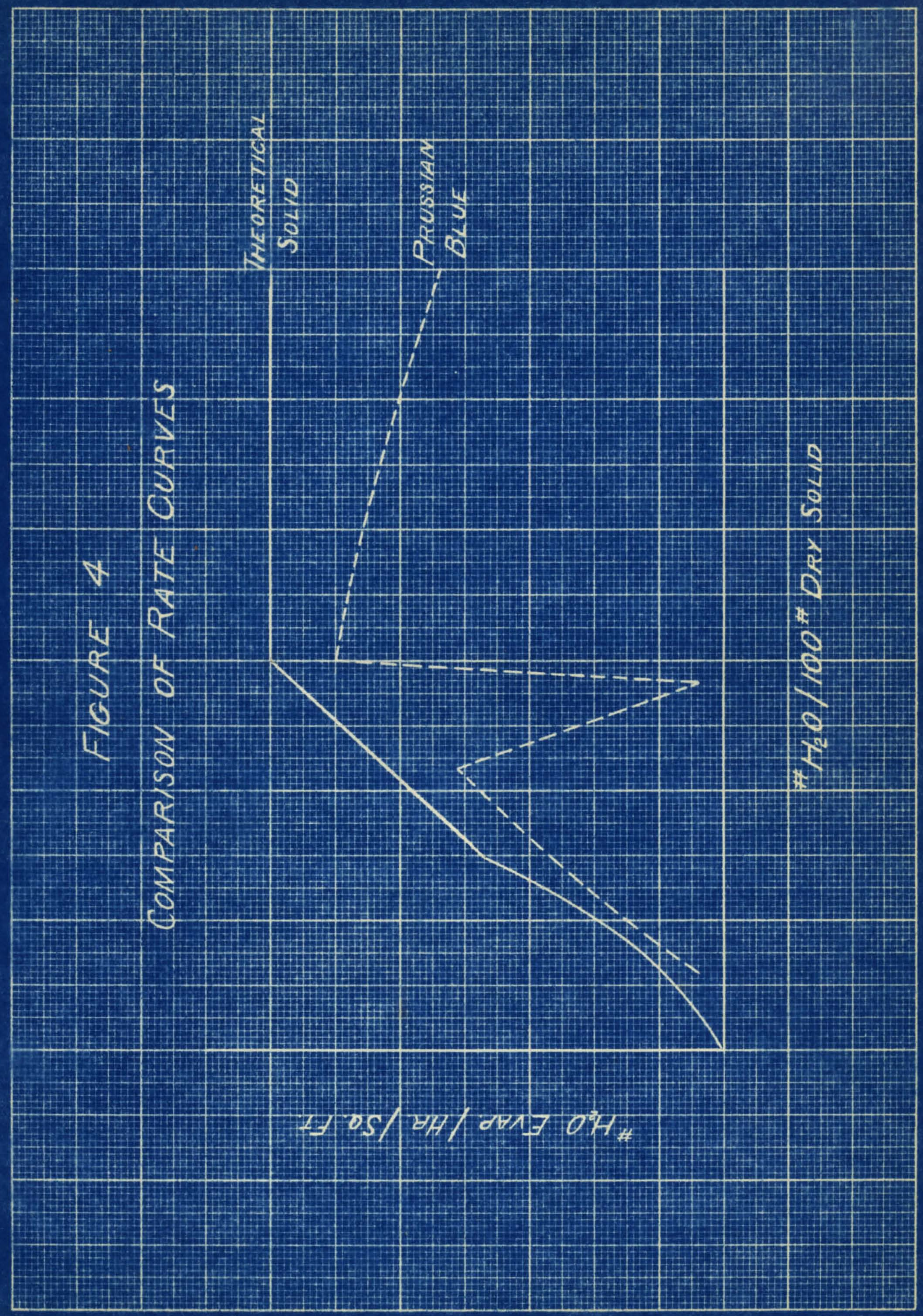


perlod of the thooretical curve. However, the Intermediate "dips" of the Prusaian Blue ourves are not as oally correlated with the theoretical sone of unseturated ourface drying. Tho question eppears to be to explain these "dips".

It hal been observed that tho "dip" of the drying rate curve correaponds to the "break" on the drying curre. A comparimon of the varlous drying ourves is more easily obteined than of the alfferent rate of arying curves. In order to obtaln such a comparison, Tuble XIV has beon propared from the different drying curves of this investigation and of the previous work (1). It w121 be noticed that fruns 8 and 2-A seoh show two "breake".

It 1s alfricult to find any consistancy about the molature content at whioh "broaks" occur. It varies from ix to thirty-e1x percent, the average being a 12tt1e over twenty-two percent. Thore appeare to be no correlation between temperature or muldity and the molsture oontent at the "broak". Thorefore, it may be concluded thet tho particular drying conditions have little to do with the occurrence of these"breaka". 
COMPARISON OF AIR DRYINO CURNES

\begin{tabular}{|c|c|c|c|c|c|c|}
\hline $\begin{array}{l}\text { Run } \\
\text { No. }\end{array}$ & Tgmp. & $\begin{array}{l}\text { mund- } \\
\text { a1ty }\end{array}$ & $\begin{array}{l}\text { Trme } \\
\text { Hre. }\end{array}$ & $\begin{array}{c}\text { Indtial } \\
\text { Moleture } \\
\text { Contont } \\
8\end{array}$ & $\begin{array}{c}\text { A vg. Molat. } \\
\text { Content } \\
\text { at Broak } \\
\$\end{array}$ & $\begin{array}{c}\text { Final } \\
\text { Molsture } \\
\text { Content } \\
\$\end{array}$ \\
\hline 1 & 180 & 6.3 & 28 & 49.0 & 34.0 & 23.0 \\
\hline 2 & 140 & 4.6 & 88 & 48.4 & 27.4 & 7.6 \\
\hline 5 & 140 & 10 & 28 & 47.8 & 34.9 & 8.7 \\
\hline 8 & 140 & 25 & 88 & 47.0 & $28.2 * 21.0$ & 10.8 \\
\hline R-A & 140 & 15 & 28 & 48.0 & $33.7 \div 15.8$ & 7.3 \\
\hline 8 & 160 & 6.3 & 28 & 48.0 & 28.3 & 8.8 \\
\hline 6 & 160 & 10 & 28 & 43.3 & 21.9 & 2.4 \\
\hline 9 & 160 & 25 & 28 & 46.4 & 22.5 & 8.7 \\
\hline 20 & 160 & $\infty$ & 28 & 46.2 & 20.3 & 16.0 \\
\hline 4 & 180 & 2.7 & 20 & 51.6 & 20.4 & 16.6 \\
\hline $1-A$ & 180 & 2.0 & 28 & 53.6 & 20.4 & 1.4 \\
\hline 7 & 280 & 20 & 88 & 43.4 & 26.0 & 7.8 \\
\hline $3-A$ & 180 & 26 & 28 & 58.5 & 6.8 & 1.6 \\
\hline $4-A$ & 180 & 20 & 28 & 58.7 & 36.4 & 9.3 \\
\hline
\end{tabular}

Fun Hos, 1-A, 2-A, 3-A, and 4-A from this Investigation. othor data from rork of sohmled and Ardorn (1). 
Tho possibility that those "breake" might signify thermal decomponition of the Blue prosented 1teelf. The products or such a docompo-1tion nould be hydrogen cyanide, cyanogen and ammonla gasen. Qualitative teste for these gases wore made on the outlet a1r, and they were found prosent in mime quantities only in the rery carllest part of tho convtant rate period.

It 1s auggested that the "broak" is cauned by a phrelonl change rather than a ohomlcal one. The constant rate period lasts unt1l the renidual froe moleture in the ploment is removed. Thon the arying becomes rery el1ght, or conses ent1rels. whilo the material undergoes a rearrangement of physical structure, with the resultant ilberation of more or los water of crystallization.

Romoval of this nowly liberated moleture causes an inorease in the rate of drying, but ac the exposed surface is quito dry, Internal resistance 1s the controlling factor in this drying. therefore the rate of drying deoreases an the resatance Increasea. 
The wide disorepancy in the occurence of the "break" Is belloved due ont1roly to the oboulanl proparation of the blue, and is not dependent upon the particular condition under whioh it is dried.

From Table XIV 1t w111 be observed that for ang particular temperature, the final moleture oontent incresees as the momidity increases. Also, for axp muldity, the sinal moisture content decreases as the temperature incrosece. These facte may bo explained from the fact thet the higher the humldity the higher the oquillbriun molsture content. But for an partioular muldity, the higher the ary bulb temperature, the greater the driving force between wet and ary bulb temperatures, and therefore the lower the rinal molsture content.

Rurther Investigations are now belng conducted in those laboratories with the view of determining, If possible, the exact character of the "breaks" and "dips" of the dryling and rete or aryIng curves in this study.

One series of teste are being conducted in the dryer, eimilar to the rune previously 
discussed. In these runi thermocouples are pleced above, below, and in rarione parte of the cake. The bypothesis is that the difference between the temperature of the cake 1 teelf and of the air abore the onke will depend upon the rate of drying. The actual cake temperature ahould be much more noarly equal to the control temperature at the bottom of the "dip", when the drying rate is noex soro, then on o1ther side of 1t, when the drying is taking plece more repldis. Simliarly, the cake approachos It equilibrium molsture content, tho drying rate diminishes, and there should bo corresponding rise In the cake temperature.

\section{There is no agreement emong previous} Investigators as to the amount of water of cryatallization in Prualan Blue. Since an explanation of the drying rate "dips" on the beris of tho breakIng oft of water of cryatallization bas beon offerod. It appeared desfrable to ondeavor to determine the exact water of cryatalizeation content of Prusalan Blue.

A serles of teats for this water of cryatallization are belng conductod by drying a 
omell quantity of Blue rapidiy by means of eleetrio hoat in an opon crucible. A thermoneter is placed In the conter of the oake, and the arucible ouspended on a balance, so as to read constently the temperature and the $108 \mathrm{~s}$ in weight of the cample.

Other teste for this water of crystall1sation are being conducted by mean of a olosed prossure bomb. A sample of wot plgment is sealod In the bomb, which is then Immersed in an oll bath and $20 \mathrm{w} 1 \mathrm{~J}$ heatod. Reading of both temperaturo and presaure are made at regular short intervals.

It 1o belloved that those additional 1nvestigations way be encceserul in better underatanding the exact charecter of the umasual "dips" ocouring throughout the rate of drying ourves.

Shorwood (26) bae proposed an extensive mathometion Interpretation of the thooretical rate of drying ourre. Ho muthemation discussion 18 offered here for the curves obtalned in this Investigation a the oquations mat necessarily be dorived from the experimentel work. Before obtalning such equations it is necessary to 
deternine exnctis the obaracter of the inte of arying curves, and the faotors upon which the "alpe" are dependent. 
PART IT

VACUUY DRIIEO 
TERORETICAL 
The racum drying of solide constitutes one of those unlt operation of Chemical EnglneerIng about which very little is known. Only a omall mount of work has beon done towards the inveotigation of the theory of reoun drying.

$$
\text { Ridgway (3) conducted atudy of the }
$$
vacurum drying of en inert eolid a11-0-cel. He concluded that the drying tool place in two divislone. The first consiated or a constant rate per1od, during which the 208 in molsture progressed at a constant rate. The second was a ralling rate period, during which the rato of drying was decreased.

Tho conclusions offored by RIdgway (3) aro that during the rirst perlod vater is actually bolling out of the sol1d, and the drying is fairly uniform throughout the cake. A polnt is rosohed at which the surface is falrly dry, after which - vaporation taken place principelly in the inter10r of the cake. This is the falling rate perlod, when diffuation of vapor through the cake is the controlling factor. The essertion that actual bolling occure during the osis stages of vacuun drying is also made by Berthold Blook (27). 
In his experimental work Ridgriag (3) determined the actual temperature withln the cake during the drying. The roeult indiated that the temperature throughout the cake remalned constant unt11 the sol1d was falily $\mathrm{dry}$. The cake temporature then rose raplaly, approeching the actual dryor temperature.

During the conetant temperature period the cake temperature was equal to the temperature at whioh water would boll under the proseure oxistIng within the dryer. Whon the cake became falriy dry throughout the raleing of the cake toupereture unt12 it was equal to the control temperature of the dryor Indicated that the solld hed reached -quilibrium and that the drying had coesed.

In this Investigation 1t wes desired to atudy the mechanism of the drylng of Prassian Blue In vacurum, and to compare the drylng of this commerolal sol1d with the drying of the lnert solld, 11-0-001. It was proposed that the phenomone regarding cake temperatures would be the ame for the two sollds, provided the Prusian Blue cake dried in the oume manner as the inert meterial. 
It wa furthor suggeated that, if the drying ourvo In vacume showed the came "bump" as that found in a1r arying, period when 11ttlo or no drying was taking place, there would appear o correaponding r1so In the cake temperature, tollowed by a decrease whon the evaporation was resumed. 
82

EXPERIMENTAL 


\section{A. APPARATUS and OPERATION}

Tho apparatus used in thls Investigation consisted of a standerd racuum belf dryer, with 1t auxilary oquipment, and other apparatus nocessary in obtaining the experimental data.

The dryer wa a oommerclal four-tray undt construoted by the Buffalo Foundry and Machine Company of Burfa10, How York. F18. 5 10 a photograph of the dryer.

The drying compartment was a singlo heary cast-1ron shell supported on four 10gs. The front of the dryer was a houry door, fastened by screw clampe, and sealed by ganket botwoen it and the dryer sholl. In the door wore two glase port-holes for observing the action within, and a valve for breaking the vacuram.

Material to be driod mas placed on four chalion pand, trenty-four Inchea aquare. Ono of these rested on the bottom of the dryer shell, tho othere on three ahelves. These shelves were ateamjeoketa, connected through manifolds to inlet steam and exhmust 11nes, and were the source of heat for the dryor. 


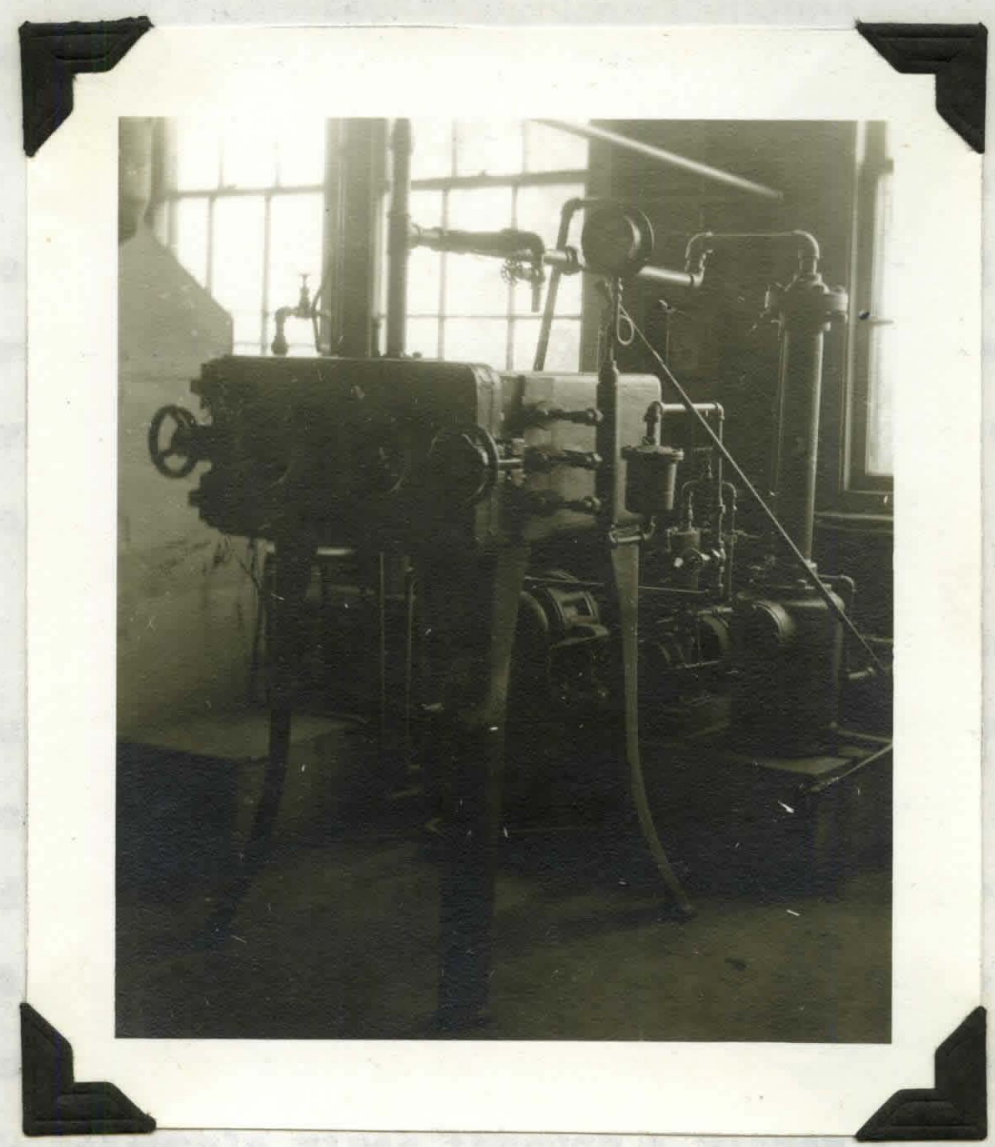

Flgure 5

VACUUM DRYER 
The standard auxillery equipment for the dryer conslatod of water-jecketed surface condenser mounted on a 11quid reoept1ele, and matercooled reaven preip. Tho condenser was located between the aryer and the pump.

A team pressure gauge on the outlet manifold aldod in intaining constant drying temporature. An V-tube manomotor was ut121zed in measuring the racurm. Both steam and recurum wore controlled by means of noedle valves.

In order to obtain data on actual tomperaturea within the dryer ton Copermconstanten thermocouplos were uged. Fig. 6 11Iustrates tho wethod in whioh these couples were brought from the Inside of the drger, through an alr-tight seal, to the Instrument board. This weal was formod passing the thermocouple wirea through a rubber atopper wedged into the branch of the plpe through whioh the recorum was malntaingd.

At the indtrument board the ton Individual Copper-Conatantin cold Junctione were placed In a thermos bottlo in order to mintein them at constant temperature. The oopper leade from the 


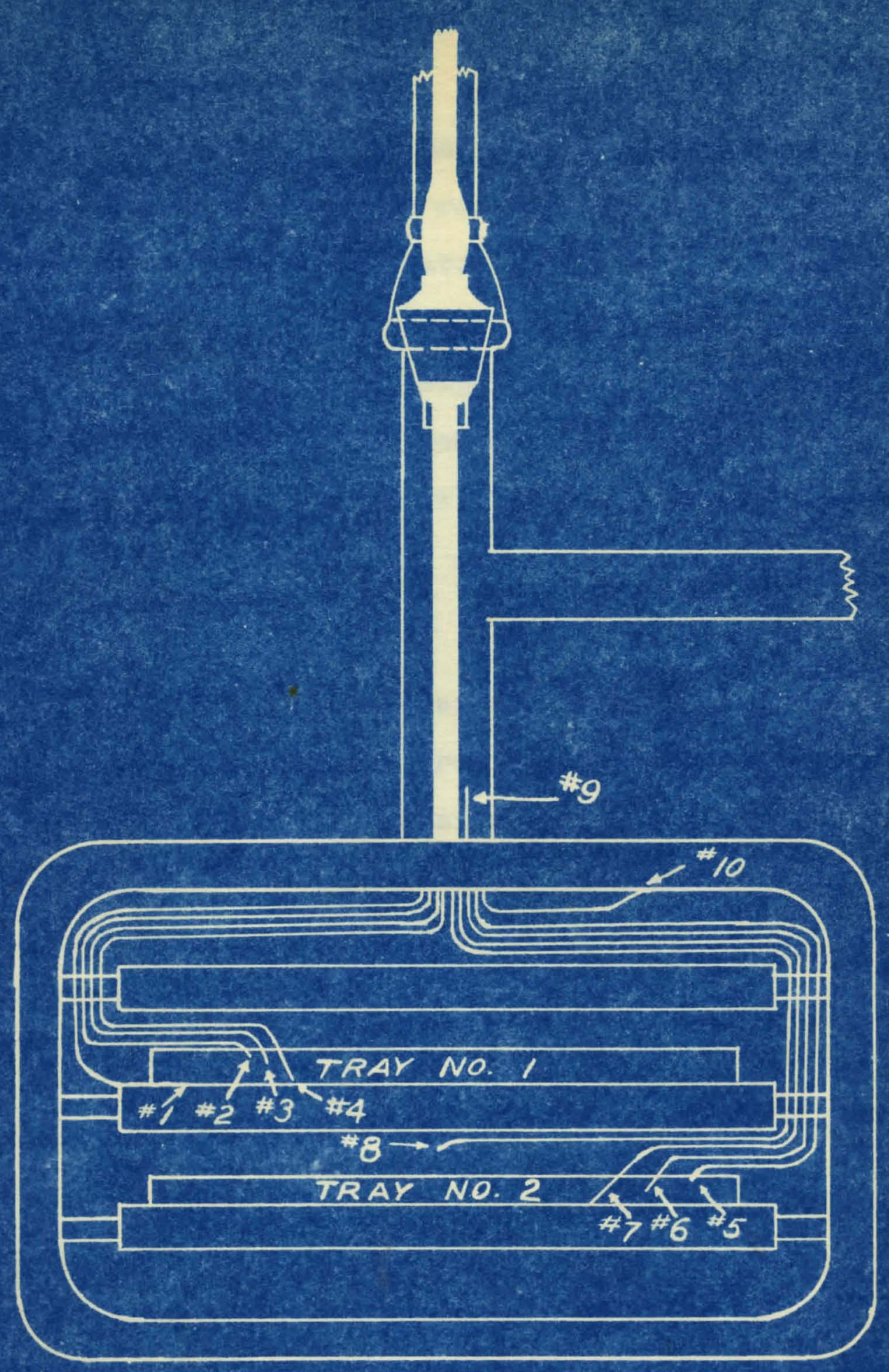

\section{FIGURE 6 \\ SKETCH OF VACUUM DRYER}


cold junctions were connected to two switches, from which copper wires ran to a Leeds and Northrup Potentiometer Indicator. This instrument had a manual cold-end compensatior for correcting the thermocouple cold-end temperature in the thermos bottle to zero degrees Centigrade, whereby it was possible to obtain directly the temperature from the millivolt reading by means of a conversion table (28).

At regular intervals throughout each run samples were taken from the aryer for the purpose of obtaining moisture content data. In the early stages of the runs a cork borer was used in obtalning these samples, unt1l the cake had hardened to the point where a knife was necessary. These samples were placed on watch glasses, weighed and dried in an electric oven before final weighing.

Steam for all of these runs was obtained from a small laboratory boiler. The material used In these tests was a commerclal prussian Blue pigment manufactured by the Kentucky Color and Chemical Company, as described in Part I. 
B. EXPERTMENTAL WORK

A total of twolve experimentul ram vore made at different conditions of tempereture and vacura, as Indieated in Table $X V$. No run woro longer than twelve hours duration, wh1le at those conditione which the material dried in 108 time than that the run was terminated whon the cake temperatures indlceted the material was completely ary.

Two cakes, each equal to one-fourth of a -tandard filter cake were used in ea oh ran. Thene cakes measured eleven inchos square, by about one and ono-fourth inches thick.

Temperatures were obtalned within the aryer by the plaolng of ton thermocouplen as indicated in F18. 6. Ho. 1 wal placed between the top tray and the dryor oholf, and was uad as tho control polnt for mintelning constant temperature. Now, 2,3 and 4 were placed at the top, middle, and bottion reapectively of the top oake, and Nos. 5, 6 and 7 in corresponding positions in the lower cake. No. 8 was placed in the rapor space between the top of the lower cake and the bottom of 


\section{TABLE XV}

\section{TEMPERATURE - VACUUN CONDITIONS OF RUNS}

$\begin{array}{ccc}\begin{array}{c}\text { Run } \\ \text { No. }\end{array} & \begin{array}{c}\text { Tempoum } \\ \text { V. }\end{array} \\ 1 & 100 & 18 \\ 8 & 110 & 18 \\ 3 & 120 & 18 \\ 4 & 130 & 18 \\ 8 & 100 & 28 \\ 6 & 110 & 82 \\ 7 & 180 & 22 \\ 8 & 150 & 22 \\ 9 & 100 & 26 \\ 10 & 110 & 26 \\ 11 & 120 & 86 \\ 12 & 130 & 26\end{array}$


the dryer shelf above. No. 9 wa in the outlet plpe leading to the oondenser and pump, whllo No. 20 gave the temperature of the top of the dryer sholl.

Temperature readings on these thomocouples were taken overy flfteen mimutes throughout each ran. The millivolt roadinga were converted to temperaturea by the converaion teble (28), allowanoes made for the known correction faotors (3), and the correct temperatures in degrees Centigrade recorded directiy in the data book.

Samples for the determination of the mo1sture contents were taken from each trag at half-hour intervals, woighed Imoediately, Aried for forty-e1ght hours at temperature of seventy to elghty degrees Cont1grado, and wolghed again. From the woight of the ary sample and the woight of molsture lost, the grams of moleture per gram of dry solld was coloulated for each samplo.

The fllled trays wore also weighod before and arter each run in order to determine the total molsture lost by the entire cake. 
It was noceseary to break the vacurum in order to obtain the molature content samplos. This was done by opening the valve in the door of the dryer. The total operation of gotting the samplea and returning the dryer to the correct vacuun required fram three to five mimtes for each anpling. 
01

\section{BEsTLrs}


The Information contalned in the Log Sheots was taken directly from the records made of oach run in the laboratory. In particular, the Plant Record Data was the record kept by the Kentuok Color and Chomical Company on the plement used. The IIgure for the Arerage Control Temperature wes an average of the quarter-hour readIngs on the control thermocouple between the top tray and the wols.

Tables of Cake Temperature Data are a rocord of readinge made on thermocouplos in the top, middie and bottom of ench cake. Individual plottings of each thermoconplo (not included) were made, and were the bala for the elimination of a for ralues before onculation of the arorage temperatures for each tray. From these everage temperatures the Cake Temperature Curves were plotted.

Data in the Molsture Content rablos were obtalned from the anall samples taken off oach tray at half-hour intervals. Wolghts of these samples wero expressed as grams molsture per graw dry solld, from which the Dry ing Curves wore plotted. 


\section{TABIS XVI}

\section{IOO SEEES}

Frun 1

Department of Choniond Bnglneering

Speed Sclent1rlo School Univeralty of Loalevil1e

DRIIAO OP PROSSTAY BLUE

\section{Date: $4 / 25 / 34$}

Temperature: $100^{\circ} \mathrm{C}$.

Vecaum: $18^{*} \mathrm{H} 8$.

Plant Reoord Date

Hame: steel Blue

Rx. Wo. 4006

S219 10.1701

Baton No.8

Date struels: $3 / 29 / 34$

Ho. Trays Deled: 1

Date Ins $4 / 25 / 34$
Laboratory Data

Batoh Rod.: $4 / 25 / 34$

Batoh Rtd.: $4 / 27 / 34$

Avs. Control Tomps $90.95^{\circ} \mathrm{C}$.

\section{Tren pate}



$\frac{1}{2}$

5.60
Total $\quad \begin{array}{r}6.00 \\ 1.60\end{array}$

Wt of Cole

After Dry ing

Ibs.

2. 75

8.75

3.60
Wt. Moletare

Lost

Ibe.

8.75

3.28

6.00 
TABLR XVII

CAIE TEMTERATURE DATA

Run 1

TImo

Birs.

0.85

0.80

0.75

1.00

1.25

1.80

1.75

2.00

2.25

2.50

2.75

3.00

3.25

3.50

3.75

4.00

4.25

4.50

4.75

5.00

5.88

5.60

5.75

6.00

6.25

6.50

6.75

7.00

7.25

7.50

7.75

8.00

8.25

8.80

8.75

9.00
Tomperature in ${ }^{\circ} \mathrm{C}$.

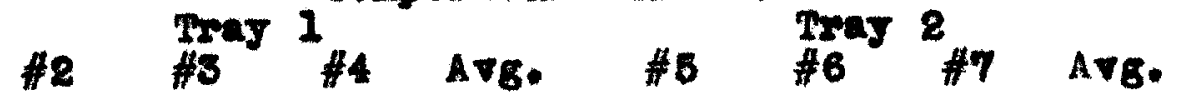
$\begin{array}{llllllll}37.5 & 34.6 & 32.0 & 34.7 & 34.5 & 35.5 & 38.0 & 36.0\end{array}$ $\begin{array}{llllllll}44.5 & 44.0 & 41.5 & 43.5 & 43.0 & 45.5 & 48.0 & 45.5\end{array}$ $86.0 \quad 54.5 \quad 53.0 \quad 54.5$ $63.0 \quad 62.5 \quad 62.0 \quad 68.5$ $67.0 \quad 66.5 \quad 68.0 \quad 67.8$ $69.0 \quad 69.0 \quad 70.0 \quad 69.0$ $70.0 \quad 70.0 \quad 71.0 \quad 70.3$ $71.0 \quad 71.5 \quad 71.6 \quad 71.3$

71.0

71.0

71.0

71.0

71.0

71.0

78.0

7.0

72.0

73.0

72.5

78.0

72.0

73.0

78.5

73.5

73.0

75.075 .0

72.5

72.0

78.0

71.3

71.3

$73.5 \quad 73.0$

$78.0 \quad 78.0$

73.078 .8

73.075 .8

73.073 .0

71.5

71.5

72.0

78.8

$71.5 \quad 71.5$

71.0

71.0

71.0

71.0

69.0

69.0

69.0

69.0

70.0

70.5

70.5

70.3

71.0

71.0

70.5

70.8

70.070 .5

71.5

71.5

71.5

71.5

72.5

71.8

71.5

71.0

71.2

71.571 .8

$71.0 \quad 71.6$

70.5

72.5

69.070 .8

68.0

68.0

71.0

72.5

72.0

72.0

72.8

75.0

71.5

73.0

72.6

70.5

71.0

70.8

72.0
84.0

62.0

60.5

72.0

71.0

71.0

70.5

71.0

71.0

70.0

70.0

69.0

69.5

69.0

68.0

67.5

68.0

68.0

69.0

70.0

68.5

69.0

70.0

70.0

70.0

70.0

71.0

60.0

66.0

69.0

72.0

78.0

72.0

78.0
85.5

61.5

68.0

70.5

70.5

70.5

70.0

70.5

70.0

68.5

68.0

68.5

67.0

67.5

67.0

66.0

66.0

69.0

68.5

69.0

67.8

67.0

68.5

69.5

68.5

68.5

68.5

67.5

65.

67.5

69.5

72.0

69.5

71.0
67.056 .5

63.062 .2

67.568 .0

$71.0 \quad 70.8$

$70.0 \quad 70.5$

70.070 .5

$70.0 \quad 70.2$

70.070 .5

70.570 .5

68.568 .7

67.568 .5

67.568 .3

$67.067,8$

$67.0 \quad 67,8$

67.067 .2

65.566 .3

65.066 .3

68.068 .6

67.568 .3

68.569 .2

67.067 .6

68.068 .0

68.569 .0

68.569 .3

68.569 .0

68.569 .0

68.069 .2

66.067 .5

64.565 .3

66.567 .3

$71.0 \quad 70.8$

$71.0 \quad 71.3$

68.569 .7

71.071 .3 


\section{TABIS XVII (Cont.)}

CATS TEMPERATURE DATA

Run 1

\begin{tabular}{|c|c|c|c|c|c|c|c|c|}
\hline \multirow{2}{*}{ Then } & \multicolumn{8}{|c|}{ Temporatures in ${ }^{\circ} \mathrm{C}$} \\
\hline & $\# 2$ & & $\pi$ & ATE & $\# 8$ & & & AVE. \\
\hline 9.8 & $\begin{array}{l}78.0 \\
72.5 \\
78.0 \\
77.0 \\
78.0 \\
71.0 \\
78.5 \\
78.0 \\
78.0\end{array}$ & $\begin{array}{l}7 \\
77 \\
7 \\
7 \\
7 \\
7 \\
7 \\
7 \\
7 \\
7 \\
6 \\
6\end{array}$ & $\begin{array}{l}7 \\
7 \\
7 \\
7 \\
7 \\
7 \\
7 \\
7 \\
7 \\
7 \\
6 \\
6\end{array}$ & $\begin{array}{l}72.8 \\
71.8 \\
70.8 \\
71.8 \\
71.8 \\
78.8 \\
7.8 \\
72.8 \\
71.8 \\
78.8 \\
66.0 \\
66.2\end{array}$ & & $\begin{array}{l}7 \\
7 \\
7 \\
7 \\
7\end{array}$ & & \\
\hline
\end{tabular}


9

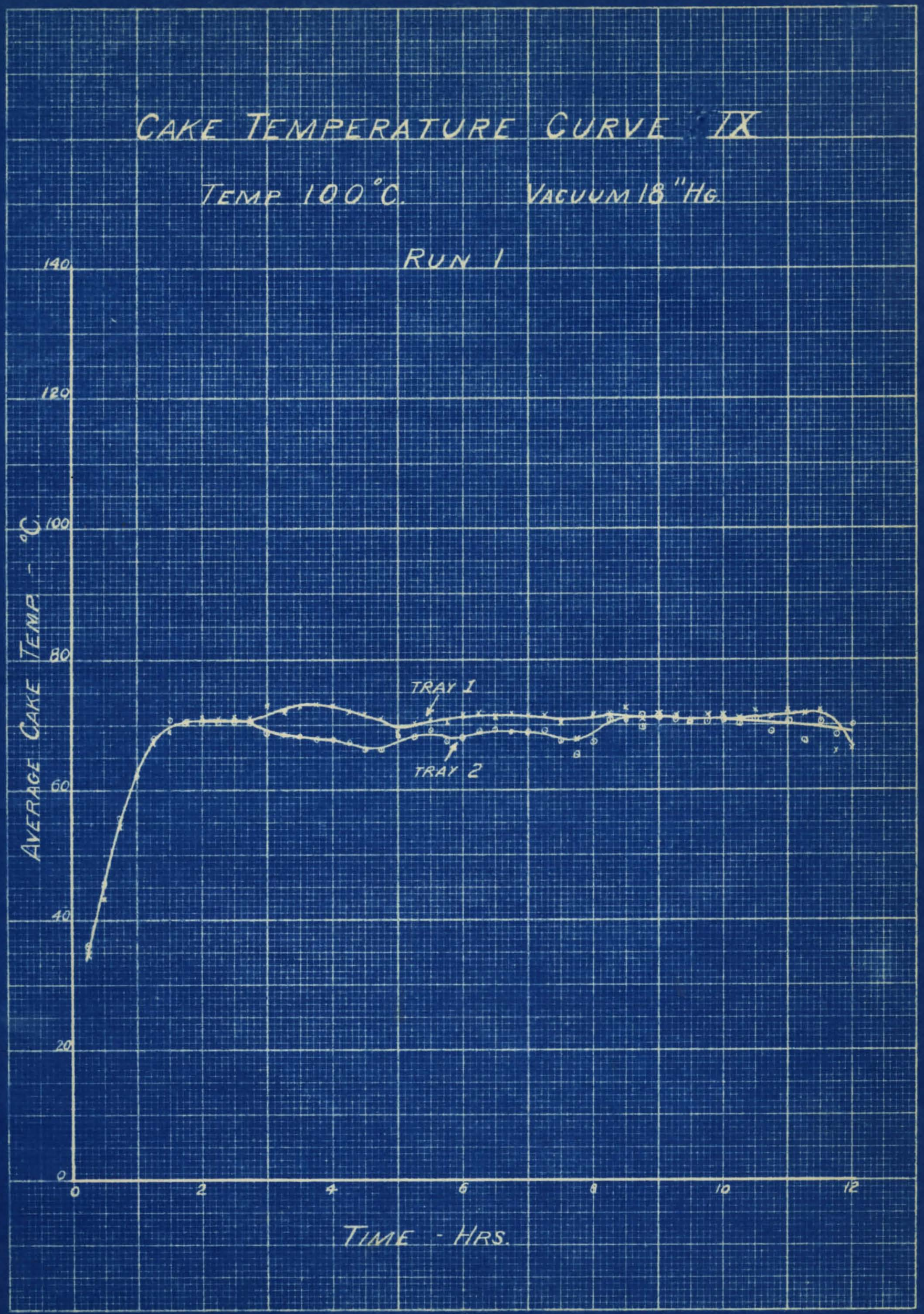


TABIE XVIII

MOISTUKE CONTEYM DATA

nun 1

\begin{tabular}{|c|c|c|c|c|c|}
\hline $\begin{array}{l}\text { PLmo } \\
\text { Hre. }\end{array}$ & $\begin{array}{l}\text { Tyey } \\
\text { No. }\end{array}$ & $\begin{array}{c}\text { Wt. Wet } \\
\operatorname{simp} 2 e \\
\text { Cms. }\end{array}$ & $\begin{array}{l}\text { Wt. Dey } \\
\text { Semple } \\
\text { Cans. }\end{array}$ & $\begin{array}{l}\text { Moloture } \\
\text { Lost } \\
\text { Ann. }\end{array}$ & $\begin{array}{c}\text { Gase Holat } \\
\text { per Om. } \\
\text { Dry solid }\end{array}$ \\
\hline 0 & $\frac{1}{2}$ & $\begin{array}{l}4.56 \\
6.20\end{array}$ & $\begin{array}{l}2.21 \\
2.96\end{array}$ & $\begin{array}{l}2.35 \\
3.24\end{array}$ & $\begin{array}{l}1.065 \\
1.004\end{array}$ \\
\hline 0.5 & $\frac{1}{2}$ & $\begin{array}{l}5.20 \\
4.74\end{array}$ & $\begin{array}{l}2.46 \\
2.37\end{array}$ & $\begin{array}{l}8.74 \\
2.37\end{array}$ & $\frac{1.113}{1.000}$ \\
\hline 2.0 & $\frac{1}{8}$ & $\begin{array}{l}5.10 \\
5.08\end{array}$ & $\begin{array}{l}2.57 \\
2.55\end{array}$ & $\begin{array}{l}2.53 \\
2.53\end{array}$ & $\begin{array}{l}0.985 \\
0.892\end{array}$ \\
\hline 1.5 & $\frac{1}{2}$ & $\begin{array}{l}4.97 \\
5.35\end{array}$ & $\begin{array}{l}2.51 \\
2.68\end{array}$ & $\begin{array}{l}2.46 \\
2.67\end{array}$ & $\begin{array}{l}0.980 \\
0.897\end{array}$ \\
\hline 2.0 & $\frac{1}{2}$ & $\begin{array}{l}4.57 \\
4.50\end{array}$ & $\begin{array}{l}2.42 \\
2.33\end{array}$ & $\begin{array}{l}2.15 \\
2.17\end{array}$ & $\begin{array}{l}0.888 \\
0.938\end{array}$ \\
\hline 2.5 & $\frac{1}{2}$ & $\begin{array}{l}4.51 \\
4.62\end{array}$ & $\begin{array}{l}2.40 \\
2.46\end{array}$ & $\begin{array}{l}2.11 \\
2.16\end{array}$ & $\begin{array}{l}0.879 \\
0.878\end{array}$ \\
\hline 3.0 & $\frac{1}{2}$ & $\begin{array}{l}7.42 \\
5.56\end{array}$ & $\begin{array}{l}4.26 \\
3.16\end{array}$ & $\begin{array}{l}3.16 \\
2.40\end{array}$ & $\begin{array}{l}0.742 \\
0.750\end{array}$ \\
\hline 3.5 & $\frac{1}{2}$ & $\begin{array}{l}5.48 \\
7.42\end{array}$ & $\begin{array}{l}3.17 \\
4.08\end{array}$ & $\begin{array}{l}2.31 \\
3.34\end{array}$ & $\begin{array}{l}0.788 \\
0.818\end{array}$ \\
\hline 4.0 & $\begin{array}{l}1 \\
2\end{array}$ & $\begin{array}{l}5.74 \\
6.67\end{array}$ & $\begin{array}{l}3.49 \\
3.82\end{array}$ & $\begin{array}{l}2.25 \\
2.85\end{array}$ & $\begin{array}{l}0.645 \\
0.746\end{array}$ \\
\hline 4.5 & $\frac{1}{8}$ & $\begin{array}{l}6.18 \\
4.43\end{array}$ & $\begin{array}{l}3.83 \\
2.55\end{array}$ & $\begin{array}{l}2.35 \\
2.88\end{array}$ & $\begin{array}{l}0.614 \\
0.737\end{array}$ \\
\hline 5.0 & $\begin{array}{l}1 \\
2\end{array}$ & $\begin{array}{l}7.98 \\
6.8\end{array}$ & $\begin{array}{l}4.63 \\
3.42\end{array}$ & $\begin{array}{l}3.35 \\
2.76\end{array}$ & $\begin{array}{l}0.784 \\
0.808\end{array}$ \\
\hline 5.5 & $\frac{1}{2}$ & $\begin{array}{l}6.80 \\
4.44\end{array}$ & $\begin{array}{l}3.96 \\
2.49\end{array}$ & $\begin{array}{l}2.85 \\
1.95\end{array}$ & $\begin{array}{l}0.718 \\
0.783\end{array}$ \\
\hline 6.0 & $\frac{1}{2}$ & $\begin{array}{l}6.67 \\
6.56\end{array}$ & $\begin{array}{l}3.92 \\
3.92\end{array}$ & $\begin{array}{l}2.76 \\
2.64\end{array}$ & $\begin{array}{l}0.706 \\
0.673\end{array}$ \\
\hline
\end{tabular}




\section{TABLE XVIII (Cont.)}

MOISTURE CONTENT DATA

Aun 1

\begin{tabular}{|c|c|c|c|c|c|}
\hline $\begin{array}{l}\text { Timo } \\
\text { Bre. }\end{array}$ & $\underset{\mathrm{Ho}}{\mathrm{Tm} \text {. }}$ & $\begin{array}{l}\text { Wt. Wot } \\
\text { Semple } \\
\text { Cans. }\end{array}$ & $\begin{array}{l}\text { Wt. Day } \\
\text { Samplo } \\
\text { Gms. }\end{array}$ & $\begin{array}{c}\text { Molature } \\
\text { Lost } \\
\text { Cma }\end{array}$ & $\begin{array}{c}\text { Gane, Noidet. } \\
\text { per Cas, } \\
\text { Dan solid }\end{array}$ \\
\hline 6.5 & $\frac{1}{2}$ & $\begin{array}{l}6.25 \\
7.32\end{array}$ & $\begin{array}{l}3.78 \\
4.84\end{array}$ & $\begin{array}{l}8.47 \\
8.78\end{array}$ & $\begin{array}{l}0.653 \\
0.612\end{array}$ \\
\hline 7.0 & $\frac{1}{2}$ & $\begin{array}{l}6.45 \\
7.78\end{array}$ & $\begin{array}{l}3.88 \\
4,31\end{array}$ & $\begin{array}{l}2.68 \\
5,47\end{array}$ & $\begin{array}{l}0.683 \\
0.605\end{array}$ \\
\hline 7.6 & $\begin{array}{l}1 \\
8\end{array}$ & $\begin{array}{l}6.70 \\
5.87\end{array}$ & $\begin{array}{l}4.53 \\
3.39\end{array}$ & $\begin{array}{l}2.17 \\
2.48\end{array}$ & $\begin{array}{l}0.478 \\
0.751\end{array}$ \\
\hline 8.0 & $\frac{1}{2}$ & $\begin{array}{l}5.10 \\
4.86\end{array}$ & $\begin{array}{l}3.95 \\
3.17\end{array}$ & $\begin{array}{l}2.27 \\
1.69\end{array}$ & $\begin{array}{l}0,399 \\
0,583\end{array}$ \\
\hline 8.5 & $\begin{array}{l}1 \\
2\end{array}$ & $\begin{array}{l}4.54 \\
5.51\end{array}$ & $\begin{array}{l}2.95 \\
3.39\end{array}$ & $\begin{array}{l}1.59 \\
2.12\end{array}$ & $\begin{array}{l}0.630 \\
0.625\end{array}$ \\
\hline 8.0 & $\frac{1}{8}$ & $\begin{array}{l}3.27 \\
5.82\end{array}$ & $\begin{array}{l}2.25 \\
3.88\end{array}$ & $\begin{array}{l}1.08 \\
1.04\end{array}$ & $\begin{array}{l}0.468 \\
0.500\end{array}$ \\
\hline 0.5 & $\frac{2}{8}$ & $\begin{array}{l}2.35 \\
6.87\end{array}$ & $\begin{array}{r}1.89 \\
4.31\end{array}$ & $\begin{array}{l}0.46 \\
1.86\end{array}$ & $\begin{array}{l}0,844 \\
0,465\end{array}$ \\
\hline 10.0 & $\frac{1}{2}$ & $\begin{array}{l}5.11 \\
3.51\end{array}$ & $\begin{array}{l}4.25 \\
2.37\end{array}$ & $\begin{array}{l}0.86 \\
1.14\end{array}$ & $\begin{array}{l}0.202 \\
0.488\end{array}$ \\
\hline 10.5 & $\frac{1}{2}$ & $\begin{array}{l}2.99 \\
4.84\end{array}$ & $\begin{array}{l}2.08 \\
3.27\end{array}$ & $\begin{array}{l}0.84 \\
1.87\end{array}$ & $\begin{array}{l}0.458 \\
0.388\end{array}$ \\
\hline 21.0 & $\frac{2}{2}$ & $\begin{array}{l}6.41 \\
5.75\end{array}$ & $\begin{array}{l}4.99 \\
4.20\end{array}$ & $\begin{array}{l}1.42 \\
1.85\end{array}$ & $\begin{array}{l}0.284 \\
0.360\end{array}$ \\
\hline 11.6 & $\begin{array}{l}2 \\
8\end{array}$ & $\begin{array}{l}7.00 \\
7.10\end{array}$ & $\begin{array}{l}5.50 \\
5.26\end{array}$ & $\begin{array}{l}1.85 \\
1.84\end{array}$ & $\begin{array}{l}0.386 \\
0.350\end{array}$ \\
\hline 12.0 & $\frac{1}{8}$ & $\begin{array}{l}3.41 \\
6.18\end{array}$ & $\begin{array}{l}2.55 \\
5.48\end{array}$ & $\begin{array}{l}0.86 \\
0.70\end{array}$ & $\begin{array}{l}0.898 \\
0.188\end{array}$ \\
\hline
\end{tabular}




\section{TABLI XIX}

IoO SHBis

Run 8

Dopartinont of Chomionl Bnglnoering

spoed solontiflo sohool UnIvereity of Loulevilie

DFIIUC OF PROSSIAX BLUE

Date: $4 / 28 / 34$

Temperature, $110^{\circ} \mathrm{C}$. Vecuin: $18^{7}$ H8.

PInnt Record Date

Namo: Steol BIne

Rx. No.: 4006

SIIp N0.2 731

Batoh Ho.: 4

Date struok: 3/29/34

Ho. Tray Deled: 1

De to Ini $4 / 27 / 54$
Iaboratory Data

Batch Rod.: $4 / 27 / 34$

Butch Rtd.: $4 / 30 / 54$

Avg. Control Temp. $109.82^{\circ} \mathrm{C}$.

Tray Data

\begin{tabular}{|c|c|}
\hline $\begin{array}{l}\text { Tras } \\
\text { Xo. }\end{array}$ & $\begin{array}{l}\text { Wt. of cake } \\
\text { Before pery Ins } \\
\text { Ibs. }\end{array}$ \\
\hline & $\begin{array}{r}7.80 \\
7.25 \\
17.16\end{array}$ \\
\hline
\end{tabular}

wt. of Cake
After Drying
2bs.

4.00

$\frac{4.00}{8.00}$
Wt. Mo1sture Lont 2bs.

3.50

3.25 
TABIX $\boldsymbol{x}$

CAXR TEMPERA TURE DATA

Run 2






\section{TABLE XX (Cont.) \\ CATE THUPERATURE DATA}

Run 2

Timo

. Brre.

Tomperaturea in ${ }^{\circ} \mathrm{C}$.

42 Tray $^{2} 4$ ATE. \#5 $\$ 6$

8

$9.86 \quad 73.8 \quad 78.5 \quad 73.0 \quad 73.0$

9.50

78.0

74.0

74.0

74.3

78.8

9.7573 .0

10.00

74.0

7.5

72,5

72.0

73.573 .3

71.571 .8

20.50

72,0

74.0

70.5

73.5

73.073 .5

72.872 .8

$20.75 \quad 72.5$

21.00

74.0

72.5

75.5

21.25

78.5

73.0

73.0

11. 50

78.5

21.76

74.0

7.5

73.0

73.5

73.8

73.2

73.2

73.5

72.0

76.0

$73.7 \quad 73.7$

$77.0 \quad 75.0 \quad 75.8 \quad 75.8$

$76.5 \quad 75.0 \quad 74.5 \quad 74.7$

$76.074 .6 \quad 74.0 \quad 74.0$

$75.8 \quad 74.0 \quad 73.5 \quad 74.8$

$7020 \quad 75.5 \quad 78.5 \quad 75.0$

$74.0 \quad 74.0 \quad 75.0 \quad 73.7$

$78.0 \quad 72.5 \quad 73.0 \quad 72.8$

$74.0 \quad 7.0 \quad 7.5 \quad 7.5$

$74.5 \quad 72.5 \quad 72.5 \quad 70.2$

$74.0 \quad 75.5 \quad 73.5 \quad 75.7$

12,00

76.0

$74.0 \quad 73.5 \quad 7.5 \%$

$75.5 \quad 75.5 \quad 75,0 \quad 74,0$ 
CAKE TEMPERATURE CURVE II Temp $110^{\circ} \mathrm{C}$. Vacuum $18^{\prime \prime} \mathrm{HG}$.

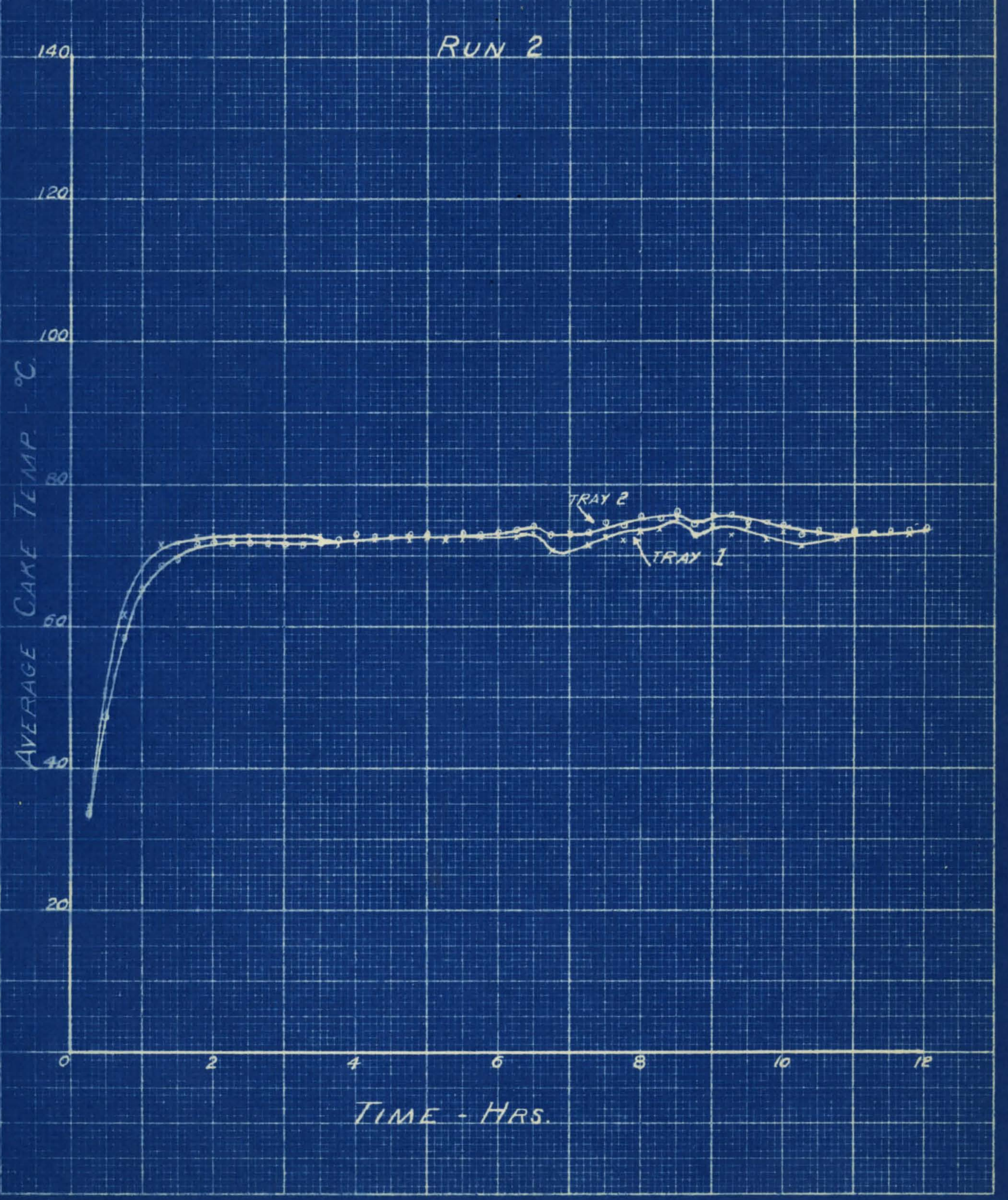


TABLE XXI

MOTSTURE CONTENT DATA

Pan 2

Tim Tres Nt. Wet Wt. Des Molsture Cus. Molet.

Hra. Ho. Sample Sample Lout

Cms. Che. Cms.

per bin.

Dry solid

\begin{tabular}{|c|c|c|c|c|}
\hline 0 & $\frac{1}{2}$ & $\begin{array}{l}5.386 \\
5.788\end{array}$ & $\begin{array}{l}8.770 \\
2.808\end{array}$ & $\begin{array}{l}2.618 \\
8.930\end{array}$ \\
\hline 0.5 & $\frac{1}{2}$ & $\begin{array}{l}4.870 \\
5.948\end{array}$ & $\begin{array}{l}8.658 \\
3.207\end{array}$ & $\begin{array}{l}8.217 \\
8.751\end{array}$ \\
\hline 1.0 & $\frac{1}{8}$ & $\begin{array}{l}6.091 \\
5.031\end{array}$ & $\begin{array}{l}3.798 \\
8.752\end{array}$ & $\begin{array}{l}9.898 \\
8.280\end{array}$ \\
\hline 1.5 & $\frac{1}{2}$ & $\begin{array}{l}6.780 \\
5.680\end{array}$ & $\begin{array}{l}3.791 \\
2.899\end{array}$ & $\begin{array}{l}8.997 \\
8.721\end{array}$ \\
\hline 2.0 & $\begin{array}{l}1 \\
2\end{array}$ & $\begin{array}{l}7.281 \\
7.325\end{array}$ & $\begin{array}{c}4.171 \\
5.986\end{array}$ & $\begin{array}{l}3.080 \\
0.037\end{array}$ \\
\hline 2.5 & $\frac{1}{2}$ & $\begin{array}{l}6.484 \\
5.965\end{array}$ & $\begin{array}{l}3.613 \\
5.377\end{array}$ & $\begin{array}{l}2.811 \\
2.586\end{array}$ \\
\hline 3.0 & $\frac{1}{2}$ & $\begin{array}{l}6.614 \\
5.006\end{array}$ & $\begin{array}{l}3.980 \\
2.021\end{array}$ & $\begin{array}{l}8.698 \\
8.085\end{array}$ \\
\hline 3.5 & $\frac{1}{8}$ & $\begin{array}{l}6.825 \\
8.754\end{array}$ & $\begin{array}{l}4.077 \\
5.055\end{array}$ & $\begin{array}{l}2.736 \\
5.699\end{array}$ \\
\hline 4.0 & $\frac{1}{2}$ & $\begin{array}{l}7.697 \\
7.659\end{array}$ & $\begin{array}{r}4.008 \\
4.706\end{array}$ & $\begin{array}{l}2.894 \\
2.965\end{array}$ \\
\hline 4.5 & $\frac{1}{8}$ & $\begin{array}{c}9.100 \\
6.186\end{array}$ & $\begin{array}{l}5.755 \\
3.909\end{array}$ & $\begin{array}{l}3.347 \\
2.877\end{array}$ \\
\hline 5.0 & $\frac{1}{2}$ & $\begin{array}{l}6.695 \\
6.210\end{array}$ & $\begin{array}{r}4.964 \\
5.961\end{array}$ & $\begin{array}{l}8.431 \\
2.049\end{array}$ \\
\hline 5.5 & $\frac{2}{2}$ & $\begin{array}{r}7.710 \\
10.048\end{array}$ & $\begin{array}{l}4.896 \\
6.534\end{array}$ & $\begin{array}{l}2.814 \\
3.514\end{array}$ \\
\hline 6.0 & $\frac{1}{2}$ & $\begin{array}{l}8.457 \\
8.827\end{array}$ & $\begin{array}{l}5.788 \\
6.044\end{array}$ & $\begin{array}{l}8.689 \\
2.883\end{array}$ \\
\hline
\end{tabular}


TABLE XXI (Cont.)

MOISTURE CONTEMP DA

Run 2

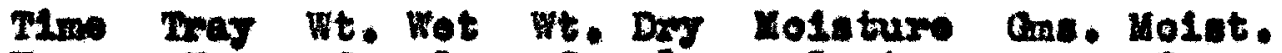

Hre. Ho. Sample Samplo Loat Oma. Oma. ans. per Cn. Dary Solld

\begin{tabular}{|c|c|c|c|c|c|}
\hline 6.8 & $\begin{array}{l}2 \\
2\end{array}$ & $\begin{array}{l}6.671 \\
6.024\end{array}$ & $\begin{array}{l}4.608 \\
4.081\end{array}$ & $\begin{array}{l}2.063 \\
1.970\end{array}$ & $\begin{array}{l}0.448 \\
0.487\end{array}$ \\
\hline 7.0 & $\begin{array}{l}1 \\
2\end{array}$ & $\begin{array}{l}6.701 \\
5.341\end{array}$ & $\begin{array}{l}5.101 \\
3.905\end{array}$ & $\begin{array}{l}1.300 \\
1.456\end{array}$ & $\begin{array}{l}0.241 \\
0.367\end{array}$ \\
\hline 7.5 & $\frac{1}{2}$ & $\begin{array}{l}6.694 \\
7.717\end{array}$ & $\begin{array}{l}6.318 \\
5.683\end{array}$ & $\begin{array}{l}1.576 \\
8.034\end{array}$ & $\begin{array}{l}0.259 \\
0.358\end{array}$ \\
\hline 8.0 & $\begin{array}{l}1 \\
8\end{array}$ & $\begin{array}{l}3.795 \\
6.874\end{array}$ & $\begin{array}{l}\$ .131 \\
5.589\end{array}$ & $\begin{array}{l}0,684 \\
1,845\end{array}$ & $\begin{array}{l}0.313 \\
0.234\end{array}$ \\
\hline 8.5 & $\frac{1}{2}$ & $\begin{array}{l}6.088 \\
4.608\end{array}$ & $\begin{array}{l}5.951 \\
3.067\end{array}$ & $\begin{array}{l}0.971 \\
0.636\end{array}$ & $\begin{array}{l}0.265 \\
0.160\end{array}$ \\
\hline 9.0 & $\frac{1}{2}$ & $\begin{array}{l}7.480 \\
6.485\end{array}$ & $\begin{array}{l}5.904 \\
8.871\end{array}$ & $\begin{array}{l}2.486 \\
2.164\end{array}$ & $\begin{array}{l}0.249 \\
0.281\end{array}$ \\
\hline 0.5 & $\frac{1}{8}$ & $\begin{array}{l}6.792 \\
5.078\end{array}$ & $\begin{array}{l}5.827 \\
4.245\end{array}$ & $\begin{array}{l}0.875 \\
0.835\end{array}$ & $\begin{array}{l}0.168 \\
0.197\end{array}$ \\
\hline 10.0 & $\frac{1}{2}$ & $\begin{array}{l}4.901 \\
6.206\end{array}$ & $\begin{array}{l}4.800 \\
4.856\end{array}$ & $\begin{array}{l}0.701 \\
1.270\end{array}$ & $\begin{array}{l}0.167 \\
0.257\end{array}$ \\
\hline 10.5 & $\begin{array}{l}1 \\
2\end{array}$ & $\begin{array}{l}5.722 \\
8.519\end{array}$ & $\begin{array}{l}6.025 \\
6.958\end{array}$ & $\begin{array}{l}0.697 \\
1.867\end{array}$ & $\begin{array}{l}0.139 \\
0.228\end{array}$ \\
\hline 11.0 & $\begin{array}{l}1 \\
2\end{array}$ & $\begin{array}{l}6.212 \\
6.800\end{array}$ & $\begin{array}{l}5.477 \\
5.400\end{array}$ & $\begin{array}{l}0.711 \\
1.080\end{array}$ & $\begin{array}{l}0.135 \\
0.2008\end{array}$ \\
\hline 21.5 & $\begin{array}{l}2 \\
2\end{array}$ & $\begin{array}{l}6.383 \\
5.374\end{array}$ & $\begin{array}{l}5.889 \\
4.790\end{array}$ & $\begin{array}{l}0.494 \\
0.584\end{array}$ & $\begin{array}{l}0.084 \\
0.188\end{array}$ \\
\hline 12.0 & $\begin{array}{l}1 \\
2\end{array}$ & $\begin{array}{l}6.988 \\
5.286\end{array}$ & $\begin{array}{l}6.380 \\
4.749\end{array}$ & $\begin{array}{l}0.558 \\
0.557\end{array}$ & $\begin{array}{l}0.087 \\
0.113\end{array}$ \\
\hline
\end{tabular}




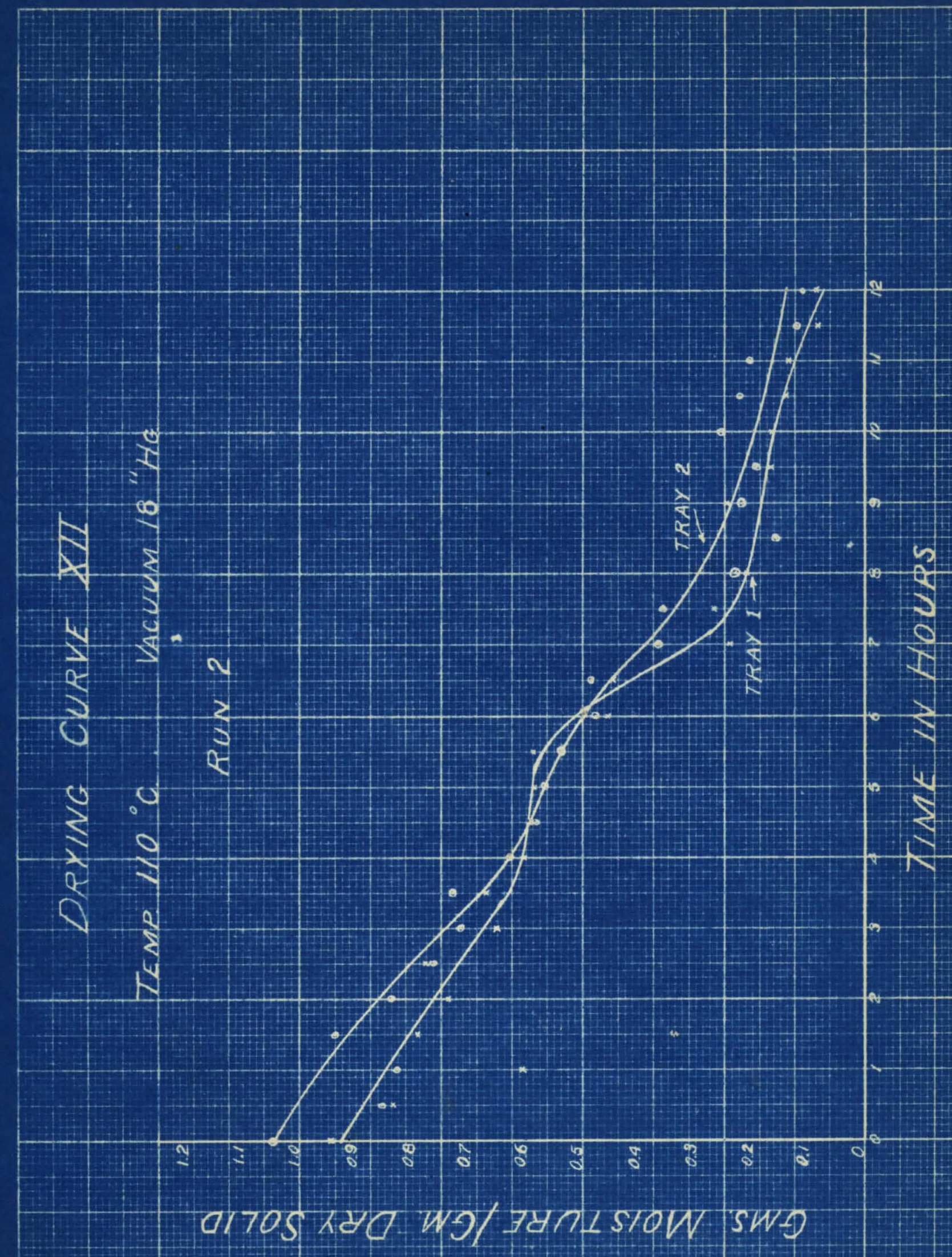




\section{TABLS XXII}

LOQ SHEET

Ran 3

Departmont of Chimical Bnglnooring

Speed Selontiflo Sohool Untroralty of Loulev1110

$$
\text { DRYIHO OF PRUSSIA BLOB }
$$

Date: $5 / 3 / 34$

Temperature: $180^{\circ} \mathrm{C}$.

Plant Rocord Deta

Name: Stoel Blue

Rx. No.8 4006

S11p No.: 731

Beton No.: 4

Date struck: $3 / 29 / 34$

INo. Traj: Dried: 5

Deto In: $4 / 30 / 34$
Vacuin: 18" $\mathrm{HB}$.

Tray Data

\begin{tabular}{|c|c|c|c|}
\hline $\begin{array}{c}\text { Tras } \\
\text { Mo. }\end{array}$ & $\begin{array}{l}\text { Wt. of Cake } \\
\text { Before Deyling } \\
\text { lbu. }\end{array}$ & $\begin{array}{l}\text { Wt. of Cake } \\
\text { After Deying } \\
\text { Ibs. }\end{array}$ & $\begin{array}{l}\text { Wt. Holsture } \\
\text { Iost } \\
\text { Ibes. }\end{array}$ \\
\hline 3 & $\begin{array}{r}6.86 \\
6.75 \\
15.00\end{array}$ & $\begin{array}{l}3.25 \\
3.25 \\
6.50\end{array}$ & $\begin{array}{l}3.00 \\
3.50 \\
6.50\end{array}$ \\
\hline
\end{tabular}


MABIE XXIII

CAKS TBMPERATURE DATA

Prun 3

TIm

Hire.

Tras 1

Ramperetures in ${ }^{\circ} \mathrm{C}$. \#2 \#8 \#4 Avg. \#5 \#6 \#7 AVg.

0.85

35.0

33.5

$36.5 \quad 35.0$

36.0

37.5

37.5

37.0

0.50

52.5

58.0

59.5

54.7

50.0

50.0

58.0

50.8

0.75

71.0

71.0

$71.0 \quad 72.0$

66.0

66.5

68.0

72.5

$74.0 \quad 72.8$

70.5

73.5

1.85

73.0

74.0

74.075 .3

72.0

75.5

74.0

66.8

1.50

74.0

74.0

$74.0 \quad 74.0$

72.5

74.0

74.0

78.7

1.75

74.0

74.0

$74.0 \quad 74.0$

75.0

78.5

73.5

75.0

2.00

74.5

74.5

$74.0 \quad 74.3$

72.5

74.0

74.0

73.3

2.25

74.0

74.5

$74.0 \quad 74.3$

72.5

75.5

73.5

73.5

2.50

74.0

75.0

$74.0 \quad 74.0$

72.5

74.0

73.5

73.3

2.76

74.0

74.5

$74.0 \quad 74.2$

78.0

74.0

73.5

73.2

3.00

74.0

74.0

$74.0 \quad 74.0$

73.0

74.0

73.5

73.3

3.25

74.0

74.5

74.0

74.2

73.0

74.0

73.5

73.5

$\$ .76$

74.0

74.0

$74.0 \quad 74.0$

13.0

74.0

73.5

73.5

4.00

74.0

7.0

74.0

74.0

73.0

73.5

73.5

73.5

4.86

73.5

73.5

75.5

75.6

73.5

73.0

70.5

13.5

4.75

74.0

74.0

74.0

74.0

74.0

74.5

73.5

75.3

5.00

14.0

74.0

74.0

$7 \times 0$

74.0

74.5

78.0

75.3

5.85

74.0

74.0

74.0

74.0

74.0

74.5

73.0

7.2

5.80

78.0

70.5

74.0

74.2

74.0

74.5

73.5

74.0

8.75

74.0

74.5

$74.0 \quad 74.5$

74.0

75.5

73.0

74.0

6.00

75.5

74.0

74.8

74.0

75.5

73.5

73.8

75.0

74.6

74.0

74.5

74.0

76.5

74.5

74.0

6.80

75.0

74.5

74.074 .5

74.5

74.5

73.5

74.7

6.76

76.0

76.0

75.5

75.8

76.0

75.5

76.5

75.0

7.00

77.0

77.5

77.5

77.3

76.5

75.5

74.5

74.0

7.85

76.5

76.0

77.0

76.5

74.0

77.5

70.5

74.7

7.80

78.0

78.0

74.0

74.5

74.0

75.5

77.0

77.0

8.00

75.0

75.0

74.0

74.3

74.0

74.0

75.6

77.0

8.26

75.0

74.6

74.0

74.3

74.0

74.0

74.5

74.3

8.50

74.0

74.0

74.5

74.0

74.0

75.5

74.2

8.75

75.0

7.0

73.0

73.3

78.0

73.5

75.5

74.5

9.00

77.0

74,0

74.0

74.0

72.0

70.5

75.5

74.5

74.3

$78.0 \quad 76.3$

78.0

72.5

74.0

78.8

74.072 .5

708071.5 
TABLE XXIII (cont.)

CAKE TEMPERATURE DATA

Ran 8

\begin{tabular}{|c|c|c|c|c|c|c|c|c|}
\hline & & & & $a_{t}$ & in & & & \\
\hline & $\frac{4}{42}$ & 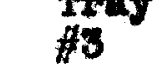 & 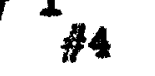 & $A v 8$. & $\# 5$ & & $\# 7$ & AVE. \\
\hline $\begin{array}{l}9.28 \\
9.50 \\
9.75 \\
10.00 \\
10.25 \\
10.50 \\
10.75 \\
11.00 \\
1.28\end{array}$ & $\begin{array}{r}93.0 \\
85.0 \\
88.0 \\
95.0 \\
105.0 \\
112.5 \\
11.48 \\
128.0 \\
120.0 \\
121.0\end{array}$ & $\begin{array}{r}77.5 \\
85.5 \\
91.5 \\
99.5 \\
106.5 \\
114.0 \\
113.5 \\
116.5 \\
180.0 \\
120.5\end{array}$ & $\begin{array}{r}89.0 \\
97.0 \\
101.0 \\
107.0 \\
110.5 \\
117.0 \\
113.0 \\
117.0 \\
118.5\end{array}$ & $\begin{array}{r}83.8 \\
89.8 \\
95.5 \\
100.5 \\
107.5 \\
104.5 \\
128.8 \\
117.8 \\
119.5 \\
120.7\end{array}$ & $\begin{array}{r}77.0 \\
81.0 \\
83.0 \\
90.0 \\
98.5 \\
105.0 \\
107.0 \\
113.0 \\
116.0 \\
119.0\end{array}$ & $\begin{array}{r}76.0 \\
81.5 \\
88.5 \\
93.5 \\
100.5 \\
106.5 \\
107.5 \\
113.0 \\
118.5 \\
118.5\end{array}$ & $\begin{array}{r}78.5 \\
87.0 \\
91.0 \\
98.0 \\
108.5 \\
107.0 \\
108.5 \\
113.5 \\
115\end{array}$ & $\begin{array}{r}77.2 \\
83.2 \\
86.5 \\
93.8 \\
100.5 \\
106.2 \\
107.7 \\
113.8 \\
115.5\end{array}$ \\
\hline
\end{tabular}


CAKE TEMPERATURE CURVE XIII TEMP $120^{\circ} \mathrm{C}$ VACUUM $18^{\circ} \mathrm{HG}$

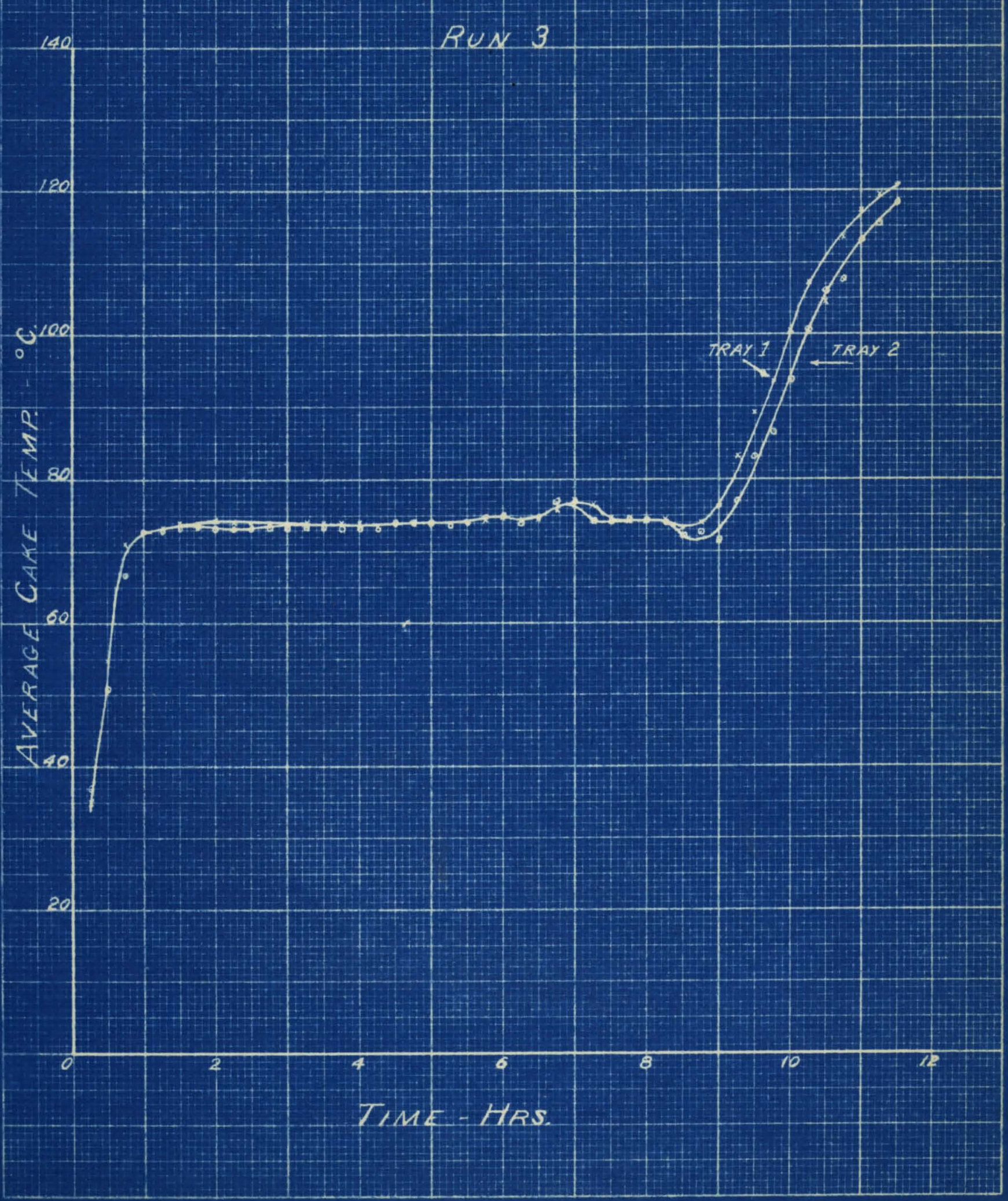


TBLE XXIV

HOISTUPE COMTENT DATA

than 5

\begin{tabular}{|c|c|c|c|c|c|}
\hline $\begin{array}{l}\text { Time } \\
\text { Hre. }\end{array}$ & Tray & $\begin{array}{c}\text { wt. Wot } \\
\text { Sample } \\
\text { Comp. }\end{array}$ & $\begin{array}{c}\text { wt. Dry } \\
\text { simplo } \\
\text { Cone. }\end{array}$ & $\begin{array}{l}\text { Molotrure } \\
\text { Lost } \\
\text { Onas. }\end{array}$ & $\begin{array}{l}\text { Cone. Molit } \\
\text { per Om. } \\
\text { Deng solid }\end{array}$ \\
\hline 0 & $\frac{1}{2}$ & $\begin{array}{l}5.800 \\
4.766\end{array}$ & $\begin{array}{l}2.738 \\
2.375\end{array}$ & $\begin{array}{l}2.777 \\
2.381\end{array}$ & $\begin{array}{l}1.018 \\
1.008\end{array}$ \\
\hline 0.8 & $\frac{1}{2}$ & $\begin{array}{l}3.601 \\
3.826\end{array}$ & $\begin{array}{l}1.920 \\
1.084\end{array}$ & $\begin{array}{l}1.781 \\
1.878\end{array}$ & $\begin{array}{l}0.858 \\
0.888\end{array}$ \\
\hline 1.0 & $\frac{1}{2}$ & $\begin{array}{l}5.688 \\
3.884\end{array}$ & $\begin{array}{l}3.046 \\
8.093\end{array}$ & $\begin{array}{l}2.506 \\
1.751\end{array}$ & $\begin{array}{l}0.860 \\
0.888\end{array}$ \\
\hline 1.5 & $\frac{1}{8}$ & $\begin{array}{l}5.847 \\
7.816\end{array}$ & $\begin{array}{l}3.267 \\
5.850\end{array}$ & $\begin{array}{r}2.580 \\
3.387\end{array}$ & $\begin{array}{l}0.799 \\
0.869\end{array}$ \\
\hline 2.0 & $\frac{1}{8}$ & $\begin{array}{l}4.334 \\
5.496\end{array}$ & $\begin{array}{l}2.650 \\
3.185\end{array}$ & $\begin{array}{l}1.684 \\
2.341\end{array}$ & $\begin{array}{l}0.655 \\
0.748\end{array}$ \\
\hline 2.5 & $\frac{1}{8}$ & $\begin{array}{l}6.754 \\
6.466\end{array}$ & $\begin{array}{l}3.954 \\
3.579\end{array}$ & $\begin{array}{l}8.800 \\
2.887\end{array}$ & $\begin{array}{l}0.608 \\
0.807\end{array}$ \\
\hline 3.0 & $\frac{1}{2}$ & $\begin{array}{l}5.678 \\
5.275\end{array}$ & $\begin{array}{l}5.472 \\
3.212\end{array}$ & $\begin{array}{l}2.800 \\
2.065\end{array}$ & $\begin{array}{l}0.634 \\
0.643\end{array}$ \\
\hline 3.5 & $\begin{array}{l}1 \\
2\end{array}$ & $\begin{array}{l}7.787 \\
6.046\end{array}$ & $\begin{array}{l}5.047 \\
3.668\end{array}$ & $\begin{array}{l}2.680 \\
2.384\end{array}$ & $\begin{array}{l}0.551 \\
0.658\end{array}$ \\
\hline 4.0 & $\begin{array}{l}1 \\
8\end{array}$ & $\begin{array}{l}5.327 \\
5.416\end{array}$ & $\begin{array}{l}3.656 \\
3.426\end{array}$ & $\begin{array}{l}1.671 \\
1.990\end{array}$ & $\begin{array}{l}0.457 \\
0.580\end{array}$ \\
\hline 4.8 & $\begin{array}{l}1 \\
2\end{array}$ & $\begin{array}{l}6.384 \\
6.721\end{array}$ & $\begin{array}{l}4.458 \\
4.505\end{array}$ & $\begin{array}{l}1.866 \\
1.816\end{array}$ & $\begin{array}{l}0.428 \\
0.370\end{array}$ \\
\hline 5.0 & $\begin{array}{l}1 \\
2\end{array}$ & $\begin{array}{l}6.038 \\
7.844\end{array}$ & $\begin{array}{l}4.584 \\
5.293\end{array}$ & $\begin{array}{l}1.485 \\
2.551\end{array}$ & $\begin{array}{l}0.318 \\
0.488\end{array}$ \\
\hline 5.5 & $\frac{1}{2}$ & $\begin{array}{l}5.059 \\
6.351\end{array}$ & $\begin{array}{l}3.574 \\
4.409\end{array}$ & $\begin{array}{l}1.485 \\
2.042\end{array}$ & $\begin{array}{l}0.416 \\
0.441\end{array}$ \\
\hline 6.0 & $\frac{1}{2}$ & $\begin{array}{l}4.736 \\
3.186\end{array}$ & $\begin{array}{l}4.022 \\
3.733\end{array}$ & $\begin{array}{l}0.714 \\
1.458\end{array}$ & $\begin{array}{l}0.178 \\
0.380\end{array}$ \\
\hline
\end{tabular}


mabes XXN (Cont.)

MOTSTURE CONTEST DATA

Pan 3

\begin{tabular}{|c|c|c|c|c|c|}
\hline $\begin{array}{l}\text { Timo } \\
\text { Hra. }\end{array}$ & To. & $\begin{array}{c}\text { Wt. Wot } \\
\text { Simple } \\
\text { Ome. }\end{array}$ & $\begin{array}{c}\text { Wt. Dery } \\
\text { simplo } \\
\text { Oane. }\end{array}$ & $\begin{array}{l}\text { Molature } \\
\text { Loet } \\
\text { Cont. }\end{array}$ & $\begin{array}{l}\text { Con Molet. } \\
\text { per Omb } \\
\text { Dey solid }\end{array}$ \\
\hline 6.8 & $\frac{1}{8}$ & $\begin{array}{l}4.001 \\
4.934\end{array}$ & $\begin{array}{l}3.676 \\
3.661\end{array}$ & $\begin{array}{l}0.328 \\
1.875\end{array}$ & $\begin{array}{l}0.088 \\
0.0348\end{array}$ \\
\hline 7.0 & $\frac{1}{2}$ & $\begin{array}{l}5.198 \\
5.845\end{array}$ & $\begin{array}{l}4.658 \\
4.856\end{array}$ & $\begin{array}{l}0.558 \\
1.287\end{array}$ & $\begin{array}{l}0.220 \\
0.282\end{array}$ \\
\hline 7.5 & $\begin{array}{l}1 \\
8\end{array}$ & $\begin{array}{l}6.289 \\
4.467\end{array}$ & $\begin{array}{l}4.824 \\
3.634\end{array}$ & $\begin{array}{l}1.405 \\
0.858\end{array}$ & $\begin{array}{l}0.892 \\
0.230\end{array}$ \\
\hline 8.0 & $\frac{1}{2}$ & $\begin{array}{l}6.844 \\
5.898\end{array}$ & $\begin{array}{l}5.081 \\
4.806\end{array}$ & $\begin{array}{l}1.228 \\
0.789\end{array}$ & $\begin{array}{l}0.244 \\
0.164\end{array}$ \\
\hline 8.5 & $\frac{1}{2}$ & 5.366 & 4.781 & 0.585 & 0.122 \\
\hline 9.0 & $\frac{1}{2}$ & 8.086 & 4.248 & 0.838 & 0.197 \\
\hline 10.0 & $\frac{1}{2}$ & 5.258 & 4.945 & 0.807 & 0.048 \\
\hline 11.5 & $\frac{1}{2}$ & 4.862 & 4,899 & 0.563 & 0.131 \\
\hline
\end{tabular}




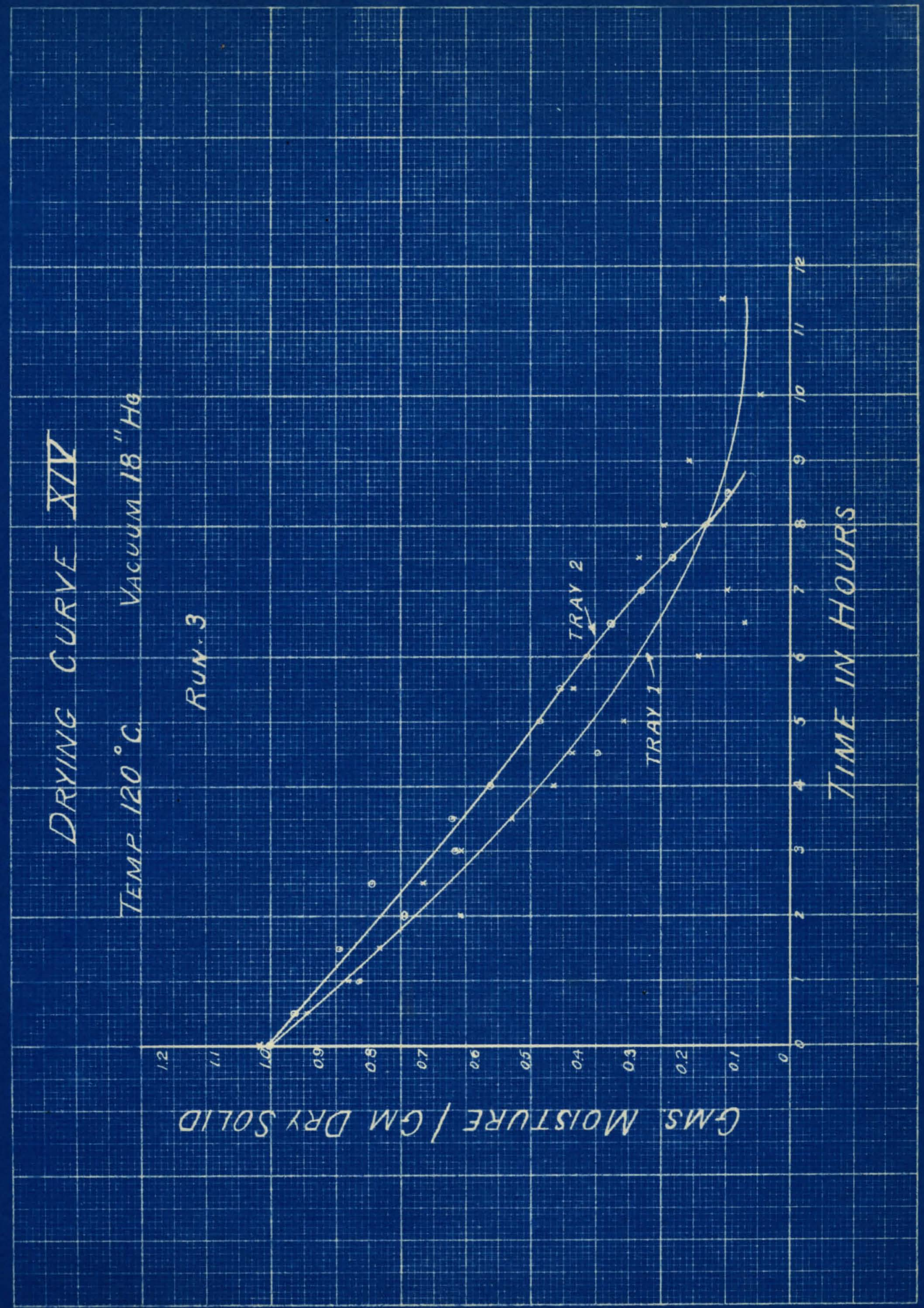


TABLE XXV

LOO SHEEY

Run 4

Department of Chomical Englnoering

Spood Solent1r10 Sohool

Univeralty of Loulaville

\section{DRTIMO OF PRUSSIA BLUE}

Date: $5 / 9 / 34$

Temperature: $130^{\circ} \mathrm{C}$.

Vaculua: $10^{\text {n }}$ Hg.

Plant Reoord Data

rame: Steel Blue

Rx. No.t 4006

S11p 110.: 731

Bat on Ho.: 4

Date struok: $3 / 29 / 34$

No. Tray Driod: 5

Date In: $4 / 30 / 34$
Laboratory Data

Bateh Rod.: $4 / 30 / 34$

Batoh Rtd.: 5/12/34

Ave. Control Temp: $130.55^{\circ} \mathrm{C}$.

Tray Data

Tray Wt. of Cake
Ho. Before Drying
Ibs.

Wt, of Cake

After Drying

1bes.

Wt. Moisture

Lost

Ibe.

$\frac{1}{8}$

6.25
5.50
11.16

3.00

2.75

3.25

$\frac{2.75}{6.00}$ 
PABLE XXVI

CAXS TELPERATRE DN

Run 4

Timo

Brs.

0.85

0.80

0.75

1.00

1.88

1.50

1.75

2.00

8.85

2.80

8.75

3.00

3.85

3.80

3.78

4.00

4.25

4.50

4.75

5.00

6.96

5.60

5.75

6.00

6.85

6.80

6.76

7.00

7.85

7.60

7.76

8.00 may 1 Tempernture in ${ }^{\circ} \mathrm{C}$.

\#2 \#3 $\operatorname{TH}_{4}$ Arg. \#6 $\begin{gathered}\text { Try } 2 \\ \# 6\end{gathered}$ Arg.

$\begin{array}{llllllll}37.0 & 36.0 & 39.0 & 37.3 & 44.0 & 49.5 & 76.5 \% & 46.7\end{array}$

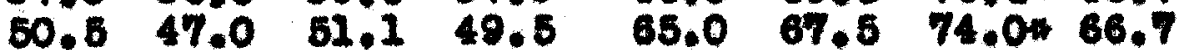

$\begin{array}{llllllll}72.0 & 69.5 & 74.0 & 71.8 & 74.0 & 74.0 & 74.8 & 74.8\end{array}$

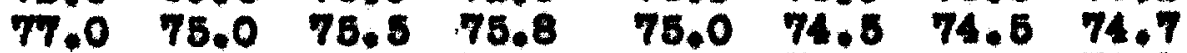

$78.076 .0 \quad 75.5 \quad 76.5$

$76.075 .075 .5 \quad 74.8$

$77.076 .5 \quad 75.076 .8$

7.076 .074 .075 .7

74.875 .5

74.8

74.8

74.574 .075 .574 .0

$75.073 .5 \quad 72.5 \quad 73.7$

$\begin{array}{llll}76.0 & 74.5 & 74.0 & 74.0 \\ 76.0 & 75.5 & 75.0 & 75.8\end{array}$

76.074 .5

72.5

74.3

$78.0 \quad 76.8 \quad 76.0 \quad 76.8$

$76.0 \quad 75.5 \quad 74.0 \quad 74.5$

74.5

730

74.073 .8

$74.0 \quad 72.0 \quad 74.0 \quad 73.3$

$74.0 \quad 72.5 \quad 74.0 \quad 73.5$

$75.0 \quad 73.0 \quad 73.5 \quad 75.8$

$78.074 .5 \quad 74.0 \quad 74.5$

$75.0 \quad 74.5 \quad 74.0 \quad 74.5$

75.0

74.5

74.074 .8

76.5

75.5

75.5

70.0

70.5

74.5

73.5

75.8

78.5

75.6

74.0

73.7

$70.072 .5 \quad 72.3$

$72.0 \quad 73.5 \quad 72.5 \quad 72.7$

$\begin{array}{llll}74.0 & 73.5 & 73.5 & 73.7\end{array}$

$74.0 \quad 75.0 \quad 74.0 \quad 74.5$

$74.0 \quad 75.0 \quad 74.0 \quad 74.5$

$\begin{array}{llll}74.0 & 75.0 & 78.5 & 75.8\end{array}$

$74.0 \quad 74.5 \quad 74.0 \quad 74.2$

$\begin{array}{llll}75.5 & 76.5 & 89.5 & 80.5\end{array}$

$75.0 \quad 74.5 \quad 74.0 \quad 74.5$

$78.0 \quad 78.5 \quad 100.0 \quad 85.6$

$75.0 \quad 74.5 \quad 74.0 \quad 74.2$

$91.0 \quad 84.5112 .5 \quad 96.0$

$74.6 \quad 74.0 \quad 74.2$

108.598 .5182 .5108 .8

$75.0 \quad 74.5 \quad 74.0 \quad 74.2$

209.0120 .5130 .0184 .8

$75.0 \quad 74.5 \quad 74.0 \quad 74.5$

$130 \times 0130.0 \quad 250.0130 .0$

$75.0 \quad 74.5 \quad 81.0 \quad 76.8$

$138.0131 .0 \quad 138.0 \quad 131.7$

$75.0 \quad 74.5 \quad 88.0 \quad 79.2$

$\begin{array}{lllll}78.0 & 75.0 & 98.0 & 85.7\end{array}$

138.0130 .0140 .0138 .0

138.0239 .0140 .0139 .0

$\begin{array}{lllllll}8.5 & 75.0 & 105.5 & 86.0 & 138.0 & 139.0 & 140.0 \quad 139.0\end{array}$

$04.5 \quad 94.5113 .0 \quad 100.7 \quad 135.0 \quad 134.0 \quad 134.0 \quad 134.3$

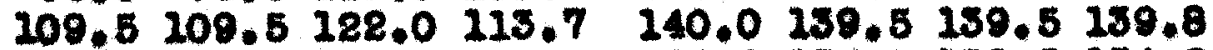

117.8117 .5123 .5119 .5

$134.0136 .0132,5134.8$

$136.0 \quad 235.5 \quad 135.5135 .7$

- Valuo onlttod in evorago. 


\section{CARE TEMRERATURE CURVE TI}

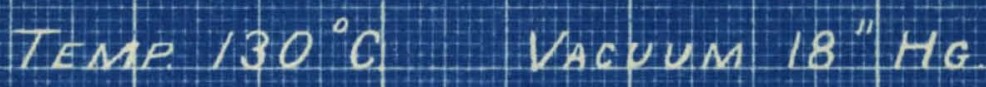

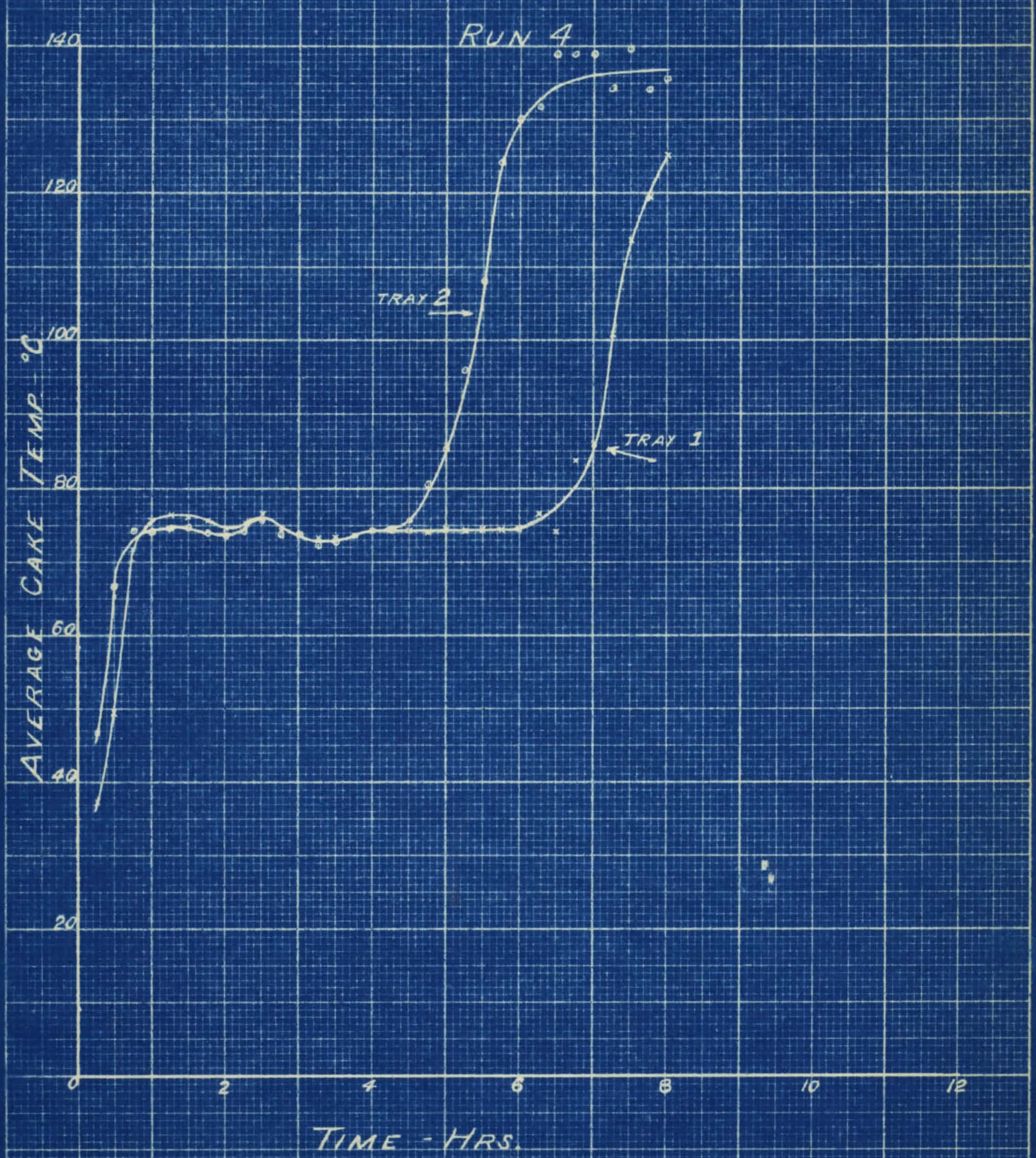


THBLE XXVII

MOISTURE CONTENT DATA

Run 4

Time Tray wt, Wot

Hirs. Ho. Samplo

Gins.

0

$\begin{array}{ll}1 & 4.066 \\ 2 & 4.877\end{array}$

0.5

$\frac{1}{2}$

5.336
4.726

$1.0 \quad \frac{1}{2}$

6,248

6,544

1,5

1

5,665

6.074

wt

Semple

Oms.

3.108
2.483

3.838

2.619

3.388

3,885

5.714

4.677

3,140

2.673

4,865
4.375

2,871

3.241

8.843

2.711

3.043

2.154

1.332

3.202

5.476
5.186

4.596

$2 \cdot 274$

0.590

$\begin{array}{ll}5.310 & 3.288 \\ 4.010 & 3.365\end{array}$

2.022

0.645

3.485

5.507
5.713

5.181

2.028

0.552

3.653

5.537
4.287

3.920

1.884

$4.175 \quad 2.928$

4.204

0.042

$5.411 \quad 1.676$

5.527

0.042

4.859

5.086

1.351

0.007

0.292

0.443

0.794

0.458

0.710

0.128

0.614

0.192

0.580

0.103

0.517

0.094

0.460

0.010

0.310

0.008

$\begin{array}{llllll}6.0 & 1 & 6.210 & 4.859 & 1.351 & 0.278 \\ & 2 & 5.093 & 5.086 & 0.007 & 0.001\end{array}$




\section{TABLE XXVII (Cent.)}

HOI8HORE CONTENT DAM

Run 4

\begin{tabular}{|c|c|c|c|c|c|}
\hline Fre. & $\begin{array}{c}\text { Tras } \\
\text { Ho. }\end{array}$ & $\begin{array}{c}\text { wt. Wet } \\
\text { simplo } \\
\text { oms. }\end{array}$ & $\begin{array}{l}\text { Wt. Dir. } \\
\text { simplo } \\
\text { Cans. }\end{array}$ & $\begin{array}{l}\text { Moloture } \\
\text { Iost } \\
\text { Onde. }\end{array}$ & $\begin{array}{l}\text { One. Holat } \\
\text { por Ger } \\
\text { Dery solla }\end{array}$ \\
\hline 6.5 & $\begin{array}{l}1 \\
2\end{array}$ & $\begin{array}{l}4.547 \\
3.605\end{array}$ & $\begin{array}{l}3.674 \\
3.598\end{array}$ & $\begin{array}{l}0.875 \\
0.010\end{array}$ & $\begin{array}{l}0.238 \\
0.003\end{array}$ \\
\hline 7.0 & $\frac{1}{8}$ & $\begin{array}{l}6.066 \\
3.708\end{array}$ & $\frac{5.152}{3.703}$ & $\begin{array}{l}0.014 \\
0.008\end{array}$ & $\begin{array}{l}0.178 \\
0.001\end{array}$ \\
\hline 7.5 & $\begin{array}{l}2 \\
2\end{array}$ & 4.818 & 3.873 & 0,045 & 0.848 \\
\hline 8.0 & $\begin{array}{l}1 \\
8\end{array}$ & $\begin{array}{l}5.565 \\
4.435\end{array}$ & $\begin{array}{l}6.589 \\
4.448\end{array}$ & 0.006 & 0.001 \\
\hline
\end{tabular}




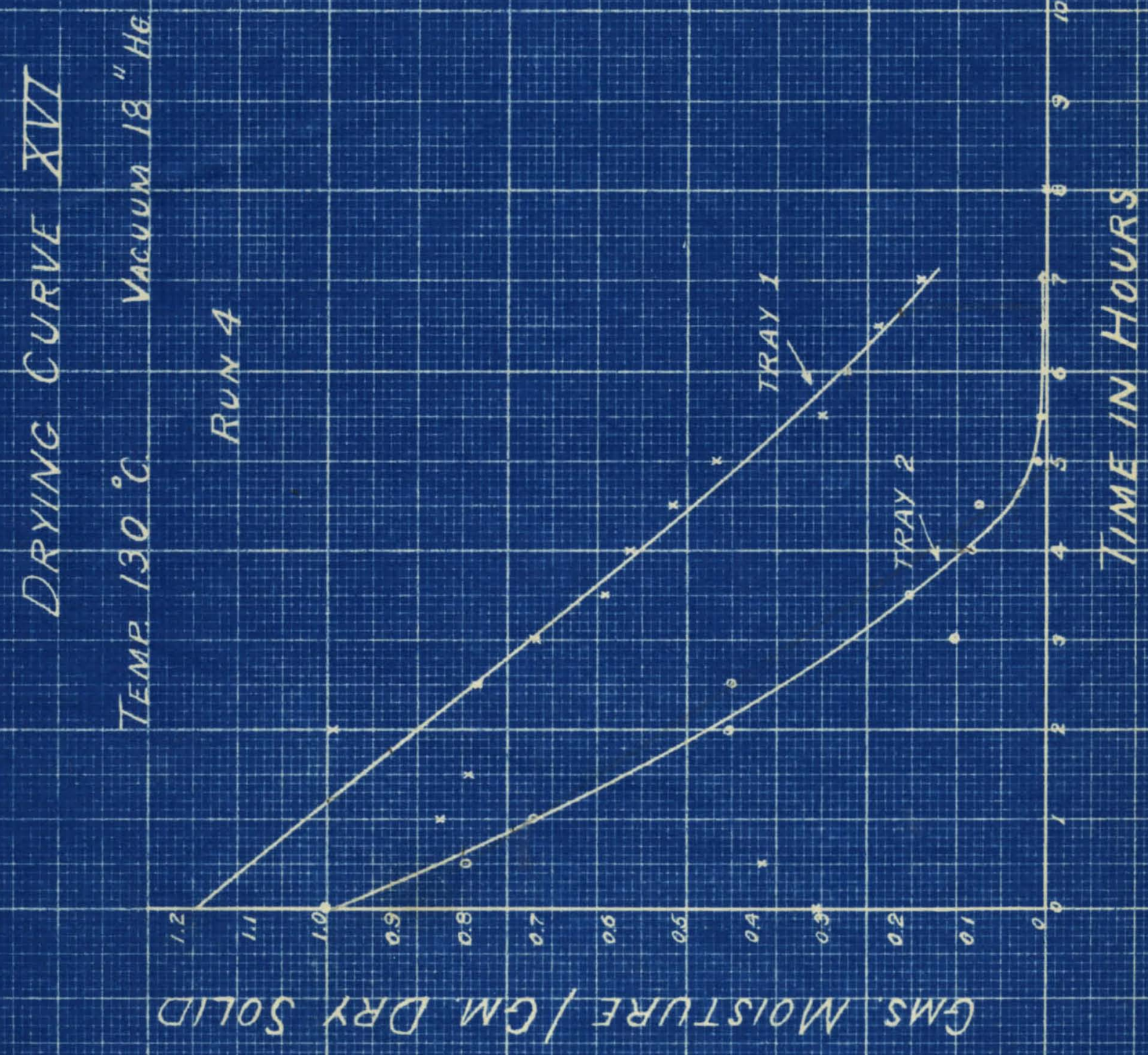




\section{TABIE XXVIII}

LOG SHEET

Run 5

Department of Chemlcal Englneering

Speed Scientifle School

Un1versity of Louisville

\section{DRYING OF PRUSSIAN BLUE}

Date: $5 / 5 / 34$

Temperature: $100^{\circ} \mathrm{C}$.

Plant Record Data

Nane: Steel Blue

Px. No.: 4006

S1Ip No.: 731

Batch No.: \&

Date Striek: $3 / 29 / 34$

No. Trays Dried: 5

Date In: $4 / 30 / 34$
Vacuum: $22^{n} \mathrm{Hg}$.
Batch Red.: $4 / 30 / 34$

Batch Rtd.: $5 / 12 / 34$

Avg. Control Temp: $100.55^{\circ} \mathrm{C}$.

Tray Data

Tray wt. of Cake

No. Before Drying $1 \mathrm{bs}$.



wt. of Gake

After Drying 1 bs.

3.00

$\frac{3.25}{6.25}$
Wt. Molsture

Lost

Ibs.

3.75

$\frac{3.50}{7.25}$ 
TABIE XXIX

CAKE TEMPERA TURE DATA

Run 5

Timo

Exre.

0.25

0.50

0.75

1.00

1.25

1.50

1.75

2.00

2.25

2.50

2.75

3.00

3.25

3.80

3.75

4.00

4.25

4.50

4.75

5.00

5.25

5.50

5.75

6.00

6.25

6.50

6.75

7.00

7.85

7.60

7.75

8.00

8.25

8.50

8.75

9.00
Trag 1

Tomperatures in ${ }^{\circ} \mathrm{C}$

\section{i.} \#0 AV8.

\section{$65.0 \quad 54.5$}

65.564 .5

64.0

62.0

64.5

63.0

62.0

68.0

64.5

62.5

51.0

63.0

53.5

$64.0 \quad 64.2$

61.061 .7

$62,062.5$

61.5

61.0

61.5

64.0

62.5

64.0

63.5

65.0

62.5

62.0

62.8

64.5

64.5

64.5

64.7

65.5

63.5

63.0

63.0

64.8

64.5

63.7

64.8

65.0

68.5

62.0

65.5

63.5

65.0

62.5

63.8

63.5

63.5

62.5

63.2

66.0

62.5

63.0

65.0

64.5

64.5

65.5

65.5

64.5

66.0

65.5

65.0

65.5

65.0

64.5

65.0

65.5

65.5

65.0

66.0

65.0

65.0

65.5

65.5

65.0

65.0

66.0

65.5

65.0

65.5

65.0

65.0

64.5

64.5

65.5

65.0

64.0

65.0

66.0

65.5

62.0

64.0

64.0

65.5

65.0

64.0

65.0

65.5

67.0

66.5

66.0

67.0

63.5

63.0

63.0

65.0

65.0

65.3

65.2

65.3

65.2

65.0

65.8

65.5

65.0

65.0

65.0

65.5

63.5

65.0

64.8

66.5

64.5

66.0

63.5

62.5

64.0

62.0

63.5

61.0

62.2

62.3
$\# \operatorname{Tag}^{26} 2$

45.0

58.0

64.0

62.0

61.0

60.0

62.0

62.0

64.0

64.0

64.5

62.5

63.5

63.5

63.5

65.0

64.0

64.5

64.5

65.5

65.0

65.0

65.0

65.0

65.0

65.0

64.0

65.0

65.0

65.0

63.0

66.0

63.0

62.0

61.0

60.5

\section{5}

$56.5 \quad 59.5 \quad 58.0$

$63.5 \quad 63.5 \quad 63.7$

$59.5 \quad 60.5 \quad 60.7$

$59.0 \quad 60.0 \quad 60.0$

$58.5 \quad 59.0 \quad 59.2$

$65.5 \quad 64.5 \quad 64.0$

$61.5 \quad 61.7 \quad 61.7$

$64.5 \quad 65.5 \quad 64.0$

$62.5 \quad 62.5 \quad 63.0$

$64.0 \quad 63.5 \quad 64.0$

$61.5 \quad 61.5 \quad 61.8$

$63.0 \quad 63.5 \quad 63.3$

$62.0 \quad 62.0 \quad 62.5$

$63.5 \quad 65.5 \quad 63.5$

$64.5 \quad 64.5 \quad 64.8$

$64.0 \quad 64.5 \quad 64.2$

$65.5 \quad 64.0 \quad 64.7$

$64.5 \quad 64.5 \quad 64.5$

$64.0 \quad 64.0 \quad 64.5$

$64.5 \quad 64.0 \quad 64.5$

$65.0 \quad 64.5 \quad 65.0$

$64.5 \quad 64.064 .5$

$65.0 \quad 64.5 \quad 6498$

$64.8 \quad 64.0 \quad 64.5$

$64.5 \quad 64.5 \quad 64.7$

$64.5 \quad 64.5 \quad 64.5$

$64.0 \quad 65.5 \quad 64.8$

$64.5 \quad 65.5 \quad 65.0$

$64.5 \quad 65.5 \quad 65.0$

63.564 .563 .7

$65.5 \quad 6 B .5 \quad 65.7$

$62.0 \quad 61.0 \quad 62.0$

$61.5 \quad 61.5 \quad 61.7$

$60.5 \quad 60.5 \quad 60.7$

$61.0 \quad 61.0 \quad 60.8$ 


\section{TABLE XXIX (Cont.)}

\section{CA KE TEMPERA TOEE DA TA}

fun 5

Time

Exs.

9.28

9.80

9.75

10.00

10.28

10.50

10.75

11.00

11.26

12. 50

11.75

18.00
48 Tray 1 $\operatorname{twat}^{2} 1$

Temperaturea $\ln { }^{\circ} \mathrm{C}$. \#4 A78. \#5 \#6 \#7 AVB.

$$
\begin{array}{llll}
63.0 & 62.5 & 61.0 & 62.2
\end{array}
$$

$64.5 \quad 63.5 \quad 68.0 \quad 63.3$

61.5

61.5 6.062 .5

64.062 .5

64.068 .5

65.065 .5

64.064 .5

65.067 .5

65.074 .0

67.076 .0

66.081 .0

$70.0 \quad 87.0$

70.0

01.0

$\begin{array}{ll}62.0 & 61.7 \\ 62.5 & 68.7 \\ 63.5 & 63.5 \\ 64.5 & 63.7 \\ 65.5 & 65.5 \\ 65.5 & 64.7 \\ 69.5 & 67.4 \\ 75.5 & 70.8 \\ 7655 & 72.8 \\ 61.0 & 75.7 \\ 88.0 & 81.7 \\ 89.5 & 83.8\end{array}$


CAkE tEMPERATURE CURVE TIII

TEMP 100 O. VacuUm $22^{\circ} \mathrm{He}$

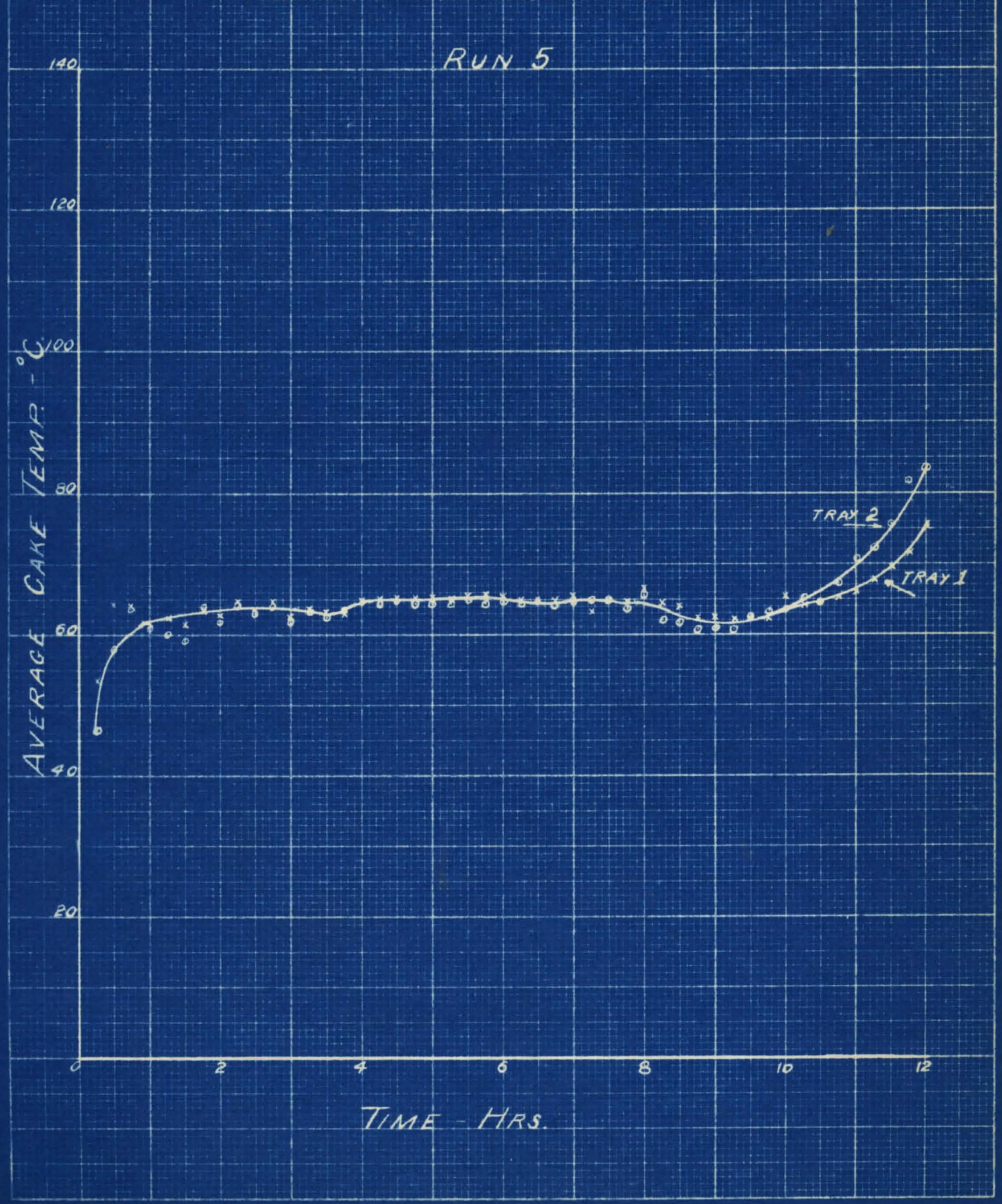


TABLS $\mathbf{X X X}$

MOISTURE CONTENT DATA

Run B

\begin{tabular}{|c|c|c|c|c|c|}
\hline Time & $\begin{array}{c}\text { Pray } \\
\text { No. }\end{array}$ & $\begin{array}{l}\text { Wt. Wet } \\
\text { samplo } \\
\text { Gme. }\end{array}$ & $\begin{array}{c}\text { Wt. Dry } \\
\text { Samp } 10 \\
\text { Cns. }\end{array}$ & $\begin{array}{l}\text { Moleture } \\
\text { Iost } \\
\text { Cons. }\end{array}$ & $\begin{array}{c}\text { Onw Mo1st } \\
\text { por Can; } \\
\text { Dry Solid }\end{array}$ \\
\hline 0 & $\frac{1}{2}$ & $\begin{array}{l}3.445 \\
4.488\end{array}$ & $\begin{array}{l}1.743 \\
2.250\end{array}$ & $\begin{array}{l}1.702 \\
8.178\end{array}$ & $\begin{array}{l}0.875 \\
0.969\end{array}$ \\
\hline 0.5 & $\frac{1}{8}$ & $\begin{array}{l}5.177 \\
3.628\end{array}$ & $\begin{array}{l}2.485 \\
1.922\end{array}$ & $\begin{array}{l}2.762 \\
1.716\end{array}$ & $\begin{array}{l}1.233 \\
0.897\end{array}$ \\
\hline 1.0 & $\frac{1}{2}$ & $\begin{array}{l}4.755 \\
4.359\end{array}$ & $\begin{array}{l}8.308 \\
2.558\end{array}$ & $\begin{array}{l}8.447 \\
1.981\end{array}$ & $\begin{array}{l}0.061 \\
0.774\end{array}$ \\
\hline 1.5 & $\frac{1}{2}$ & $\begin{array}{l}5.296 \\
4.013\end{array}$ & $\begin{array}{l}2.786 \\
2.825\end{array}$ & $\begin{array}{l}2.510 \\
8.100\end{array}$ & $\begin{array}{l}0.000 \\
0.746\end{array}$ \\
\hline 2.0 & $\begin{array}{l}1 \\
2\end{array}$ & $\begin{array}{l}6.651 \\
4.960\end{array}$ & $\begin{array}{l}3.419 \\
2.950\end{array}$ & $\begin{array}{l}3.212 \\
8.010\end{array}$ & $\begin{array}{l}0.989 \\
0.682\end{array}$ \\
\hline 2.8 & $\frac{1}{2}$ & $\begin{array}{l}3.655 \\
5.314\end{array}$ & $\begin{array}{r}2.056 \\
3.066\end{array}$ & $\begin{array}{l}1.598 \\
8.848\end{array}$ & $\begin{array}{l}0.778 \\
0.735\end{array}$ \\
\hline 3.0 & $\frac{1}{2}$ & $\begin{array}{l}5.164 \\
6.521\end{array}$ & $\begin{array}{l}3.068 \\
3.887\end{array}$ & $\begin{array}{l}8.106 \\
2.634\end{array}$ & $\begin{array}{l}0.688 \\
0.677\end{array}$ \\
\hline 3.6 & $\frac{1}{2}$ & $\begin{array}{l}6.084 \\
6.771\end{array}$ & $\begin{array}{l}3.436 \\
4.177\end{array}$ & $\begin{array}{l}2.648 \\
2.594\end{array}$ & $\begin{array}{l}0.771 \\
0.622\end{array}$ \\
\hline 4.0 & $\frac{1}{2}$ & $\begin{array}{l}6.572 \\
6.596\end{array}$ & $\begin{array}{l}3.869 \\
4.308\end{array}$ & $\begin{array}{l}8.703 \\
2.290\end{array}$ & $\begin{array}{l}0.698 \\
0.800\end{array}$ \\
\hline 4.5 & $\frac{1}{2}$ & $\begin{array}{l}4.618 \\
6.388\end{array}$ & $\begin{array}{l}2.975 \\
4.605\end{array}$ & $\begin{array}{l}2.608 \\
2.303\end{array}$ & $\begin{array}{l}0.563 \\
9.518\end{array}$ \\
\hline 5.0 & $\frac{1}{8}$ & $\begin{array}{l}6.588 \\
6.612\end{array}$ & $\begin{array}{r}4.178 \\
4.376\end{array}$ & $\begin{array}{l}2.217 \\
2.256\end{array}$ & $\begin{array}{l}0.531 \\
0.512\end{array}$ \\
\hline 5.5 & $\begin{array}{l}1 \\
2\end{array}$ & $\begin{array}{l}6.380 \\
8.077\end{array}$ & $\begin{array}{l}4.055 \\
3.758\end{array}$ & $\begin{array}{l}2.325 \\
1.359\end{array}$ & $\begin{array}{l}0.573 \\
0.368\end{array}$ \\
\hline 6.0 & $\frac{1}{2}$ & $\begin{array}{l}6.281 \\
5.205\end{array}$ & $\begin{array}{l}4.086 \\
3.855\end{array}$ & $\begin{array}{l}2.185 \\
1.358\end{array}$ & $\begin{array}{l}0.587 \\
0.381\end{array}$ \\
\hline
\end{tabular}


TABLs XXX (cont.)

MOISFUFE CONTEWT DATA

Run 5

\begin{tabular}{|c|c|c|c|c|c|}
\hline $\begin{array}{l}\text { Timo } \\
\text { Fre. }\end{array}$ & $\begin{array}{c}\text { Tray } \\
\text { No. }\end{array}$ & $\begin{array}{l}\text { Wt. Wot } \\
\text { simple } \\
\text { Cone. }\end{array}$ & $\begin{array}{c}\text { Wt. Dury } \\
\text { semple } \\
\text { Oms. }\end{array}$ & $\begin{array}{c}\text { Moleture } \\
\text { Imost } \\
\text { Gane. }\end{array}$ & $\begin{array}{c}\text { Cone. Molet } \\
\text { per Cant solid }\end{array}$ \\
\hline 6.5 & $\frac{1}{2}$ & $\begin{array}{l}6.085 \\
6.828\end{array}$ & $\begin{array}{l}3.925 \\
5.285\end{array}$ & $\begin{array}{l}2.160 \\
1.605\end{array}$ & $\begin{array}{l}0.589 \\
0.307\end{array}$ \\
\hline 7.0 & $\frac{1}{2}$ & $\begin{array}{l}4.786 \\
8.380\end{array}$ & $\begin{array}{l}3.446 \\
4.475\end{array}$ & $\begin{array}{l}1.880 \\
0.816\end{array}$ & $\begin{array}{l}0.371 \\
0.806\end{array}$ \\
\hline 7.5 & $\frac{1}{8}$ & $\begin{array}{l}6.558 \\
4.781\end{array}$ & $\begin{array}{l}4.857 \\
4.066\end{array}$ & $\begin{array}{l}8.175 \\
0.685\end{array}$ & $\begin{array}{l}0.490 \\
0.261\end{array}$ \\
\hline 8.0 & $\frac{2}{2}$ & $\begin{array}{l}5.198 \\
4.470\end{array}$ & $\begin{array}{l}1.065 \\
3.761\end{array}$ & $\begin{array}{l}1.280 \\
0.700\end{array}$ & $\begin{array}{l}0.279 \\
0.189\end{array}$ \\
\hline 8.6 & $\begin{array}{l}1 \\
8\end{array}$ & $\begin{array}{l}6.742 \\
4.662\end{array}$ & $\begin{array}{l}4.206 \\
4.000\end{array}$ & $\begin{array}{l}0.656 \\
0.685\end{array}$ & $\begin{array}{l}0.388 \\
0.138\end{array}$ \\
\hline 9.0 & $\frac{1}{2}$ & $\begin{array}{l}3.874 \\
4.301\end{array}$ & $\begin{array}{l}3.290 \\
4.214\end{array}$ & $\begin{array}{l}0.888 \\
0.267\end{array}$ & $\begin{array}{l}0.178 \\
0.040\end{array}$ \\
\hline 8.6 & $\frac{1}{2}$ & $\begin{array}{l}4.810 \\
5.345\end{array}$ & $\begin{array}{l}3.066 \\
4.780\end{array}$ & $\begin{array}{l}0.544 \\
0.565\end{array}$ & $\begin{array}{l}0.137 \\
0.118\end{array}$ \\
\hline 10.0 & $\frac{1}{2}$ & $\begin{array}{r}4.828 \\
4.468\end{array}$ & $\begin{array}{l}4.128 \\
4.020\end{array}$ & $\begin{array}{l}0.400 \\
0.448\end{array}$ & $\begin{array}{l}0.097 \\
0.111\end{array}$ \\
\hline 10.8 & $\frac{1}{2}$ & $\begin{array}{r}5.898 \\
5.528\end{array}$ & $\begin{array}{l}4.785 \\
4.464\end{array}$ & $\begin{array}{l}1.123 \\
0.044\end{array}$ & $\begin{array}{l}0.825 \\
0.010\end{array}$ \\
\hline 11.0 & $\frac{1}{2}$ & $\begin{array}{l}5.141 \\
6.049\end{array}$ & $\begin{array}{l}5.118 \\
4.725\end{array}$ & $\begin{array}{l}0.023 \\
0.526\end{array}$ & $\begin{array}{l}0.004 \\
0.060\end{array}$ \\
\hline 11.5 & $\frac{1}{2}$ & $\begin{array}{l}4.998 \\
4.709\end{array}$ & $\begin{array}{l}4.996 \\
4.616\end{array}$ & $\begin{array}{l}0.008 \\
0.175\end{array}$ & $\begin{array}{l}0.000 \\
0.087\end{array}$ \\
\hline 18.0 & $\frac{1}{2}$ & $\begin{array}{l}4.697 \\
6.057\end{array}$ & $\begin{array}{l}4.870 \\
6.003\end{array}$ & $\begin{array}{l}0.127 \\
0.054\end{array}$ & $\begin{array}{l}0.088 \\
0.008\end{array}$ \\
\hline
\end{tabular}




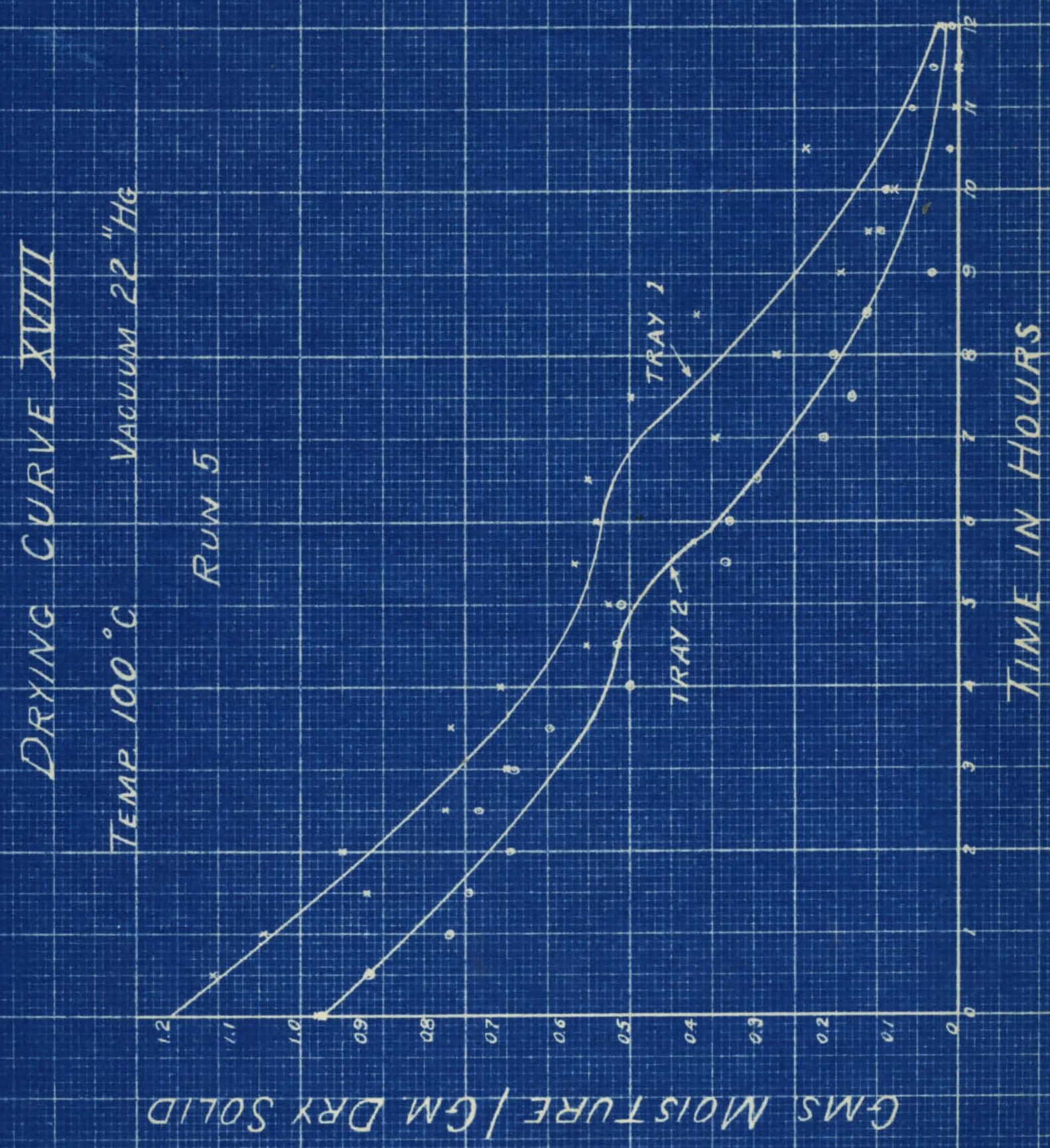


TABLE XXXI

LOO SHBET

Run 6

Department of Chomical Englnoering

Spoed Solentifio Sohool University of Loulavilio

DRYINO OF PROSSIAX BLUR

Date: $5 / 7 / 34$

Temperature: $110^{\circ} \mathrm{C}$.

Vacuum: $22^{*} \mathrm{Hg}$.

Plant Record Date

Laboratory Deta

Name: Steel Blue

Rx. No.: 4006

SIIp No.: 731

Batch No.: 4

Date struck: $3 / 29 / 34$

No. Trays Dried: 5

Date In: $4 / 30 / 34$

Batoh Rod.: 4/30/34

Batch Rtd.: $5 / 12 / 34$

Avg. Control Temp $110.54^{\circ} \mathrm{C}$.

Tray Data

\begin{tabular}{|c|c|c|c|c|}
\hline $\begin{array}{c}\text { Tray } \\
\text { No. }\end{array}$ & $\begin{array}{l}\text { Wt. of Cake } \\
\text { Before Dry } 2 \text { se } \\
\text { IDE. }\end{array}$ & $\begin{array}{l}\text { Wt. of Cake } \\
\text { After Dry Ins } \\
\text { Ibs. }\end{array}$ & wt. & $\begin{array}{l}\text { Molsture } \\
\text { Lost } \\
\text { Ibs. }\end{array}$ \\
\hline 1 & $\begin{array}{r}6.25 \\
6.00 \\
12.26\end{array}$ & $\begin{array}{l}2.50 \\
2.75 \\
5.25\end{array}$ & & $\begin{array}{r}3.75 \\
3.25 \\
7.00\end{array}$ \\
\hline
\end{tabular}


TABLE XXXII

CAKE TEMPERA TURE DATA

Run 6

Time

Fre.

0.25

0.50

0.75

1.00

1.25

1.50

1.75

2.00

2.25

2.80

2.75

3.00

3.25

3.50

3.75

4.00

4.25

4.50

4.75

5.00

5.25

5.50

5.75

6.00

6.25

6.50

6.75

7.00

7.25

7.50

7.75

8.00

8.25

8. 50

0.75

9.00
Trag 1

\#8

Temperaturea in ${ }^{\circ} \mathrm{C}$

$\# 3$ \#4 Avg. \#5 \#6 \#7 Avg.

$63.0 \% 35.5 \quad 37.0 \quad 36.3$

$70.0 * 53.0$

82.0\% 64.0

56.0

65.0

54.5

64.5

65.0

86.5 65.0

66.065 .5

65.0

66.8

66.0

66.0

65.5

65.5

65.

66.0

65.5

65.0

65.0

65.0

66.0

65.5

65.5

65.0

66.0

65.0

65.0

65.5

65.5

65.5

65.5

66.0

66.0

66.0

66.0

66.0

66.0

66.5

66.0

68.5

68.0

68.0

68.0

68.0

68.0

68.0

67.5

68.0

68.0

68.5

68.0
65.0

65.

64.5

64.5

64.5

66.0

66.0

65.0

65.5

66.5

65.5

67.5

67.0

67.5

67.5

67.5

65.5

67.5

67.0

67.5

67.5

67.5

65.5
65.3

65.0

65.2

65.3

65.2

65.3

65.2

65.2

66.0

66.0

65.5

65.5

66.7

65.8

67.8

67.3

67.7

67.7

67.5

66.5

67.5

67.0

67.7

70.5

67.8

66.8 $\begin{array}{llll}4.0 & 43.5 & 41.5 & 43.0\end{array}$

$53.0 \quad 61.5 \quad 61.5^{\prime} 58.7$

$65.0 \quad 64.5 \quad 65.6 \quad 65.6$

$\begin{array}{llll}65.0 & 65.5 & 65.5 & 65.3\end{array}$

$65.0 \quad 65.0 \quad 65.5 \quad 65.2$

$65.0 \quad 65.0 \quad 65.5 \quad 65.2$

$65.0 \quad 65.0 \quad 65.0 \quad 65.0$

$65.0 \quad 65.0 \quad 65.0 \quad 65.0$

$65.0 \quad 65.0 \quad 65.0 \quad 65.0$

$65.0 \quad 65.0 \quad 65.0 \quad 65.0$

$65.0 \quad 65.0 \quad 65.0 \quad 65.0$

$65.0 \quad 65.0 \quad 65.0 \quad 65.0$

$65.0 \quad 65.0 \quad 65.0 \quad 65.0$

$65.0 \quad 65.0 \quad 65.0 \quad 65.0$

$\begin{array}{llll}65.0 & 65.5 & 65.0 & 65.2\end{array}$

$65.5 \quad 66.0 \quad 65.0 \quad 65.5$

$66.0 \quad 65.0 \quad 65.0 \quad 65.3$

$66.0 \quad 65.0 \quad 65.0 \quad 65.3$

$66.0 \quad 66.5 \quad 66.0 \quad 66.2$

$66.0 \quad 66.5 \quad 66.0 \quad 66.2$

$66.0 \quad 66.0 \quad 65.5 \quad 65.8$

$67.0 \quad 66.5 \quad 66.0 \quad 66.5$

$66.5 \quad 67.0 \quad 66.5 \quad 66.7$

$66.5 \quad 66.0 \quad 66.0 \quad 66.8$

$68.0 \quad 67.5 \quad 68.0 \quad 67.3$

$\begin{array}{llll}67.0 & 67.5 & 67.0 & 67.2\end{array}$

$67.0 \quad 67.0 \quad 67.0 \quad 67.0$

$67.0 \quad 66.0 \quad 66.0 \quad 66.3$

$\begin{array}{lllll}68.0 & 69.0 & 66.5 & 67.8\end{array}$

$68.0 \quad 69.5 \quad 67.5 \quad 68.3$

$68.0 \quad 78.0 \quad 67.0 \quad 69.0$

$\begin{array}{llll}68.5 & 78.5 & 70.5 & 72.5\end{array}$

$\begin{array}{llll}70.0 & 83.5 & 83.5 & 79.0\end{array}$

70.5

88.5

83.5

80.8

78.0

93.0

89.5

84.0

$\begin{array}{lllll}78.0 & 99.5 & 97.5 & 91.7\end{array}$ 
TABIE XXXII (Cont.)

CARE TEMPBRATURE DATA

Aun 6

\begin{tabular}{|c|c|c|c|c|c|c|c|c|}
\hline & & & & & $\mathrm{ln}$ & & & \\
\hline & \#t & 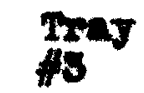 & $\#$ & $A \nabla C$ & $\$ 5$ & & $8_{47}$ & $\nabla \mid$ \\
\hline $\begin{array}{r}9.85 \\
9.50 \\
8.75 \\
10.00 \\
10.25 \\
10.00 \\
10.75 \\
11.00 \\
11.25 \\
11.00\end{array}$ & $\begin{array}{l}66.0 \\
68.0 \\
68.5 \\
64.0 \\
64.0 \\
64.8 \\
64.0 \\
66.0 \\
69.5 \\
72.5\end{array}$ & $\begin{array}{l}66.0 \\
67.0 \\
64.8 \\
62.5 \\
68.5 \\
64.0 \\
62.5 \\
62.5 \\
62.5 \\
64.5\end{array}$ & $\begin{array}{l}67.0 \\
66.5 \\
64.5 \\
64.0 \\
64.5 \\
64.5 \\
65.0 \\
68.0 \\
73.0 \\
79.5 \\
81.5 \\
89.0\end{array}$ & $\begin{array}{l}66.8 \\
67.8 \\
64.8 \\
63.8 \\
64.0 \\
64.8 \\
63.8 \\
68.8 \\
68.3 \\
78.8 \\
73.8\end{array}$ & $\begin{array}{r}87 \\
88 \\
86 \\
201 \\
103 \\
110 \\
105 \\
112 \\
110 \\
118\end{array}$ & & $\begin{array}{l}10 \\
10 \\
10 \\
11 \\
10 \\
11\end{array}$ & $\begin{array}{l}1 \\
1 \\
1\end{array}$ \\
\hline
\end{tabular}


CAKE TEMPEPATURE CURVE XIX

TEME $110^{\circ} \mathrm{C}$ VACUUM $22^{\prime H C}$

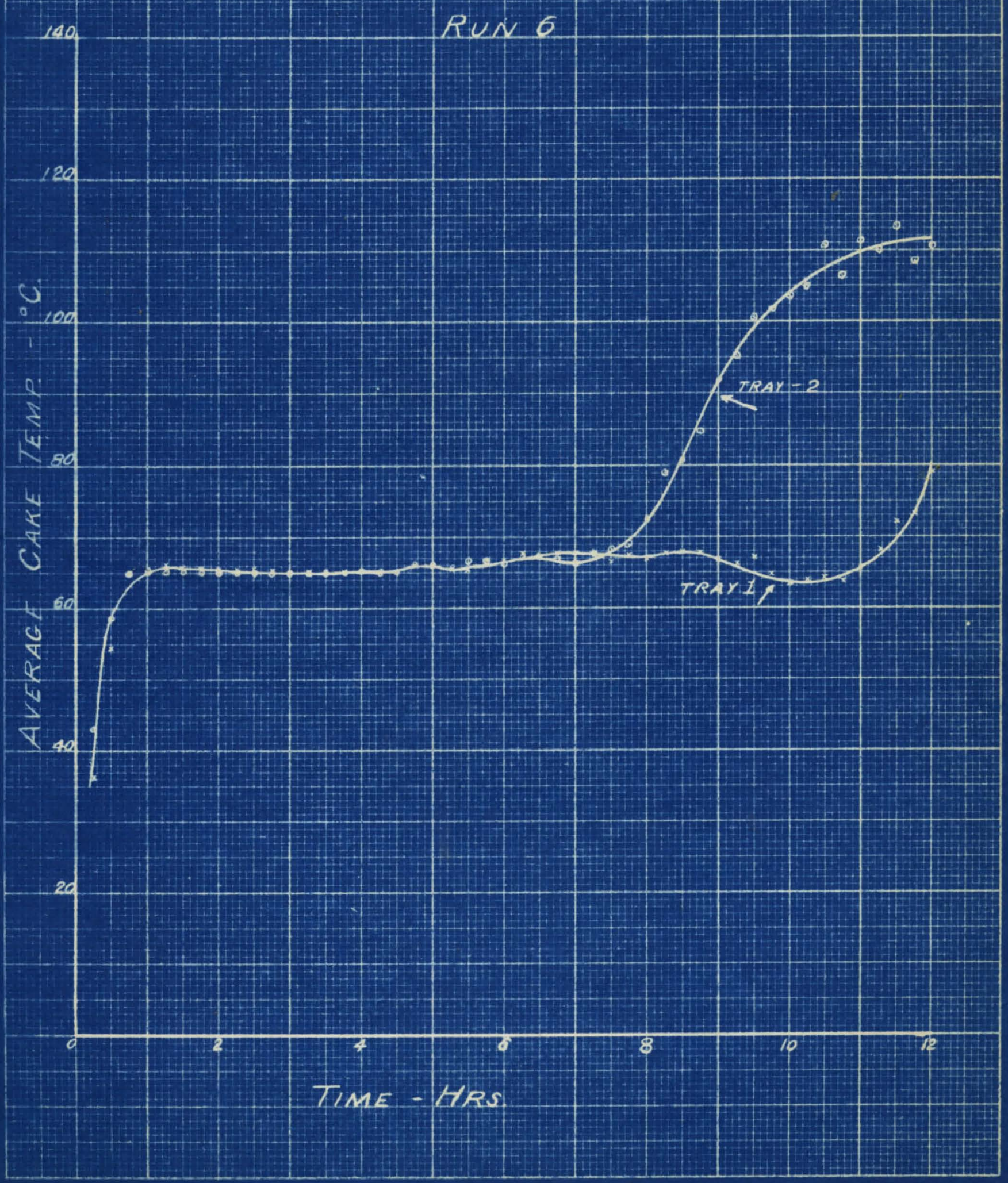




\section{TABLE XXXIII}

\section{MOISTURE CONTENT DATA}

Run 6

\begin{tabular}{|c|c|c|c|c|c|}
\hline $\begin{array}{l}\text { Time } \\
\text { Frs. }\end{array}$ & $\begin{array}{l}\text { Tray w } \\
\text { No. }\end{array}$ & $\begin{array}{c}\text { wt. Wet } \\
\text { Sample } \\
\text { Cms. }\end{array}$ & $\begin{array}{l}\text { wt. Dry } \\
\text { Sample } \\
\text { Gms. }\end{array}$ & $\begin{array}{l}\text { Molsture } \\
\text { Lost } \\
\text { Gms. }\end{array}$ & $\begin{array}{l}\text { Gins. Molst } \\
\text { per Gm. } \\
\text { Dry Solid }\end{array}$ \\
\hline 0 & $\frac{1}{2}$ & $\begin{array}{l}5.704 \\
4.300\end{array}$ & $\begin{array}{l}2.587 \\
2.010\end{array}$ & $\begin{array}{l}3.127 \\
2.290\end{array}$ & $\begin{array}{l}1.202 \\
0.140\end{array}$ \\
\hline 0.5 & $\frac{1}{2}$ & $\begin{array}{l}3.461 \\
3.510\end{array}$ & $\begin{array}{l}1.703 \\
1.792\end{array}$ & $\begin{array}{l}1.758 \\
1.718\end{array}$ & $\begin{array}{l}1.032 \\
0.958\end{array}$ \\
\hline 1.0 & $\frac{1}{2}$ & $\begin{array}{l}5.006 \\
5.702\end{array}$ & $\begin{array}{l}2.545 \\
2.881\end{array}$ & $\begin{array}{l}2.461 \\
2.821\end{array}$ & $\begin{array}{l}0.967 \\
0.979\end{array}$ \\
\hline 2.5 & $\frac{1.012}{2}$ & $\begin{array}{l}6.011 \\
4.566\end{array}$ & $\begin{array}{l}3.141 \\
2.434\end{array}$ & $\begin{array}{l}2.870 \\
2.132\end{array}$ & $\begin{array}{l}0.913 \\
0.875\end{array}$ \\
\hline 2.0 & $\frac{1}{2}$ & $\begin{array}{l}6.025 \\
4.269\end{array}$ & $\begin{array}{l}3.102 \\
2.413\end{array}$ & $\begin{array}{l}2.923 \\
1.865\end{array}$ & $\begin{array}{l}0.943 \\
0.769\end{array}$ \\
\hline 2.5 & $\frac{1}{2}$ & $\begin{array}{l}6.901 \\
3.826\end{array}$ & $\begin{array}{l}3.861 \\
2.304\end{array}$ & $\begin{array}{l}30040 \\
1.522\end{array}$ & $\begin{array}{l}0.787 \\
0.659\end{array}$ \\
\hline 3.0 & $\frac{1}{2}$ & $\begin{array}{l}5.675 \\
4.814\end{array}$ & $\begin{array}{l}3.247 \\
3.033\end{array}$ & $\begin{array}{l}2.438 \\
1.781\end{array}$ & $\begin{array}{l}0.747 \\
0.588\end{array}$ \\
\hline 3.5 & $\frac{1}{2}$ & $\begin{array}{l}7.010 \\
5.340\end{array}$ & $\begin{array}{l}4.099 \\
3.356\end{array}$ & $\begin{array}{l}2.911 \\
1.984\end{array}$ & $\begin{array}{l}0.710 \\
0.592\end{array}$ \\
\hline 4.0 & $\frac{1}{2}$ & $\begin{array}{l}6.864 \\
6.754\end{array}$ & $\begin{array}{l}3.854 \\
4.389\end{array}$ & $\begin{array}{l}3.010 \\
2.355\end{array}$ & $\begin{array}{l}0.782 \\
6.536\end{array}$ \\
\hline 4.5 & $\frac{1}{2}$ & $\begin{array}{l}7.461 \\
6.933\end{array}$ & $\begin{array}{l}4.756 \\
4.504\end{array}$ & $\begin{array}{l}2.705 \\
2.429\end{array}$ & $\begin{array}{l}0.598 \\
0.540\end{array}$ \\
\hline 5.0 & $\frac{1}{2}$ & $\begin{array}{l}4.917 \\
6.054\end{array}$ & $\begin{array}{l}3.201 \\
4.152\end{array}$ & $\begin{array}{l}1.716 \\
1.902\end{array}$ & $\begin{array}{l}0.556 \\
0.458\end{array}$ \\
\hline 5.5 & $\frac{1}{2}$ & $\begin{array}{l}6.754 \\
5.793\end{array}$ & $\begin{array}{l}4.501 \\
4.130\end{array}$ & $\begin{array}{l}2.243 \\
1.663\end{array}$ & $\begin{array}{l}0.498 \\
0.403\end{array}$ \\
\hline 6.0 & $\frac{2}{2}$ & $\begin{array}{l}7.209 \\
5.085\end{array}$ & $\begin{array}{l}4.908 \\
3.610\end{array}$ & $\begin{array}{l}2.301 \\
1.475\end{array}$ & $\begin{array}{l}0.468 \\
0.008\end{array}$ \\
\hline
\end{tabular}


TABLE XXXIII (Oont.)

MOTSTURE CONTERT DA BA

Run 6

Thene rmy nt. Wot

Hire. Ho. Sermio

Cans.

6.8

$\frac{1}{2}$

4.549

6.111

7.0

$\frac{1}{8}$

6.025

5,448

7.5

1

4.586

4.474

Semp

anc.

8.846

4. 234

$3.414 \quad 1.611$

4.856

1,192

8.889
3.805
0.667

8.0

2

6,986

4.840

4.778
4.236

1.178

0.404

8.5

4.989

4.839

3.792

4,216

1.148

0.685

0.0

4.680

4.288

3.794

0.886

0,358

$3.481 \quad 0.980$

$9.5 \quad \frac{1}{2}$

6,341

4.808

10.0

5.196

4.286

4.668

0.588

8.667

4.084

4.814

4.367

4.796

4.361

0.018

0,006

3.958

0.088

4.041

$\begin{array}{lll}5.438 & 5.279 & 0.159\end{array}$

$12.0 \quad \frac{1}{2}$

$\frac{1}{2}$

Cone Nolnt
per Gatid

0.544

0.472

0.280

0.577

0.140

0.247

0.008

0.303

0.248

0.234

0.088

0.270

0.213

0.100

0.004

0.001

0.021

0.050 


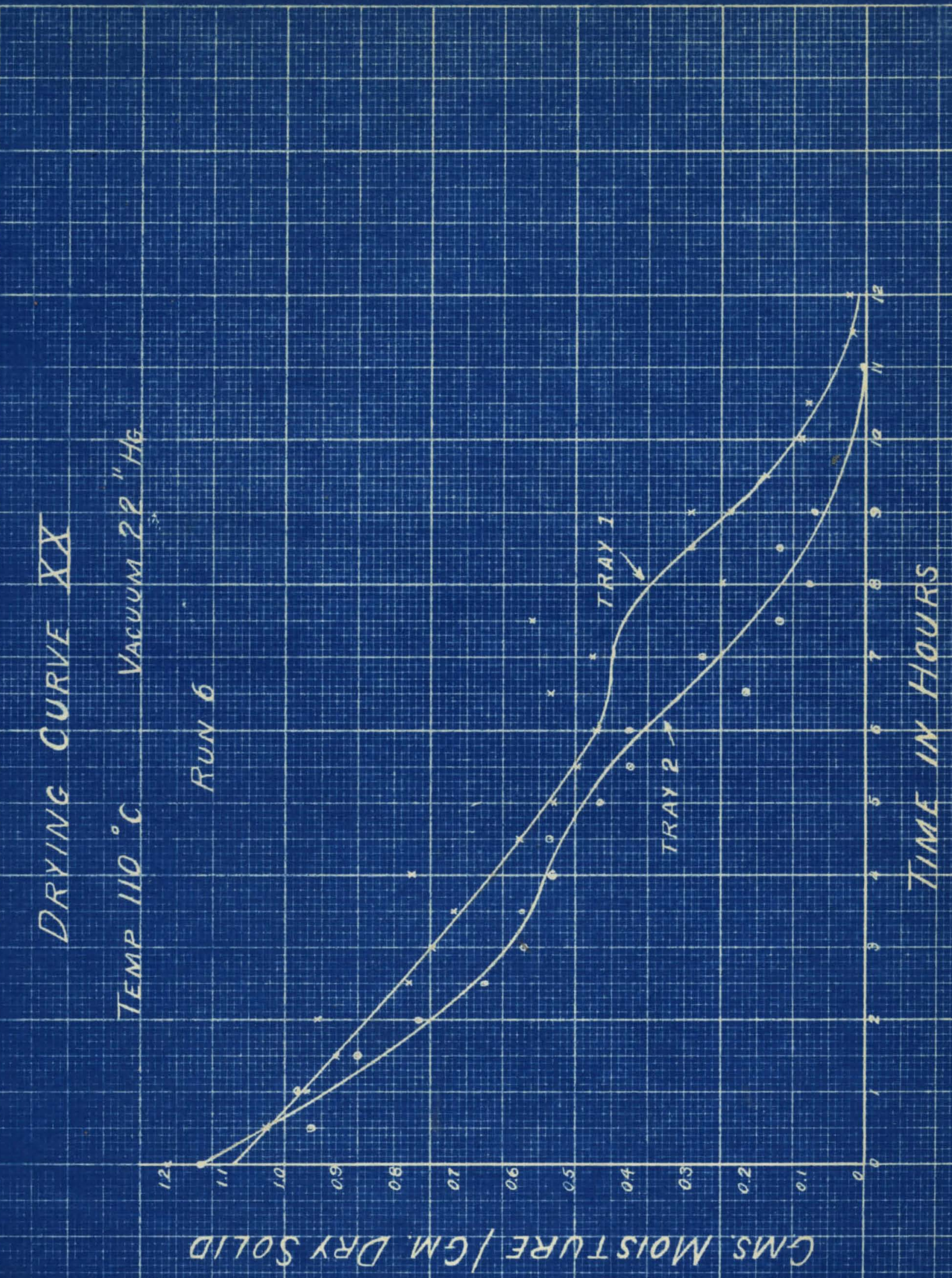


TABLE XXXN

LOQ SEEET

$\operatorname{Tan} 7$

Dopartmont of Chomlcel Englneovins

Spoed solent1f10 Sohool

Univeralty of Loufavilie

DRYINO ON PRUSSIAN BLUS

Date: $5 / 8 / 34$

Temperature: $180^{\circ} \mathrm{C}$.

Va ourum: 28" $\mathrm{Hg}$.

PIent Record Data

Laboratory Data

Hame: Steol Blue

Rx. No.: 4006

Silp No.: 751

Batoh No.: 4

Dete struck: $3 / 29 / 34$

Dete In: $4 / 30 / 34$

No. Tray: Dried: 5

Batoh Rod.: $4 / 30 / 34$
Batch Rtdi $5 / 13 / 34$

Avg. Control Temp: $120.38^{\circ} \mathrm{C}$.

Tray Data




TABLS XXXV

CAX TATRRATURB DAT

Rua 7

Timo

Bre.

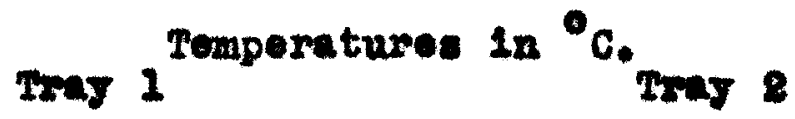

42 \#3 \#4 AVB. \#5 \#6 \#7 ATE.

$\begin{array}{llllllllll}0.85 & 38.5 & 33.5 & 34.0 & 34.3 & 36.0 & 35.5 & 33.6 & 34.0\end{array}$

$0.60 \quad 68.0 * 48.5 \quad 51.0 \quad 49.7 \quad 48.0 \quad 44.0 \quad 47.0 \quad 46.3$

$0.78 \quad 68.0 * 65.5 \quad 62.0 \quad 63.7 \quad 63.0 \quad 64.0 \quad 62.5,65.8$

$1.00 \quad 86.0 \% 67.0 \quad 67.5 \quad 67.8 \quad 67.0 \quad 67.0 \quad 67.0^{\circ} 67.0$

$\begin{array}{llllllll}1.86 & 87.0 * 68.0 & 67.0 & 67.6 & 67.0 & 67.0 & 67.0 & 67.0\end{array}$

$1.00 \quad 86.0 * 67.0 \quad 67.0 \quad 67.0 \quad 65.0 \quad 05.0 \quad 65.0 \quad 65.7$

$1.75 \quad 66.0 \quad 64.8 \quad 64.0 \quad 64.5 \quad 64.5 \quad 64.0 \quad 64.0 \quad 64.8$

$\begin{array}{lllllllll}2.00 & 68.0 & 64.5 & 64.0 & 64.5 & 64.0 & 64.0 & 64.0 & 64.0\end{array}$

$\begin{array}{lllllllll}8.25 & 66.0 & 65.5 & 65.0 & 65.5 & 66.0 & 68.5 & 68.0 & 66.8\end{array}$

$\begin{array}{lllllllll}8.60 & 68.0 & 65.5 & 65.0 & 68.5 & 68.5 & 65.0 & 68.0 & 65.2\end{array}$

$\begin{array}{lllllllll}2.78 & 66.0 & 65.5 & 65.0 & 65.5 & 65.5 & 65.0 & 65.0 & 65.2\end{array}$

$\begin{array}{lllllllll}3.00 & 60.0 & 65.5 & 65.0 & 65.6 & 65.5 & 68.0 & 68.0 & 68.2\end{array}$

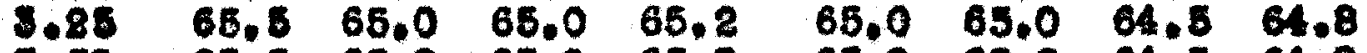

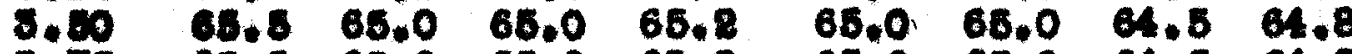

$\begin{array}{lllllllll}3.75 & 68.5 & 65.0 & 65.0 & 65.2 & 65.0 & 65.0 & 64.5 & 64.8\end{array}$

$\begin{array}{lllllllll}4.00 & 65.5 & 65.0 & 66.0 & 65.8 & 65.0 & 65.0 & 64.8 & 64.8\end{array}$

$\begin{array}{lllllllll}4.85 & 65.5 & 65.0 & 65.0 & 65.2 & 65.2 & 65.2 & 64.5 & 64.8\end{array}$

$\begin{array}{lllllllll}4.60 & 66.0 & 65.0 & 66.5 & 65.5 & 65.0 & 68.0 & 68.0 & 68.0\end{array}$

$\begin{array}{lllllllll}4.75 & 66.0 & 65.5 & 65.0 & 65.5 & 66.5 & 65.0 & 65.0 & 65.8\end{array}$

$\begin{array}{lllllllll}8.00 & 66.0 & 68.5 & 66.0 & 65.8 & 66.0 & 65.0 & 65.0 & 66.8\end{array}$

$\begin{array}{lllllllll}5.25 & 66.0 & 65.5 & 66.0 & 65.8 & 66.0 & 65.0 & 65.0 & 66.3\end{array}$

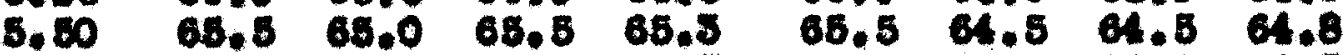

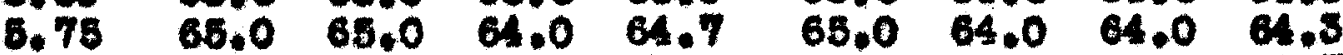

$\begin{array}{lllllllll}6.00 & 65.0 & 65.0 & 63.5 & 64.5 & 64.5 & 65.5 & 63.0 & 63.7\end{array}$

$\begin{array}{lllllllll}6.85 & 65.0 & 65.0 & 65.0 & 65.0 & 64.5 & 64.5 & 64.0 & 64.3\end{array}$

$\begin{array}{lllllllll}6.50 & 65.5 & 64.5 & 65.0 & 65.0 & 65.6 & 64.0 & 64.0 & 64.6\end{array}$

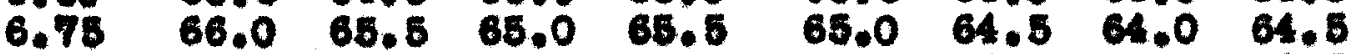

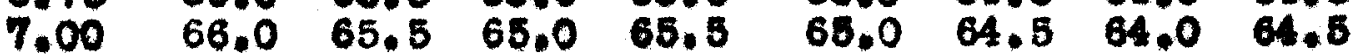

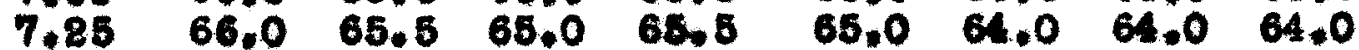

$\begin{array}{lllllllll}7.50 & 66.0 & 66.5 & 65.5 & 65.7 & 65.0 & 65.5 & 64.5 & 64.3\end{array}$

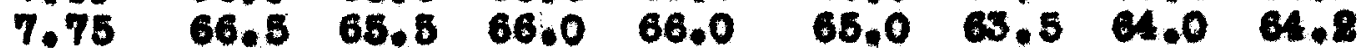

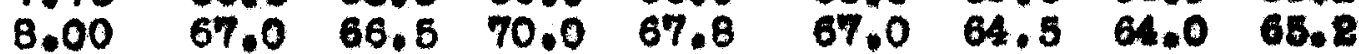

$\begin{array}{lllllllll}8.25 & 70.0 & 66.5 & 70.8 & 71.7 & 69.0 & 64.0 & 65.5 & 66.8\end{array}$

$\begin{array}{lllllllll}8.50 & 74.0 & 80.5 & 94.0 & 88.8 & 7.0 & 65.0 & 78.5 & 68.8\end{array}$

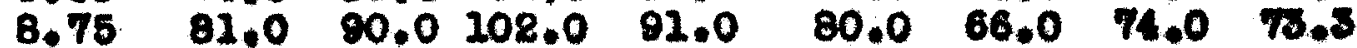

$\begin{array}{llllllll}9.00 \quad 9.0101 .0 & 112.0 & 108.0 & 84.0 & 69.0 & 81.0 & 78.0\end{array}$

$8.25105 .0106 .0115 .0108 .0 \quad 86.0 \quad 76.0 \quad 86.0 \quad 85.0$

$8.50 \quad 115.0114 .0118 .0116 .0 \quad 97.0 \quad 88.0 \quad 95.0 \quad 02.5$

$9.76 \quad 218.6118 .5115 .0 \quad 113.7 \quad 108.5 \quad 05.8 \quad 99.5 \quad 09.8$

10.00119 .8118 .5180 .0119 .5118 .0115 .5128 .0115 .8

* VeIne culted in avermgo. 


\section{CAKE TEMPERATURE CURVE $X X I$}

TEMP $120^{\circ} \mathrm{G}$ VacuUn $22^{\prime H C}$

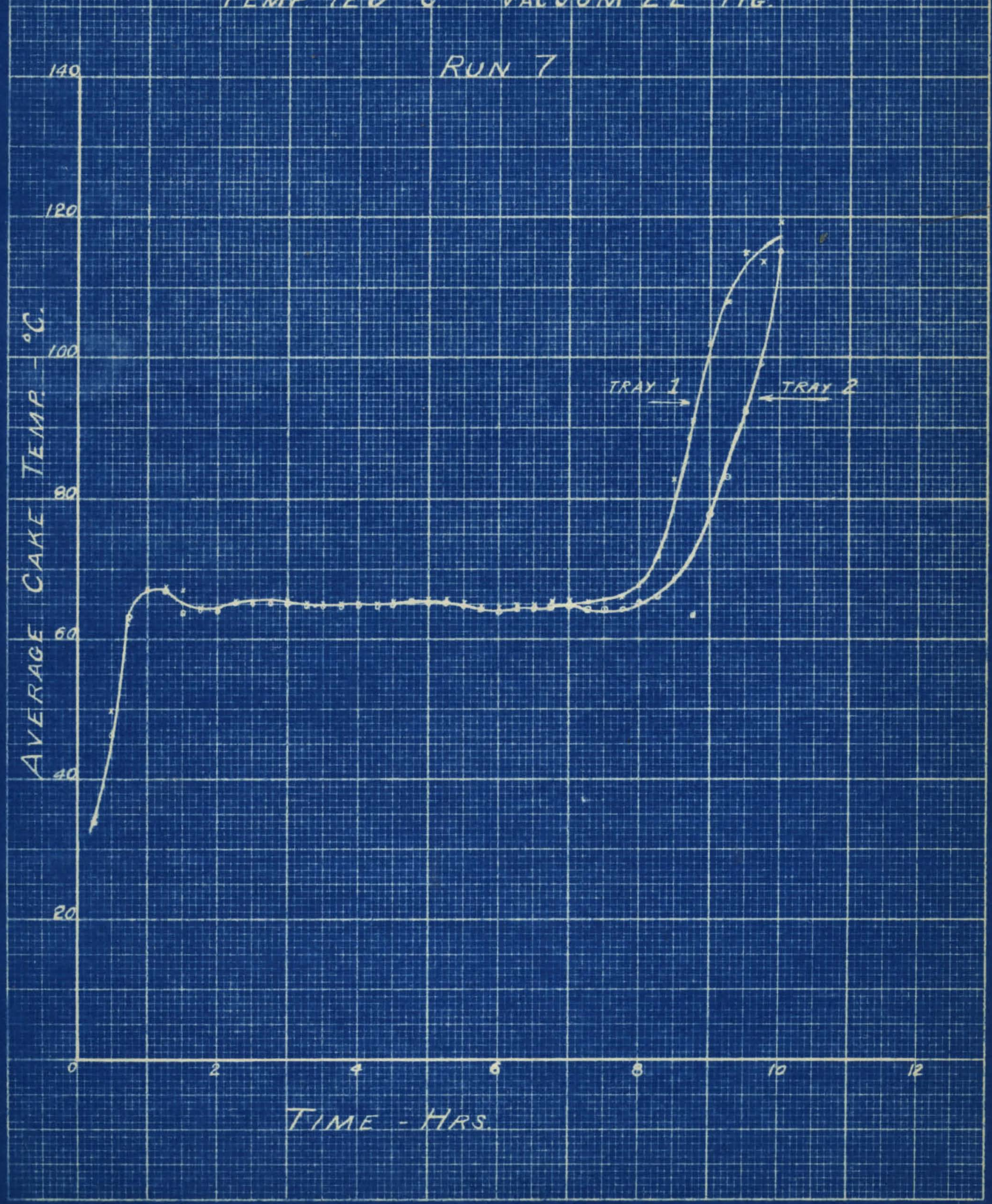


TABLE XXXVI

MOISTURE CONTEET DATA

Run 7

\begin{tabular}{|c|c|c|c|c|c|}
\hline $\begin{array}{l}\text { Time } \\
\text { Hrs. }\end{array}$ & $\begin{array}{c}\text { Tray } \\
\text { No. }\end{array}$ & $\begin{array}{l}\text { Wt. Wot } \\
\text { Samplo } \\
\text { Gms. }\end{array}$ & $\begin{array}{c}\text { Wt. Dry } \\
\text { Sample } \\
\text { Oms. }\end{array}$ & $\begin{array}{l}\text { Molature } \\
\text { Lont } \\
\text { Cmo. }\end{array}$ & $\begin{array}{l}\text { Gns. Molat } \\
\text { per Gm. } \\
\text { Dry Solid }\end{array}$ \\
\hline 0 & $\frac{1}{2}$ & $\begin{array}{l}4.431 \\
4.283\end{array}$ & $\begin{array}{l}2.122 \\
2.100\end{array}$ & $\begin{array}{l}2.309 \\
2.174\end{array}$ & $\begin{array}{l}1.087 \\
1.030^{\circ}\end{array}$ \\
\hline 0.5 & $\begin{array}{l}1 \\
2\end{array}$ & $\begin{array}{l}3.372 \\
3.631\end{array}$ & $\begin{array}{l}1.696 \\
1.818\end{array}$ & $\begin{array}{l}1.676 \\
1.813\end{array}$ & $\begin{array}{l}0.988 \\
0.997\end{array}$ \\
\hline 1.0 & $\begin{array}{l}1 \\
2\end{array}$ & $\begin{array}{l}4.398 \\
5.978\end{array}$ & $\begin{array}{l}2.392 \\
3.311\end{array}$ & $\begin{array}{l}2.001 \\
2.667\end{array}$ & $\begin{array}{l}0.837 \\
0.807\end{array}$ \\
\hline 1.5 & $\frac{1}{2}$ & $\begin{array}{l}6.201 \\
5.912\end{array}$ & $\begin{array}{l}3.255 \\
3.276\end{array}$ & $\begin{array}{l}2.846 \\
2.656\end{array}$ & $\begin{array}{l}0.874 \\
0.805\end{array}$ \\
\hline 8.0 & $\frac{1}{2}$ & $\begin{array}{l}7.848 \\
4.565\end{array}$ & $\begin{array}{l}4.348 \\
2.646\end{array}$ & $\begin{array}{l}3.500 \\
1.910\end{array}$ & $\begin{array}{l}0.804 \\
0.724\end{array}$ \\
\hline 2.5 & $\begin{array}{l}2 \\
2\end{array}$ & $\begin{array}{l}5.918 \\
6.152\end{array}$ & $\begin{array}{l}3.374 \\
3.899\end{array}$ & $\begin{array}{l}2.544 \\
2.853\end{array}$ & $\begin{array}{l}0.755 \\
0.578\end{array}$ \\
\hline 3.0 & $\begin{array}{l}1 \\
2\end{array}$ & $\begin{array}{l}5.573 \\
5.258\end{array}$ & $\begin{array}{l}3.368 \\
3.340\end{array}$ & $\begin{array}{l}2.211 \\
1.918\end{array}$ & $\begin{array}{l}0.658 \\
0.574\end{array}$ \\
\hline 3.5 & $\begin{array}{l}1 \\
2\end{array}$ & $\begin{array}{l}7.732 \\
5.422\end{array}$ & $\begin{array}{l}5.004 \\
2.534\end{array}$ & $\begin{array}{l}2.728 \\
1.888\end{array}$ & $\begin{array}{l}0.546 \\
0.534\end{array}$ \\
\hline 4,0 & $\begin{array}{l}1 \\
2\end{array}$ & $\begin{array}{l}5.894 \\
6.891\end{array}$ & $\begin{array}{l}3.995 \\
4.631\end{array}$ & $\begin{array}{l}1.890 \\
2.260\end{array}$ & $\begin{array}{l}0.475 \\
0.488\end{array}$ \\
\hline 4.5 & $\begin{array}{l}1 \\
2\end{array}$ & $\begin{array}{l}6.123 \\
4.980\end{array}$ & $\begin{array}{l}4.245 \\
3 / 946\end{array}$ & $\begin{array}{l}1.878 \\
1.034\end{array}$ & $\begin{array}{l}0.442 \\
0.262\end{array}$ \\
\hline 5.0 & $\frac{1}{2}$ & $\begin{array}{l}7.367 \\
7.598\end{array}$ & $\begin{array}{l}5.302 \\
5.731\end{array}$ & $\begin{array}{l}2.065 \\
1.867\end{array}$ & $\begin{array}{l}0.380 \\
0.326\end{array}$ \\
\hline 5.6 & $\frac{1}{2}$ & $\begin{array}{l}5.566 \\
6.192\end{array}$ & $\begin{array}{l}3.985 \\
4.577\end{array}$ & $\begin{array}{l}1.581 \\
1.625\end{array}$ & $\begin{array}{l}0.397 \\
0.393\end{array}$ \\
\hline 6.0 & $\frac{1}{2}$ & $\begin{array}{l}4.694 \\
4.932\end{array}$ & $\begin{array}{l}3.520 \\
4.022\end{array}$ & $\begin{array}{l}1.174 \\
0.910\end{array}$ & $\begin{array}{l}0.334 \\
0.226\end{array}$ \\
\hline
\end{tabular}


TABLE XXXNI (Cont.)

MOISTVRE CONTEHT DATA

fiun 7

\begin{tabular}{|c|c|c|c|c|c|}
\hline Tims. & $\begin{array}{l}\text { moy } \\
\text { No. }\end{array}$ & $\begin{array}{l}\text { Wt. Wot } \\
\text { Samplo } \\
\text { Cms. }\end{array}$ & $\begin{array}{c}\text { Wt. Dey } \\
\text { samplo } \\
\text { ome. }\end{array}$ & $\begin{array}{l}\text { Molsture } \\
\text { Lost } \\
\text { Gmil. }\end{array}$ & $\begin{array}{l}\text { Coma. Koint } \\
\text { per Ome } \\
\text { Der solid }\end{array}$ \\
\hline 6.5 & $\frac{1}{2}$ & $\begin{array}{l}4.505 \\
3.022\end{array}$ & $\begin{array}{l}3.807 \\
3.851\end{array}$ & $\begin{array}{l}0.988 \\
1.171\end{array}$ & $\begin{array}{l}0.885 \\
0.804\end{array}$ \\
\hline 7.0 & $\frac{1}{8}$ & $\begin{array}{l}3.762 \\
3.425\end{array}$ & $\begin{array}{l}3.266 \\
2.854\end{array}$ & $\begin{array}{l}0.608 \\
0.860\end{array}$ & $\begin{array}{l}0.190 \\
0.808\end{array}$ \\
\hline 7.5 & $\frac{1}{2}$ & $\begin{array}{l}5.053 \\
4.329\end{array}$ & $\begin{array}{r}4.491 \\
8.651\end{array}$ & $\begin{array}{l}0.848 \\
0.788\end{array}$ & $\begin{array}{l}0.181 \\
0.283\end{array}$ \\
\hline 8.0 & $\frac{1}{2}$ & $\begin{array}{l}4.752 \\
4.574\end{array}$ & $\begin{array}{l}4.452 \\
4.106\end{array}$ & $\begin{array}{l}0.386 \\
0.468\end{array}$ & $\begin{array}{l}0.074 \\
0.214\end{array}$ \\
\hline 8.8 & $\frac{1}{2}$ & $\begin{array}{l}4.055 \\
4.303\end{array}$ & $\begin{array}{l}3.780 \\
3.836\end{array}$ & $\begin{array}{l}0.268 \\
0.467\end{array}$ & $\begin{array}{l}0.069 \\
0.122\end{array}$ \\
\hline 9.0 & $\frac{1}{8}$ & $\begin{array}{l}3.704 \\
4.060\end{array}$ & $\begin{array}{l}3.950 \\
3.026\end{array}$ & $\begin{array}{l}0.174 \\
0.134\end{array}$ & $\begin{array}{l}0.049 \\
0.054\end{array}$ \\
\hline 0.5 & $\frac{1}{2}$ & $\begin{array}{l}6.028 \\
3.820\end{array}$ & $\begin{array}{l}6.028 \\
3.817\end{array}$ & $\begin{array}{l}0.000 \\
0.005\end{array}$ & $\begin{array}{l}0.000 \\
0.001\end{array}$ \\
\hline 0.0 & $\frac{1}{2}$ & 4,142 & 4.137 & 0.008 & 0,001 \\
\hline
\end{tabular}




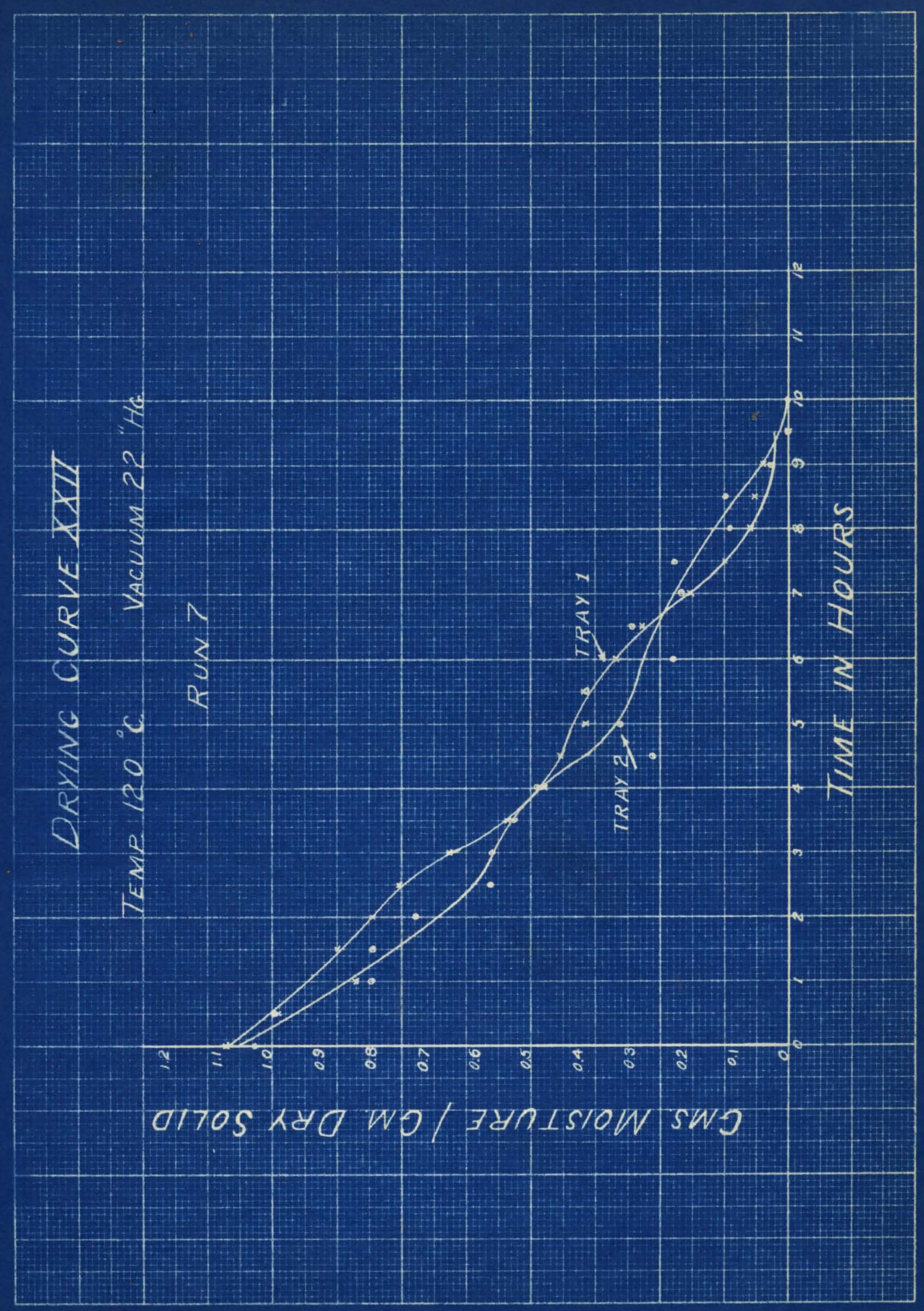




\section{RABLE XXXNII}

\section{LOO SEEET}

Fun 8

Department of Chemlonl Bnglneering

Speed Selentiflo Sohoel Univeralty of Lecileville

\section{DRYIIG OF PRUSSIAR BLUE}

\section{Date: $5 / 9 / 34$}

Temperature: $150^{\circ} \mathrm{C}$.

Vacuum! $22^{\text {" HB. }}$

Plent Rocord Data

Tamo: Stoel BIne

Rx. Ho.: 4006

S11p No.: 731

Batch No.: 4 Date strick: 3/29/34

Ho. Treyt Dried: 5

Date In: $4 / 30 / 34$
Tray Data

\begin{tabular}{|c|c|c|c|c|}
\hline Tray & $\begin{array}{l}\text { Wt. of Cake } \\
\text { Before Dry } 1 \text { tos } \\
\text { ibe. }\end{array}$ & $\begin{array}{l}\text { Tt. of Cake } \\
\text { After Dirglng } \\
\text { Iba. }\end{array}$ & Wt. & $\begin{array}{l}\text { Mols ture } \\
\text { Lost } \\
\text { Ibs. }\end{array}$ \\
\hline 3 & $\begin{array}{r}5.75 \\
6.60 \\
12.26\end{array}$ & $\begin{array}{l}2.75 \\
3.25 \\
6.00\end{array}$ & & $\begin{array}{r}3.00 \\
3.85 \\
6.256\end{array}$ \\
\hline
\end{tabular}


TALS XXVIII

CATS TELPRRATURE DAW

Ama 8

Trim

Ars.

0.85

0.80

0.75

2.00

1.85

1.80

2.76

8.00

8.85

2. 80

8.76

3.00

3.25

3.80

8.76

4.00

4.85

4.60

4.78

6.00

5.25

6.50

5.75

0.00

6.25

6.80

6.76

7.00

7.25

7.80

7.75

8.00

8.28

0.50

8. 75

9.00
Tomperatures in ${ }^{\circ} \mathrm{C}$.

Trat 1 48 \#5 AVE.

49.0

82.0

$60.0 * 60.0$ 63.065 .8 $63.5 \quad 4.0$ $63.0 \quad 63.0$ $65.0 \quad 65.0$ $6.0 \quad 63.0$ $63.0 \quad 65.6$ 64.584 .8 6.5648 64.564 .7 65.065 .8 $65.0 \quad 65.5$ $68.0 \quad 65.8$ 65.068 .8 68.065 .3 68.0 .68 .8 65.065 .3 65.065 .3 65.065 .3 $65.0 \quad 65.3$ 64.565 .3 67.067 .0 69.068 .8 $74.0 \quad 72.5$ 80.0 .81 .0 $\lim _{0} 0$ 9.0 08.0 96.0 107.5107 .6114 .0109 .7 180.0119 .0 128.5 180.8 286.5124 .5186 .0186 .7 132.6131 .0181 .5152 .7 $138.0 \quad 131.0129 .5230 .8$ 234.0234 .0234 .0134 .0 185.0138 .0129 .0131 .0 $134.0 \quad 283.0 \quad 132.5138 .8$ $131.0 \quad 131.0 \quad 130.0130 .7$ $133.0131 .5131,0131.0$

\section{Trat 2} $\$ 6$ \#7 AV.

$\begin{array}{llll}43.0 & 43.0 & 43.6 & 43.2\end{array}$ $62.0 \quad 63.0 \quad 64.0 \quad 63.0$ $63.0 \quad 68.5 \quad 64.5^{\prime} 65.7$ $65.0 \quad 65.0 \quad 68.0 \quad 68.7$ $65.0 \quad 68.0 \quad 62.0 \quad 62.7$ $65.0 \quad 65.0 \quad 69.0 \quad 62.7$ $65.0 \quad 63.8 \quad 64.0 \quad 63.5$ $64.0 \quad 64.8 \quad 64.5 \quad 64.8$ $68.064 .5 \quad 04.5 \quad 64.7$ $64.5 \quad 64.564 .564 .5$ 64.064 .564 .564 .3 64.0 64.5 64.5 64.5 $65.0 \quad 65.0 \quad 64.0 \quad 64.7$ $68.0 \quad 64.5 \quad 64.5 \quad 64.7$ $65.0 \quad 4.5 \quad 64.5 \quad 64.7$ $68.0 \quad 64.5 \quad 64.8 \quad 64.7$ $05.9 \quad 4.5 \quad 64.6 \quad 64.7$ $63.0 \quad 64.8 \quad 64.5 \quad 64.7$ $65.0 \quad 64.5 \quad 64.8 \quad 64.7$ $65.0 \quad 64.5 \quad 64.5 \quad 64.7$ $65.0 \quad 04.0 \quad 64.0 \quad 64.3$ $65.0 \quad 65.8 \quad 64.5 \quad 65.0$ $66.5 \quad 65.8 \quad 65.5 \quad 65.8$ $65.0 \quad 67.0 \quad 66.0 \quad 66.0$ $67.5 \quad 67.5 \quad 66.5 \quad 66.8$ $67.0 \quad 67.0 \quad 66.5 \quad 66.7$ $66.5 \quad 68.5 \quad 66.5 \quad 67.8$ $\begin{array}{llll}66.5 & 70.5 & 66.5 & 67.8\end{array}$ $67.9 \% 5.5 \quad 70.5 \quad 10.5$ $74.0 \quad 86.5 \quad 88.5 \quad 82.0$ $88.0 \quad 99.0 \quad 99.0 \quad 95.3$ 104.0112 .5112 .5100 .7 114.0116 .5117 .0116 .8 182.0123 .0123 .0182 .7 124.0186 .5123 .6184 .5 120.018360127 .6187 .8

* Value oulttod in averago 


\section{CAKE TEMPERATURE CURVE XXIII}

TEMP $130^{\circ} \mathrm{C}$ VACUUM $22^{\prime H} \mathrm{HC}$

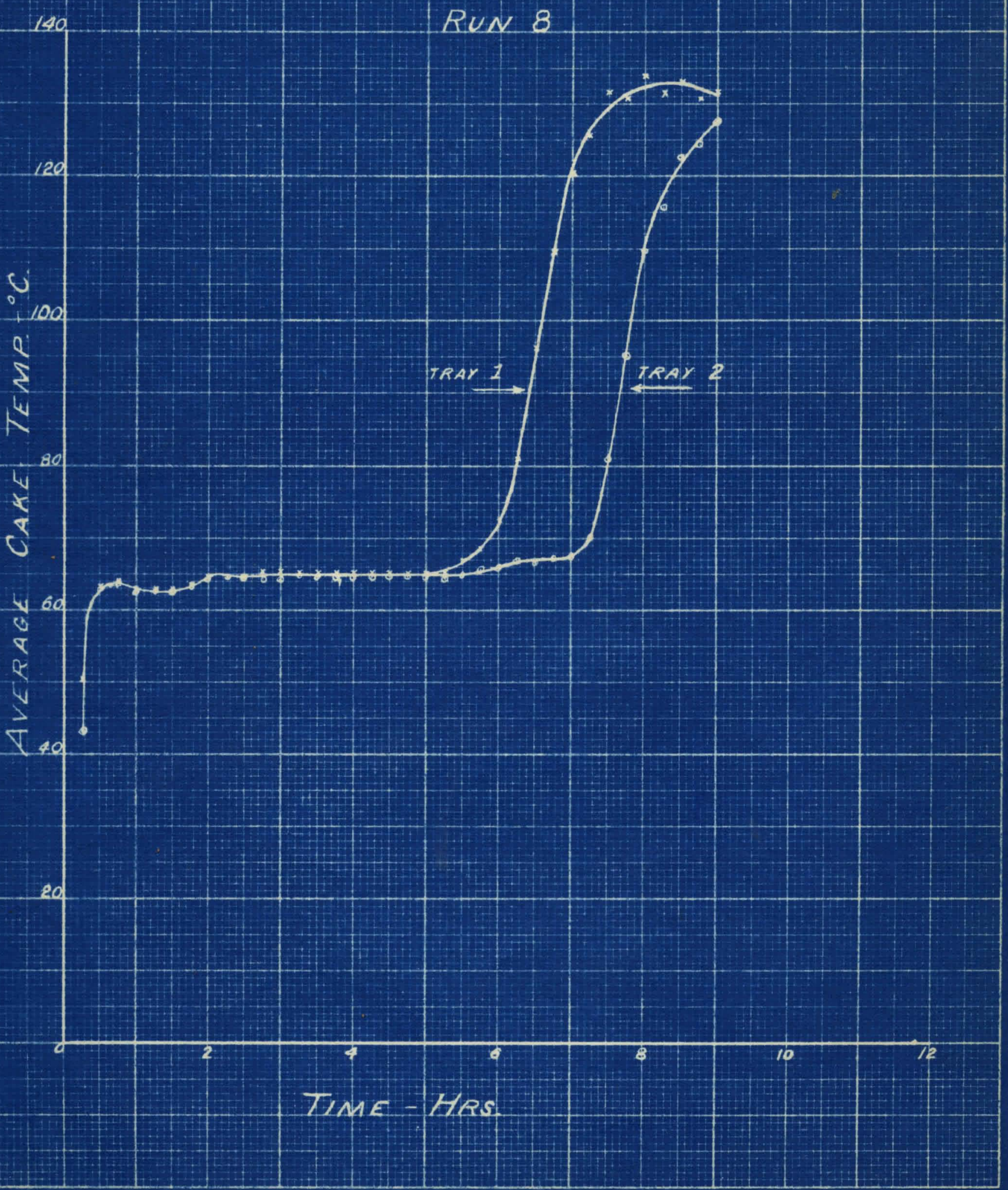


TABLS $\times X \times 1 X$

MOISWURE CORTEET DATA

$\operatorname{Ran} 8$

\begin{tabular}{|c|c|c|c|c|c|}
\hline $\begin{array}{l}\text { Tymo: } \\
\text { Hirg. }\end{array}$ & $\begin{array}{l}\text { Tray } \\
\text { No. }\end{array}$ & $\begin{array}{l}\text { Wt. Wot } \\
\text { Samplo } \\
\text { Gms. }\end{array}$ & $\begin{array}{l}\text { Wt. Dary } \\
\text { Sample } \\
\text { Ome. }\end{array}$ & $\begin{array}{l}\text { Moletare } \\
\text { Lost } \\
\text { Coss. }\end{array}$ & $\begin{array}{l}\text { Mom Molet. } \\
\text { por Ond } \\
\text { Dery solid }\end{array}$ \\
\hline 0 & $\frac{1}{2}$ & $\begin{array}{l}4.028 \\
4.786\end{array}$ & $\begin{array}{l}1.860 \\
8.881\end{array}$ & $\begin{array}{l}8,184 \\
2,268\end{array}$ & $\begin{array}{l}1.160 \\
0.898\end{array}$ \\
\hline 0.8 & $\frac{2}{8}$ & $\begin{array}{l}5,426 \\
8,846\end{array}$ & $\frac{0.087}{8.588}$ & $\begin{array}{l}8.509 \\
2.454\end{array}$ & $\begin{array}{l}0.800 \\
0.728\end{array}$ \\
\hline 1.0 & $\frac{2}{2}$ & $\begin{array}{l}4.512 \\
5.890\end{array}$ & $\begin{array}{l}2.661 \\
3.452\end{array}$ & $\begin{array}{l}1.981 \\
8.558\end{array}$ & $\begin{array}{l}0.768 \\
0.755\end{array}$ \\
\hline 1.5 & $\frac{1}{2}$ & $\begin{array}{l}6.673 \\
4.802\end{array}$ & $\begin{array}{l}3.835 \\
8.912\end{array}$ & $\begin{array}{l}8.858 \\
1.990\end{array}$ & $\begin{array}{l}0.789 \\
0.684\end{array}$ \\
\hline 8,0 & $\frac{1}{2}$ & $\begin{array}{l}6.387 \\
4.076\end{array}$ & $\begin{array}{l}4.085 \\
3.540\end{array}$ & $\begin{array}{l}2.305 \\
1.436\end{array}$ & $\begin{array}{l}0.563 \\
0.106\end{array}$ \\
\hline 2.5 & $\frac{1}{2}$ & $\begin{array}{l}5.028 \\
8.856\end{array}$ & $\begin{array}{l}3.789 \\
4.039\end{array}$ & $\begin{array}{l}2.190 \\
3.790\end{array}$ & $\begin{array}{l}0.589 \\
0.448\end{array}$ \\
\hline 3.0 & $\frac{1}{2}$ & $\begin{array}{l}5.566 \\
5.474\end{array}$ & $\begin{array}{l}3.684 \\
3.906\end{array}$ & $\begin{array}{l}1.888 \\
? .586\end{array}$ & $\begin{array}{l}0.511 \\
0.401\end{array}$ \\
\hline 3.5 & $\frac{1}{2}$ & $\begin{array}{l}6.915 \\
5.659\end{array}$ & $\begin{array}{l}4.771 \\
3.830\end{array}$ & $\begin{array}{l}2.144 \\
1.820\end{array}$ & $\begin{array}{l}0.449 \\
0.478\end{array}$ \\
\hline 4.0 & $\frac{1}{2}$ & $\begin{array}{l}4.171 \\
4.127\end{array}$ & $\begin{array}{l}3.849 \\
3.457\end{array}$ & $\begin{array}{l}0.828 \\
0.690\end{array}$ & $\begin{array}{l}0.884 \\
0.801\end{array}$ \\
\hline 4.5 & $\frac{1}{2}$ & $\begin{array}{l}3.593 \\
4.128\end{array}$ & $\begin{array}{l}3.169 \\
3.532\end{array}$ & $\begin{array}{l}0.484 \\
0.596\end{array}$ & $\begin{array}{l}0.184 \\
0.169\end{array}$ \\
\hline 5.0 & $\begin{array}{l}2 \\
2\end{array}$ & $\begin{array}{l}4.880 \\
5.821\end{array}$ & $\begin{array}{l}4.090 \\
4.665\end{array}$ & $\begin{array}{l}0.730 \\
1.158\end{array}$ & $\begin{array}{l}0.278 \\
0.248\end{array}$ \\
\hline 5.5 & $\frac{1}{2}$ & $\begin{array}{l}4.385 \\
3.767\end{array}$ & $\begin{array}{l}3.863 \\
3.053\end{array}$ & $\begin{array}{l}0.522 \\
0.714\end{array}$ & $\begin{array}{l}0.285 \\
0.254\end{array}$ \\
\hline 6.0 & $\begin{array}{l}1 \\
2\end{array}$ & $\begin{array}{l}3.521 \\
4.340\end{array}$ & $\begin{array}{l}3.217 \\
3.617\end{array}$ & $\begin{array}{l}0.304 \\
0.723\end{array}$ & $\begin{array}{l}0.095 \\
0.200\end{array}$ \\
\hline
\end{tabular}


TABIS XxXIX (Oont.)

MOISTORE COXTENT DATA

Run 8

\begin{tabular}{|c|c|c|c|c|c|}
\hline Thes & $\begin{array}{l}\text { Tray } \\
\text { Mo. }\end{array}$ & $\begin{array}{l}\text { wt. Wot } \\
\text { sinple } \\
\text { ans. }\end{array}$ & $\begin{array}{l}\text { Wt. Day } \\
\text { sampie } \\
\text { Caid. }\end{array}$ & $\begin{array}{l}\text { Moletine } \\
\text { Lost } \\
\text { Oms. }\end{array}$ & $\begin{array}{l}\text { Cuns. Molot } \\
\text { per Cant } \\
\text { Ders solid }\end{array}$ \\
\hline 6.5 & $\frac{2}{8}$ & $\begin{array}{l}8.089 \\
8.080\end{array}$ & $\begin{array}{l}3.881 \\
4.258\end{array}$ & $\begin{array}{l}0.168 \\
0.837\end{array}$ & $\begin{array}{l}0.044 \\
0.197\end{array}$ \\
\hline 7.0 & $\frac{1}{8}$ & $\begin{array}{l}2.458 \\
4.642\end{array}$ & $\begin{array}{l}8.447 \\
4.077\end{array}$ & $\begin{array}{l}0.008 \\
0.864\end{array}$ & $\begin{array}{l}0.008 \\
0.238\end{array}$ \\
\hline 7.6 & $\frac{1}{8}$ & $\begin{array}{l}5.386 \\
5.146\end{array}$ & $\begin{array}{l}5.309 \\
4.641\end{array}$ & $\begin{array}{l}0.017 \\
0.804\end{array}$ & $\begin{array}{l}0.003 \\
0.208\end{array}$ \\
\hline 6.0 & $\begin{array}{l}1 \\
2\end{array}$ & 4.696 & 4.306 & 0.380 & 0.092 \\
\hline 8.6 & $\begin{array}{l}1 \\
2\end{array}$ & 3.686 & 3.670 & 0.007 & 0.008 \\
\hline
\end{tabular}




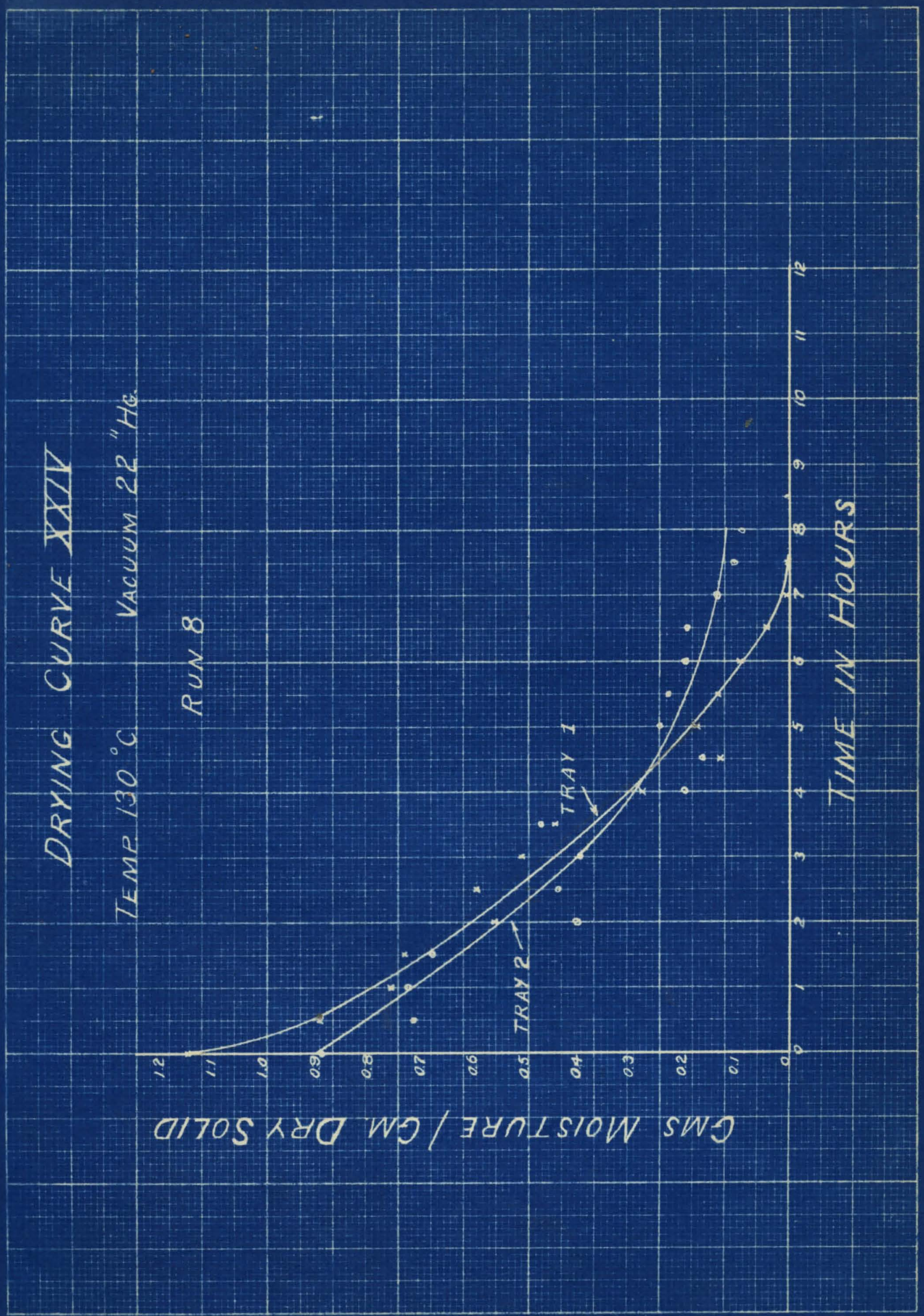


TABLE XI

LOA SHEET

Run 9

Departmont of Chomlosl Bnglnooring

Speod Solont1f10 School

Oniveralty of Loulevilie

\section{DRIIMO OF PROSSIAX BLDE}

Date: $4 / 27 / 34$

Tompereture: $100^{\circ} \mathrm{C}$.

Vecuum: 26" He.

PIant Record Data

Iaboratory Data

Hamo: Steel Blue

Rx. Ho.t 4006

S12p No.: 731

Batoh No.: 4

Date Struok: $3 / 29 / 34$

Do. Traya Dried: 1

Date In: $4 / 27 / 34$

Batoh Rod.: 4/27/34

Batoh Rtd.: $4 / 30 / 34$

Avg. Control Temp: $99.22^{\circ} \mathrm{C}$.

Trey Data

\begin{tabular}{|c|c|c|c|c|}
\hline $\begin{array}{c}\text { Tray } \\
\text { No. }\end{array}$ & $\begin{array}{l}\text { wt. of cake } \\
\text { Before Drying } \\
\text { Ibs. }\end{array}$ & $\begin{array}{l}\text { Wt. of Cake } \\
\text { After prying } \\
\text { ibs. }\end{array}$ & $w t$ & $\begin{array}{l}\text { Molsture } \\
\text { Lost } \\
\text { Ibs. }\end{array}$ \\
\hline 1 & $\begin{array}{r}6.50 \\
6.78 \\
13.25\end{array}$ & $\begin{array}{l}3.25 \\
3.25 \\
6.50\end{array}$ & & $\begin{array}{l}3.25 \\
3.50 \\
6.16\end{array}$ \\
\hline
\end{tabular}


TABLE XLI

CAKS TEUPERATURE DATL

Run 8

T1mo

Hire.

0.85

0.80

0.75

1.00

1.25

1.50

1.75

2.00

2.25

2.50

2.75

3.00

3.25

3.50

3.25

4.00

4.85

4.60

4.75

5,00

5.25

5.50

5.75

6.00

6.25

6.80

6.75

7.00

7.88

7.50

7.75

8.00

8.26

8.60

8.75 0.00
Temperatures in ${ }^{\circ} \mathrm{C}$. \#2 \#3 \#4 AV8. \#6 \#6 \#7 Av8.

80.0

85.0

35.0

44.0

60.0

50.0

50.0

50.0

50.0

50.0

50.0

80.0

50.0

49.5

50.0

50.0

50.0

50.0

50.0

50.0

50.0

50.0

50.0

50.0

50.0

51.0

52.0

54.5

54.0

52.0

54.5

53.0

52.0

52.0

53.0

38.0

$\begin{array}{lll}80.0 & 19.5 & 29.8 \\ 82.5 & 82.5 & 23.8 \\ 34.5 & 32.5 & 33.7 \\ 43.5 & 43.0 & 43.5 \\ 49.5 & 49.0 & 49.5 \\ 49.5 & 49.0 & 49.5 \\ 49.5 & 49.0 & 49.5 \\ 49.5 & 49.0 & 49.5 \\ 49.5 & 49.0 & 49.5 \\ 49.5 & 49.0 & 49.5 \\ 49.5 & 49.0 & 49.5 \\ 49.5 & 49.0 & 49.5 \\ 49.0 & 48.5 & 49.2 \\ 48.5 & 48.0 & 48.5 \\ 49.0 & 49.0 & 49.5 \\ 49.5 & 49.0 & 49.5 \\ 49.5 & 49.0 & 49.5 \\ 49.5 & 49.0 & 49.5 \\ 49.5 & 49.0 & 49.5 \\ 49.5 & 49.0 & 49.5 \\ 49.5 & 49.0 & 49.5 \\ 49.5 & 49.0 & 49.5 \\ 49.5 & 49.0 & 49.5 \\ 49.5 & 49.0 & 49.5 \\ 48.0 & 48.7 & 48.7 \\ 50.0 & 49.5 & 50.8 \\ 50.0 & 49.5 & 50.1 \\ 56.5 & 55.0 & 85.5 \\ 53.5 & 52.0 & 53.2 \\ 51.5 & 51.0 & 51.5 \\ 53.0 & 53.0 & 53.5 \\ 52.5 & 52.0 & 58.5 \\ 51.5 & 52.0 & 51.8 \\ 51.5 & 52.0 & 51.8 \\ 58.5 & 52.0 & 58.5 \\ 54.5 & 52.0 & 52.8 \\ & & \end{array}$

29.022 .5

29.0 21.5 $82.6,20.5$

$25.028 .0 \quad 22.8^{\prime} 23.8$

$37.0 \quad 34.0 \quad 32.5 \quad 34.5$

$47.0 \quad 46.0 \quad 45.0 \quad 46.7$

$\begin{array}{lllll}49.0 & 48.5 & 47.5 & 48.3\end{array}$

$49.5 \quad 48.5$

$49.0 \quad 48.5$

$49.0 \quad 48.5$

$49.0 \quad 49.0$

$49.0 \quad 48.5$

$49.0 \quad 18.5$

$40.0 \quad 48.5$

$48.0 \quad 49.0$

$49.5 \quad 18.5$

$49.0 \quad 48.5$

$49.0 \quad 48.5$

$49.0 \quad 48.5$

$49.0 \quad 48.5$

$49.0 \quad 48.5$

$49.0 \quad 48.5$

$49.0 \quad 48.5$

49.048 .5

$49.0 \quad 48.5$

$49.0 \quad 48.5$

$48.0 \quad 47.5$

48.549 .5

49.5

55.5

52.5

51.0

53.0

52.0

51.0

58.0

52.0

52.0
40.0

$48.8 \quad 48.7$

$48.5 \quad 48.7$

$48.5 \quad 48.7$

$48.5 \quad 48.7$

$48.5 \quad 48.7$

48.548 .7

47.548 .5

48.548 .8

$48.5 \quad 48.7$

$48.5 \quad 48.7$

$48.5 \quad 48.7$

$48.5 \quad 48.7$

48.548 .7

$48.5 \quad 48.7$

48.548 .7

$48.5 \quad 48.7$

$48.5 \quad 48.7$

$48.5 \quad 48.7$

$47.5 \quad 47.7$

$49.0 \quad 49.0$

$49.5 \quad 49.8$

$52.0 \quad 52.3$

$52.0 \quad 51.3$

$51.5 \quad 52.3$

$51.5 \quad 51.3$

51.551 .7

58.558 .3

52.552 .0
$54.5 \quad 55.2$

$51.5 \quad 51.8$ 
TABLE XII (Cont.)

CAKE TREPERATURE DATA

Fun 9

Timo

Hirs.

Tray 1 Tamparatures in ${ }^{\circ} \mathrm{C}$. $\# 2$ \#3 \#4 \#6. \#5 \#6 \#7 Avg.

0.85 $82.0 \quad 56.5 \quad 58$ 52.053 .5 $58.0 \quad 60.0 \quad 55.5 \quad 55.7$ $52.0 \quad 88.5$ 9.50 52.0 0.75 82.0 69.0 38.054 .3 54.0 57.0 61.0 $65.0 \quad 55.0$ 56.0 62.0 54.0 68.7 $58.0 \quad 56.0 \quad 56.7$ 65.061 .0 $53.0 \quad 62.0$ $88.0 \quad 68.0$ $63.0 \quad 57.0$ $67.5 \quad 61.0$ $73.0 \quad 68.0$ 52.0 61.5 61.0 58.7 54.5 61.6 88.0 67.8 55.061 .6 $60.0 \quad 58.7$

79.078 .5 80.578 .5 86.582 .0 $\begin{array}{llllll}58.0 & 65.0 & 61.5 & 61.2 & 89.0 & 86.8\end{array}$ $60.0 \quad 67.0 \quad 64.5$ 63.8 92.0 89.5 $\begin{array}{ll}58.0 & 58.8 \\ 56.0 & 56.8 \\ 56.0 & 58.0 \\ 68.0 & 58.7 \\ 58.5 & 59.6 \\ 60.5 & 65.0 \\ 68.5 & 68.8 \\ 70.5 & 74.0 \\ 74.5 & 77.8 \\ 78.5 & 82.8 \\ 84.8 & 86.7 \\ 88.5 & 80.0\end{array}$ 
CAKE TEMPERATURE CURVE XXV

TEMP $100^{\circ} \mathrm{C}$ VACUUM $26^{\text {"Ha }}$

140 RuN 9

$-120$

S Sien

1
0
4
4

4
5
5
60
4
5
$\frac{5}{4}$
$\frac{1}{x}$
50

$r$



TIMA-HRS. 
TABLE XLIT

MOISTURE COMTBNT DATA

Pun 9

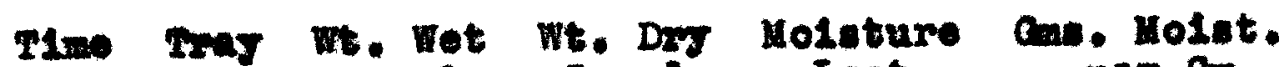

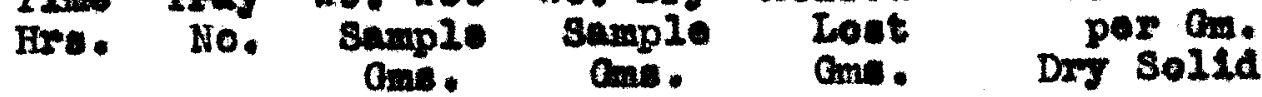

\begin{tabular}{|c|c|c|c|c|c|}
\hline 0 & $\frac{1}{8}$ & $\begin{array}{l}8.435 \\
4.861\end{array}$ & $\begin{array}{l}2.810 \\
2.888\end{array}$ & $\begin{array}{l}2.685 \\
2.273\end{array}$ & $\begin{array}{l}0.988 \\
0.905\end{array}$ \\
\hline 0.5 & $\frac{1}{2}$ & $\begin{array}{l}5.943 \\
4.966\end{array}$ & $\begin{array}{l}8.781 \\
8.656\end{array}$ & $\begin{array}{l}2.468 \\
2.334\end{array}$ & $\begin{array}{l}0.886 \\
0.885\end{array}$ \\
\hline 1.0 & $\frac{1}{2}$ & $\begin{array}{l}5.950 \\
5.374\end{array}$ & $\begin{array}{l}3.175 \\
8.934\end{array}$ & $\begin{array}{l}2.775 \\
2.440\end{array}$ & $\begin{array}{l}0.874 \\
0.838\end{array}$ \\
\hline 1.5 & $\frac{1}{2}$ & $\begin{array}{l}5.250 \\
5.921\end{array}$ & $\begin{array}{l}3.045 \\
3.323\end{array}$ & $\begin{array}{l}2.185 \\
2.588\end{array}$ & $\begin{array}{l}0.7188 \\
0.780\end{array}$ \\
\hline 8.0 & $\begin{array}{l}1 \\
2\end{array}$ & $\begin{array}{l}\mathbf{5 . 8 3 8} \\
\mathbf{5 . 3 4 4}\end{array}$ & $\begin{array}{l}3.169 \\
3.399\end{array}$ & $\begin{array}{l}2.669 \\
2.145\end{array}$ & $\begin{array}{l}0.848 \\
0.638\end{array}$ \\
\hline 2.5 & $\frac{1}{2}$ & $\begin{array}{l}3.817 \\
7.066\end{array}$ & $\begin{array}{l}2.218 \\
4.299\end{array}$ & $\begin{array}{l}1.605 \\
2.767\end{array}$ & $\begin{array}{l}0.727 \\
0.644\end{array}$ \\
\hline 3.0 & $\begin{array}{l}1 \\
2\end{array}$ & $\begin{array}{l}6.277 \\
7.664\end{array}$ & $\begin{array}{l}3.854 \\
4.578\end{array}$ & $\begin{array}{l}2.483 \\
3.086\end{array}$ & $\begin{array}{l}0.630 \\
0.674\end{array}$ \\
\hline$\$ .5$ & $\frac{1}{2}$ & $\begin{array}{l}6.081 \\
4.551\end{array}$ & $\begin{array}{l}4.454 \\
2.810\end{array}$ & $\begin{array}{l}2.587 \\
1.721\end{array}$ & $\begin{array}{l}0.568 \\
0.612\end{array}$ \\
\hline 4.0 & $\begin{array}{l}1 \\
2\end{array}$ & $\begin{array}{l}6.164 \\
5.884\end{array}$ & $\begin{array}{l}4.047 \\
3.798\end{array}$ & $\begin{array}{l}2.127 \\
2.086\end{array}$ & $\begin{array}{l}0.523 \\
0.580\end{array}$ \\
\hline 4.5 & $\frac{1}{2}$ & $\begin{array}{l}5.720 \\
7.478\end{array}$ & $\begin{array}{l}3.678 \\
4.807\end{array}$ & $\begin{array}{l}2.042 \\
2.565\end{array}$ & $\begin{array}{l}0.565 \\
0.528\end{array}$ \\
\hline 5.0 & $\frac{1}{2}$ & $\begin{array}{l}5.494 \\
8.537\end{array}$ & $\begin{array}{l}3.556 \\
5.878\end{array}$ & $\begin{array}{l}1.988 \\
2.668\end{array}$ & $\begin{array}{l}0.465 \\
0.455\end{array}$ \\
\hline B. 5 & $\frac{1}{2}$ & $\begin{array}{l}7.486 \\
6.400\end{array}$ & $\begin{array}{l}5.621 \\
4.531\end{array}$ & $\begin{array}{l}1.866 \\
1.949\end{array}$ & $\begin{array}{l}0.3 \\
0.3\end{array}$ \\
\hline 7.0 & $\frac{1}{2}$ & $\begin{array}{l}6.055 \\
6.385\end{array}$ & $\begin{array}{l}4.351 \\
5.078\end{array}$ & $\begin{array}{l}1.708 \\
1.305\end{array}$ & $\begin{array}{l}0.3 \\
0.2\end{array}$ \\
\hline
\end{tabular}


TABIS XIII (Cont.)

MOISTURE CONTEST DATA

Run 9

\begin{tabular}{|c|c|c|c|c|c|}
\hline Timo & $\begin{array}{l}\text { Tray } \\
\text { No. }\end{array}$ & $\begin{array}{c}\text { Wt. Wot } \\
\text { 8ampio } \\
\text { Onis. }\end{array}$ & $\begin{array}{c}\text { Wt. Dar } \\
\text { sample } \\
\text { Conse. }\end{array}$ & $\begin{array}{l}\text { Moloture } \\
\text { Lost } \\
\text { gane. }\end{array}$ & $\begin{array}{l}\text { Cane. Holet. } \\
\text { per Cant } \\
\text { Dey solid }\end{array}$ \\
\hline 8.8 & $\frac{1}{2}$ & $\begin{array}{l}5.865 \\
5.308\end{array}$ & $\begin{array}{l}4.705 \\
4.762\end{array}$ & $\begin{array}{l}2.160 \\
4.634\end{array}$ & $\begin{array}{l}0.247 \\
0.153\end{array}$ \\
\hline 10.0 & 2 & $\begin{array}{l}6.087 \\
6.003\end{array}$ & $\begin{array}{l}8.887 \\
8.665\end{array}$ & $\begin{array}{l}0.770 \\
0.388\end{array}$ & $\begin{array}{l}0.146 \\
0.060\end{array}$ \\
\hline 21.8 & $\frac{1}{2}$ & $\begin{array}{l}6.001 \\
5.086\end{array}$ & $\begin{array}{l}8.702 \\
4.808\end{array}$ & $\begin{array}{l}0.303 \\
0.121\end{array}$ & $\begin{array}{l}0.058 \\
0.088\end{array}$ \\
\hline 18. & $\frac{1}{8}$ & $\begin{array}{l}5.109 \\
4.800\end{array}$ & $\begin{array}{l}4.758 \\
4.481\end{array}$ & $\begin{array}{l}0.354 \\
0.086\end{array}$ & $\begin{array}{l}0,074 \\
0.006\end{array}$ \\
\hline
\end{tabular}




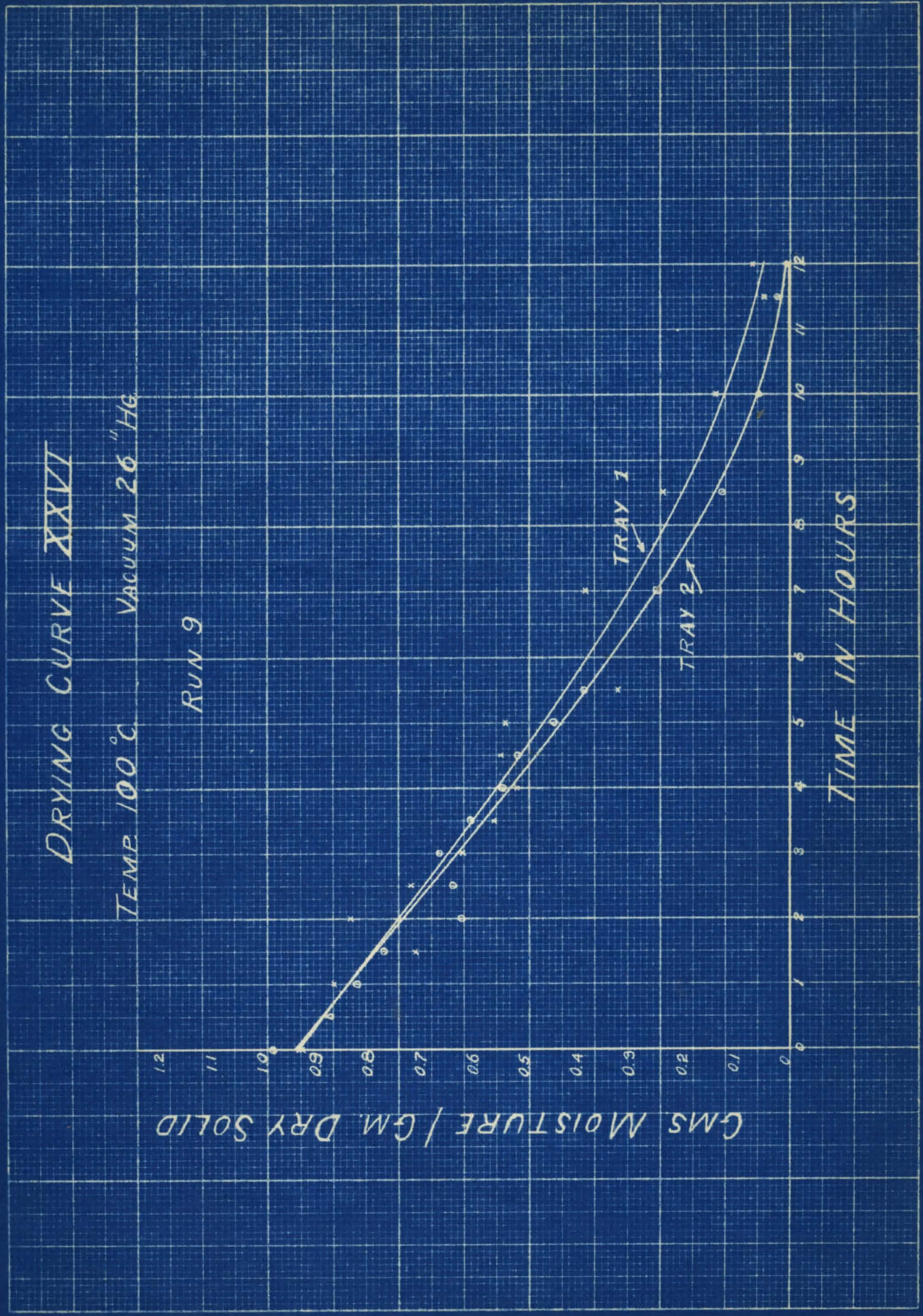




\section{TABLE XLIII}

LOO SHEET

Run 10

Department of Chemical Englneerling

Spoed scientiflo School

University of Lonisvilie

\section{DFYINO OF PRUSSIAN BLUE}

\section{Date: $5 / 2 / 34$}

Temperature: $120^{\circ} \mathrm{C}$.

Vacuum: $26^{\text {" }}$ He.

Plant Record Data

Name: Steel Blue

Rx. No.: 4006

SIIp NO.: 731

Batch No.: 4

Date Struck: $3 / 29 / 34$

No. Trays Dried: 5

Dete In: $4 / 30 / 34$
Batch Rod.: $4 / 30 / 34$

Betch Rtd.: $5 / 12 / 34$

Avg. Control Temp.: $109.10^{\circ} \mathrm{C}$.

Tray Data

\begin{tabular}{|c|c|c|c|}
\hline $\begin{array}{l}\text { Tray } \\
\text { No. }\end{array}$ & $\begin{array}{l}\text { Wt. of Cake } \\
\text { Beofre Dry ing } \\
\text { Ibe. }\end{array}$ & $\begin{array}{l}\text { Wt. of Cake } \\
\text { After Drying } \\
\text { ibs. }\end{array}$ & $\begin{array}{l}\mathrm{W}_{t} \text {. of Molstrare } \\
\text { lost } \\
\text { lbs. }\end{array}$ \\
\hline 1 & $\begin{array}{r}5.50 \\
7.00 \\
12.50\end{array}$ & $\begin{array}{r}2.25 \\
3.25 \\
5.50\end{array}$ & $\begin{array}{l}3.25 \\
3.75 \\
7.00\end{array}$ \\
\hline
\end{tabular}


Run 10

The

Fire

0.28

0.80

0.75

1.00

1.25

1.50

1.75

8.00

8,25

2.80

2.75

3.00

3.85

3.80

5.78

4.00

4.85

4.80

4.75

5.00

5.25

5.50

5.75

6.00

6.25

6.60

6.75

7.00

7.25

7.60

7.75

8.00

8.25

8.50

8.75

9.00

9.25

0.50

9.75

10.00

\section{TABLB XIN} \\ CAKa TEXPERATURE BAT
}

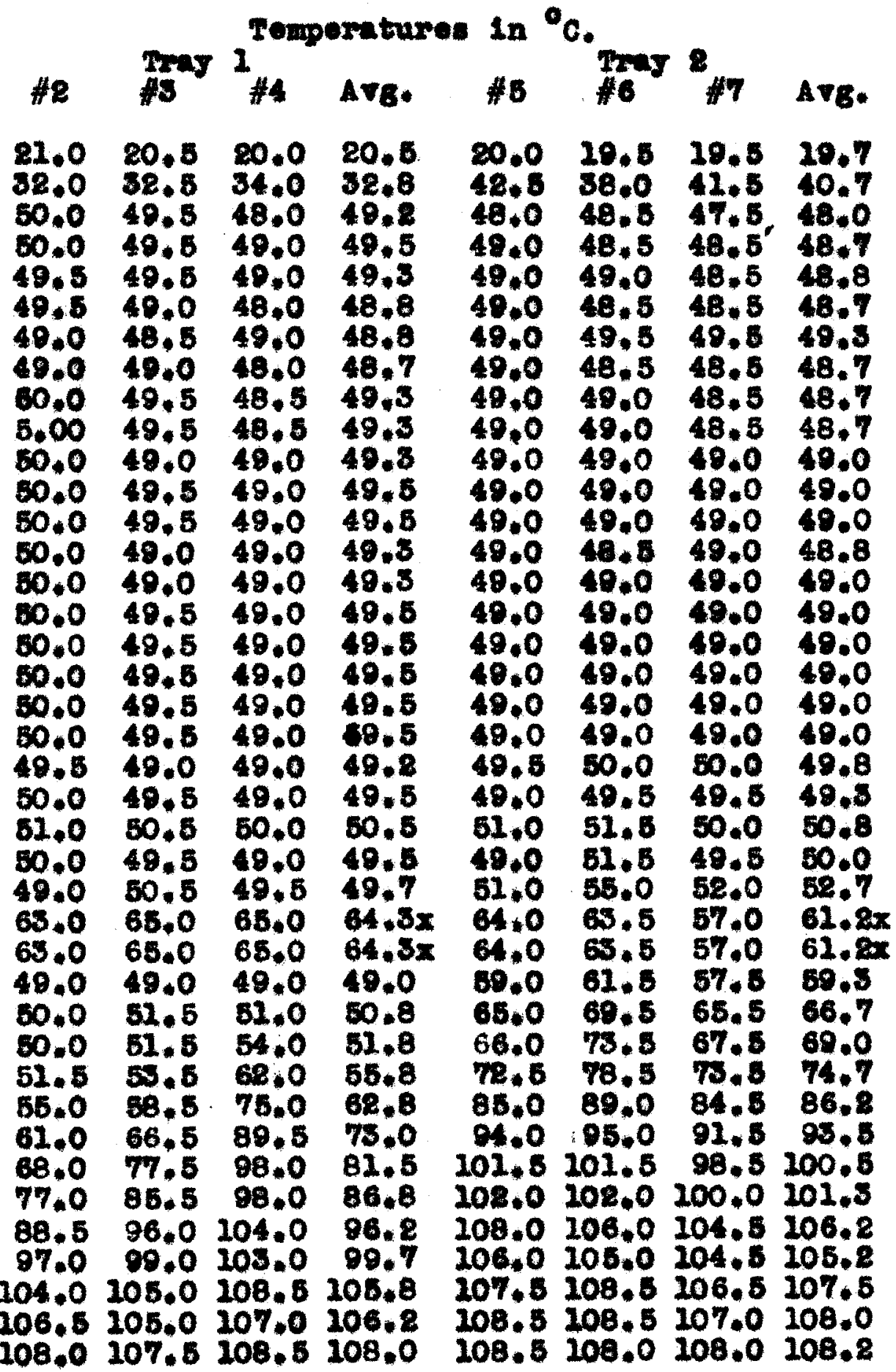

$x$ Value omitted in plotting oake temperature ourve. 
155

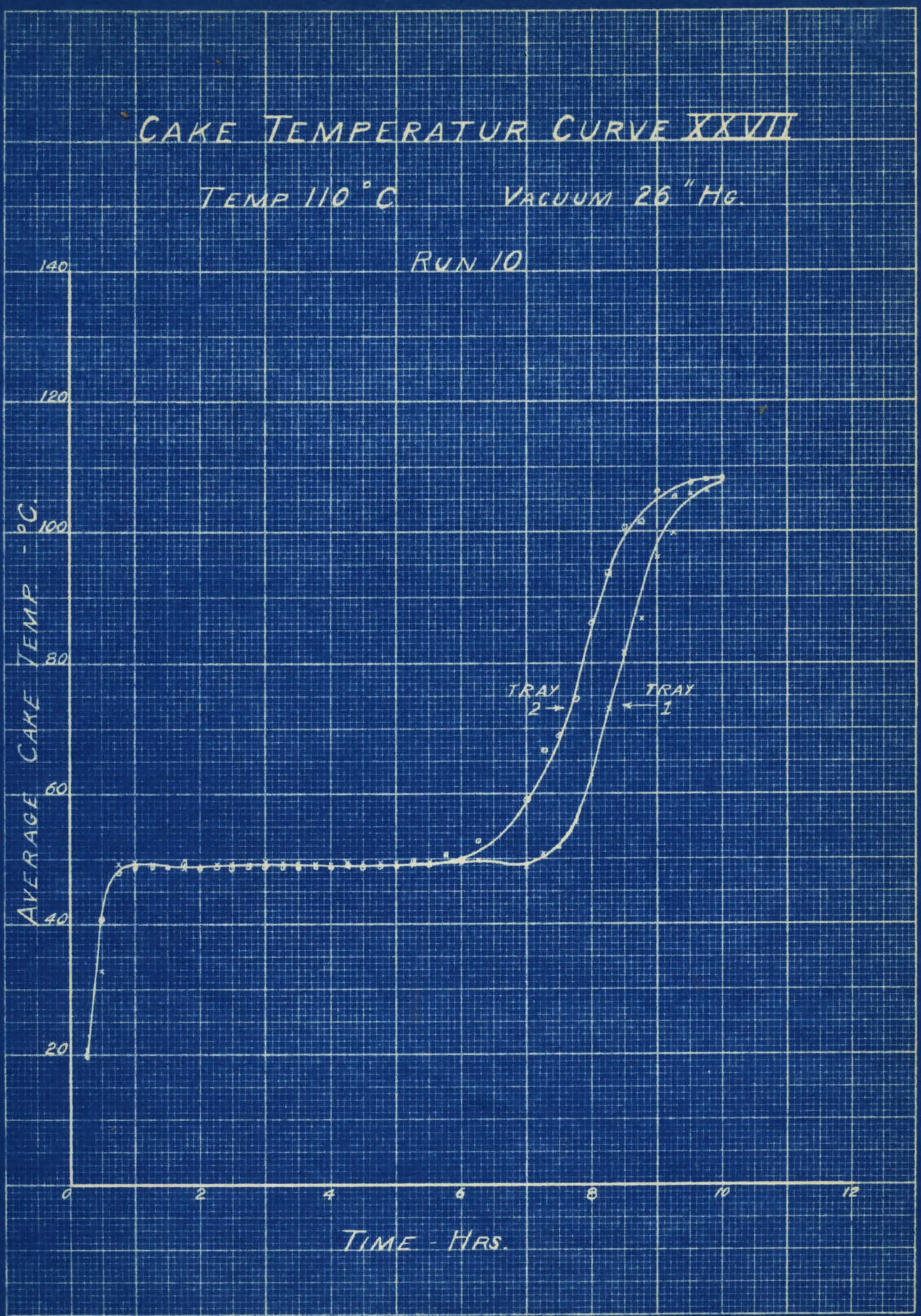


TABLE XDY

MOISTURE CONTENT DATA

Run 10

\begin{tabular}{|c|c|c|c|c|c|}
\hline rimo & $\begin{array}{c}\text { Tray } \\
\text { No. }\end{array}$ & $\begin{array}{c}\text { Wt. Wot } \\
\text { sample } \\
\text { and. }\end{array}$ & $\begin{array}{c}\text { Wt. Dey } \\
\text { Samplo } \\
\text { Case. }\end{array}$ & $\begin{array}{l}\text { Molature } \\
\text { Lost } \\
\text { Cons. }\end{array}$ & $\begin{array}{l}\text { Cun Molat } \\
\text { per and } \\
\text { Dery solio }\end{array}$ \\
\hline 0 & $\frac{1}{2}$ & $\begin{array}{l}4.704 \\
5.082\end{array}$ & $\begin{array}{l}2.875 \\
8.685\end{array}$ & $\begin{array}{l}2.431 \\
8.400\end{array}$ & $\begin{array}{l}7.067 \\
6.888\end{array}$ \\
\hline 0.3 & $\frac{1}{8}$ & $\begin{array}{l}3.905 \\
3.318\end{array}$ & $\begin{array}{l}8.076 \\
2.761\end{array}$ & $\begin{array}{l}1.887 \\
1.852\end{array}$ & $\begin{array}{l}0.881 \\
0.882\end{array}$ \\
\hline 1.0 & $\frac{1}{2}$ & $\begin{array}{l}5.280 \\
6.660\end{array}$ & $\begin{array}{l}2.625 \\
3.657\end{array}$ & $\begin{array}{l}8.456 \\
3.058\end{array}$ & $\begin{array}{l}0.868 \\
0.833\end{array}$ \\
\hline 1.6 & $\frac{1}{8}$ & $\begin{array}{l}6.085 \\
3.897\end{array}$ & $\begin{array}{l}3.568 \\
2.528\end{array}$ & $\begin{array}{l}2.5283 \\
1.568\end{array}$ & $\begin{array}{l}0.709 \\
0.673\end{array}$ \\
\hline 2.0 & $\frac{1}{8}$ & $\begin{array}{l}5.895 \\
7.079\end{array}$ & $\begin{array}{l}3.584 \\
4.423\end{array}$ & $\begin{array}{l}2.311 \\
8.666\end{array}$ & $\begin{array}{l}0.644 \\
0.604\end{array}$ \\
\hline 8.5 & $\begin{array}{l}1 \\
8\end{array}$ & $\begin{array}{l}5.825 \\
6.356\end{array}$ & $\begin{array}{l}3.494 \\
4.448\end{array}$ & $\begin{array}{l}2.338 \\
2.913\end{array}$ & $\begin{array}{l}0.667 \\
0.431\end{array}$ \\
\hline 3.0 & $\begin{array}{l}1 \\
8\end{array}$ & $\begin{array}{l}5.562 \\
6.588\end{array}$ & $\begin{array}{l}3.809 \\
4,436\end{array}$ & $\begin{array}{l}2.058 \\
2.158\end{array}$ & $\begin{array}{l}0.585 \\
0.488\end{array}$ \\
\hline 3.5 & $\frac{1}{2}$ & $\begin{array}{l}7.761 \\
6.388\end{array}$ & $\begin{array}{l}5.149 \\
4.679\end{array}$ & $\begin{array}{l}2.622 \\
1.813\end{array}$ & $\begin{array}{l}0.807 \\
0.396\end{array}$ \\
\hline 4.0 & $\frac{1}{2}$ & $\begin{array}{l}6.812 \\
6.264\end{array}$ & $\begin{array}{r}4.690 \\
4.830\end{array}$ & $\begin{array}{l}2.128 \\
1.434\end{array}$ & $\begin{array}{l}0.485 \\
0.898\end{array}$ \\
\hline 4.5 & $\frac{1}{2}$ & $\begin{array}{l}5.305 \\
3.754\end{array}$ & $\begin{array}{l}3.818 \\
3.157\end{array}$ & $\begin{array}{l}1.498 \\
0.897\end{array}$ & $\begin{array}{l}0.391 \\
0.191\end{array}$ \\
\hline 5.0 & $\frac{1}{2}$ & $\begin{array}{l}4.316 \\
4.825\end{array}$ & $\begin{array}{l}3.506 \\
4.883\end{array}$ & $\begin{array}{l}0.810 \\
0.542\end{array}$ & $\begin{array}{l}0.231 \\
0.137\end{array}$ \\
\hline 5.5 & $\begin{array}{l}1 \\
8\end{array}$ & $\begin{array}{l}6.100 \\
5.603\end{array}$ & $\begin{array}{l}5.018 \\
5.061\end{array}$ & $\begin{array}{l}1.088 \\
0.632\end{array}$ & $\begin{array}{l}0.217 \\
0.126\end{array}$ \\
\hline 6.0 & $\frac{1}{8}$ & $\begin{array}{l}5.898 \\
6.312\end{array}$ & $\begin{array}{l}5.155 \\
5.573\end{array}$ & $\begin{array}{l}0.743 \\
0.739\end{array}$ & $\begin{array}{l}0.144 \\
0.133\end{array}$ \\
\hline
\end{tabular}




\section{TABIS XD (Cont.)}

LOISTURE COMHADF DARA

$\sin 10$

\begin{tabular}{|c|c|c|c|c|c|}
\hline $\begin{array}{l}\text { Thano } \\
\text { Bres. }\end{array}$ & mory & $\begin{array}{c}\text { wt. Wot } \\
\text { Semplo } \\
\text { Come. }\end{array}$ & $\begin{array}{l}\text { wt. Dey } \\
\text { gamplo } \\
\text { Come. }\end{array}$ & $\begin{array}{c}\text { Molature } \\
\text { Loot } \\
\text { Come. }\end{array}$ & 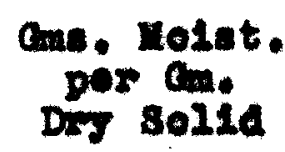 \\
\hline 6.5 & $\begin{array}{l}1 \\
8\end{array}$ & $\begin{array}{l}7.076 \\
6.800\end{array}$ & $\begin{array}{l}6.990 \\
8.098\end{array}$ & $\begin{array}{l}0.788 \\
0.841\end{array}$ & $\begin{array}{l}0.109^{\prime} \\
0.040\end{array}$ \\
\hline 7.0 & $\frac{2}{8}$ & $\begin{array}{l}4.487 \\
4.661\end{array}$ & $\begin{array}{l}4.044 \\
4.454\end{array}$ & $\begin{array}{l}0.438 \\
0.807\end{array}$ & $\begin{array}{l}0.109 \\
0.047\end{array}$ \\
\hline 7.5 & $\frac{1}{8}$ & $\begin{array}{l}5.288 \\
5.018\end{array}$ & $\begin{array}{l}4.787 \\
4.841\end{array}$ & $\begin{array}{l}0.452 \\
0.274\end{array}$ & $\begin{array}{l}0.091 \\
0.037\end{array}$ \\
\hline 8.0 & $\frac{2}{2}$ & $\begin{array}{l}6.216 \\
5.705\end{array}$ & $\begin{array}{l}6.166 \\
5.601\end{array}$ & $\begin{array}{l}0.080 \\
0.018\end{array}$ & $\begin{array}{l}0.008 \\
0.008\end{array}$ \\
\hline 8.6 & 1 & 5,480 & 5,106 & 0.374 & 0.078 \\
\hline
\end{tabular}




\section{TABLE XIYI}

\section{LOA SEMER}

Run 11

Dopertwent of Chomical Englnoering

Speed Solentiflc Sohool University of Loulsvilio

$$
\text { DRYIUO OF PROSSIAI BLUS }
$$$$
\text { Date: } 5 / 4 / 34
$$

Temperature: $120^{\circ} \mathrm{C}$.

Plant Record Data

Name: Steel blue

Bx. No.: 1006

S11p $10 .: 731$

Batoh Ho.: 4

Date struck: $3 / 29 / 34$

No. Tray Dried:

Date In: $4 / 5 / 54$
Vacuum: 26 " $\mathrm{Hg}$.

Laboratory Data

Batoh Rod.1 $4 / 30 / 34$ Batoh Rta.: $5 / 13 / 34$

Avg. Contrel Temp: $119.57^{\circ} \mathrm{C}$.

Tray Date

\begin{tabular}{|c|c|c|c|}
\hline $\begin{array}{c}\text { Tray } \\
\text { no. }\end{array}$ & $\begin{array}{l}\text { Wt of Cake } \\
\text { Before Drylns }\end{array}$ & $\begin{array}{l}\text { Wt. of Cake } \\
\text { Aftior Drying }\end{array}$ & Wt. Mo1nture \\
\hline 1 & $\begin{array}{r}6.00 \\
6.00 \\
12.00\end{array}$ & $\begin{array}{l}3.25 \\
3.00 \\
6.25\end{array}$ & $\begin{array}{l}2.75 \\
3.00 \\
5.16\end{array}$ \\
\hline
\end{tabular}


PABS XIII

CANE TEIPERATURE DL

$\operatorname{Ran} 11$

The

Fre.

0.28

0.80

0.75

2.00

1.98

1.6

2.78

2.00

2.28

2.50

8.78

8.00

8.88

8.60

3.76

4.00

4.25

4.80

4.75

5.00

5.26

8.80

6.78

6.00

6.88

6.0

6.75

7.00
Tumperatures in ${ }^{\circ} \mathrm{C}$.

Tras 1

\#2 \#8

1.

Arg.

$\# 5 \quad 76$

may 2

$33.5 \quad 31.5$

48.5

80.0

48.0

49.0

60.0

80.8

49.8

80.0

60.5

49.5

49.5

61.5

50.0

50.0

60.0

60.0

80.0

$\infty .0$

10.0

60.0

50.0

80.0

48.5

49.0

49.5

49.5

49.5

31.0

46.647 .7

$50.0 \quad 49.7$

$48.8 \quad 49.7$

49.049 .8

$49.5 \quad 50.6$

50.5

49.8

49.0

48.5

49.0

51.8

80.0

50.8

61. 7

62. 5

58.7

58.8

53.7

5..7

55.8

5.. 0

B.. 7

65.7

B5.8

55.7

56.8

56.8

56.5

59.0

58.2

35.0

48.0

50.5

80.0

50.0

58.0

85.6

53.0

59.0

61.5

61.0

68.0

68.0

62.8

68.0

68.5

60.0

63.0

64.5

67.0

71.8

78.0

31.5

4.6

80.0

18.8

48.5

50.5

61.0

50.5

50.0

50.0

50.0

80.0

60.0

60.0

80.0

60.0

50.0

60.0

57.0

67.0

85.0

25.5

$108.5107 .5 \quad 96.8$

87.0107 .5111 .5102 .0

97.0111 .5115 .5208 .0

49.5

49.0

49.0

60.8

105.0318 .5117 .5118 .7

50.5

50,6

68.5

126.0117 .5120 .011718

118.0181 .5121 .0120 .8 


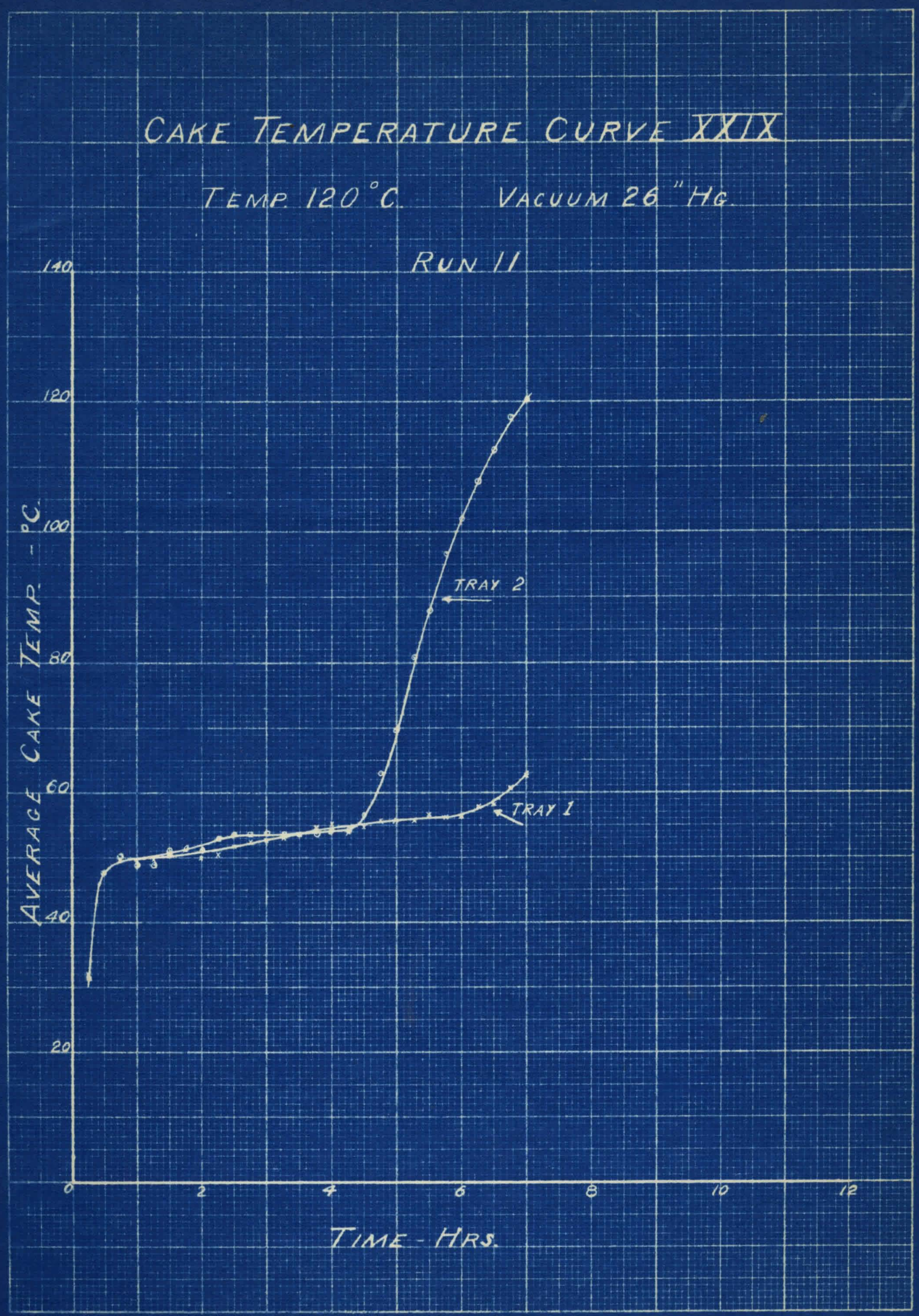


TABLE XLVIII

MOISTURE CONTENT DATA

Run 11

\begin{tabular}{|c|c|c|c|c|c|}
\hline $\begin{array}{l}\text { Time } \\
\text { Hirs. }\end{array}$ & $\begin{array}{l}\text { Tray } \\
\text { No. }\end{array}$ & $\begin{array}{l}\text { Wt. Wet } \\
\text { Sample } \\
\text { Gms. }\end{array}$ & $\begin{array}{l}\text { Wt. Dry } \\
\text { Sample } \\
\text { Cms. }\end{array}$ & $\begin{array}{l}\text { Moisture } \\
\text { Lost } \\
\text { Cms. }\end{array}$ & $\begin{array}{l}\text { Gons. Molst. } \\
\text { per Gm, } \\
\text { Dry Solid. }\end{array}$ \\
\hline 0 & $\frac{1}{2}$ & $\begin{array}{l}3.523 \\
3.741\end{array}$ & $\begin{array}{l}1.749 \\
2.066\end{array}$ & $\begin{array}{l}1.774 \\
1.675\end{array}$ & $\begin{array}{l}1.014 \\
0.810\end{array}$ \\
\hline 0.5 & $\begin{array}{l}1 \\
2\end{array}$ & $\begin{array}{l}5.148 \\
3.589\end{array}$ & $\begin{array}{l}2.508 \\
2.058\end{array}$ & $\begin{array}{l}2.640 \\
1.531\end{array}$ & $\begin{array}{l}1.052 \\
0.743\end{array}$ \\
\hline 1.0 & $\frac{1}{2}$ & $\begin{array}{l}5.171 \\
5.908\end{array}$ & $\begin{array}{l}2.823 \\
3.550\end{array}$ & $\begin{array}{l}2.348 \\
2.358\end{array}$ & $\begin{array}{l}0.831 \\
0.664\end{array}$ \\
\hline 1.5 & $\frac{1}{2}$ & $\begin{array}{l}7.224 \\
6.117\end{array}$ & $\begin{array}{l}4.014 \\
3.879\end{array}$ & $\begin{array}{l}3.210 \\
2.238\end{array}$ & $\begin{array}{l}0.800 \\
0.577\end{array}$ \\
\hline 2.0 & $\frac{1}{2}$ & $\begin{array}{l}6.199 \\
3.993\end{array}$ & $\begin{array}{l}3.617 \\
2.649\end{array}$ & $\begin{array}{l}2.582 \\
1.344\end{array}$ & $\begin{array}{l}0.779 \\
0.507\end{array}$ \\
\hline 2.5 & $\frac{1}{2}$ & $\begin{array}{l}6.178 \\
6.195\end{array}$ & $\begin{array}{l}3.586 \\
4.333\end{array}$ & $\begin{array}{l}2.592 \\
1.862\end{array}$ & $\begin{array}{l}0.722 \\
0.430\end{array}$ \\
\hline 3.0 & $\frac{1}{2}$ & $\begin{array}{l}5.992 \\
5.921\end{array}$ & $\begin{array}{l}3.756 \\
4.313\end{array}$ & $\begin{array}{l}2.236 \\
1.608\end{array}$ & $\begin{array}{l}0.595 \\
0.373\end{array}$ \\
\hline 3.5 & $\frac{1}{2}$ & $\begin{array}{l}6.637 \\
5.293\end{array}$ & $\begin{array}{l}4.336 \\
4.201\end{array}$ & $\begin{array}{l}2.301 \\
1.092\end{array}$ & $\begin{array}{l}0.532 \\
0.260\end{array}$ \\
\hline 4.0 & $\frac{1}{2}$ & $\begin{array}{l}5.800 \\
5.322\end{array}$ & $\begin{array}{l}4.552 \\
4.223\end{array}$ & $\begin{array}{l}1.248 \\
0.099\end{array}$ & $\begin{array}{l}0.274 \\
0.260\end{array}$ \\
\hline 4.5 & $\frac{1}{2}$ & $\begin{array}{l}7.056 \\
4.164\end{array}$ & $\begin{array}{l}4.995 \\
3.576\end{array}$ & $\begin{array}{l}2.061 \\
0.588\end{array}$ & $\begin{array}{l}0.412 \\
0.164\end{array}$ \\
\hline 5.0 & $\frac{1}{2}$ & $\begin{array}{l}5.511 \\
5.024\end{array}$ & $\begin{array}{l}4.085 \\
4.598\end{array}$ & $\begin{array}{l}1.426 \\
0.426\end{array}$ & $\begin{array}{l}0.349 \\
0.093\end{array}$ \\
\hline 5.5 & $\frac{1}{2}$ & $\begin{array}{l}6.019 \\
4.158\end{array}$ & $\begin{array}{l}4.720 \\
3.888\end{array}$ & $\begin{array}{l}1.299 \\
0.270\end{array}$ & $\begin{array}{l}0.275 \\
0.070\end{array}$ \\
\hline 6.0 & $\frac{1}{2}$ & $\begin{array}{l}4.401 \\
3.734\end{array}$ & $\begin{array}{l}3.609 \\
3.513\end{array}$ & $\begin{array}{l}0.792 \\
0.221\end{array}$ & $\begin{array}{l}0.220 \\
0.063\end{array}$ \\
\hline
\end{tabular}




\section{TABLE XLVIII (Cont.)}

MOISTURE CONTENT DATA

Run 11

\begin{tabular}{|c|c|c|c|c|c|}
\hline $\begin{array}{l}\text { Time } \\
\text { Hrs. }\end{array}$ & $\begin{array}{l}\text { Tray } \\
\text { No. }\end{array}$ & $\begin{array}{l}\text { wt. Wet } \\
\text { Sample } \\
\text { Cms. }\end{array}$ & $\begin{array}{l}\text { Wt. Dry } \\
\text { Sample } \\
\text { Gms. }\end{array}$ & $\begin{array}{l}\text { Molsture } \\
\text { Lost } \\
\text { Cans. }\end{array}$ & $\begin{array}{c}\text { Gns, Molst. } \\
\text { per Gm. } \\
\text { Dry Solid }\end{array}$ \\
\hline 6.5 & $\frac{1}{2}$ & $\begin{array}{l}4.383 \\
3.675\end{array}$ & $\begin{array}{l}3.716 \\
3.654\end{array}$ & $\begin{array}{l}0.667 \\
0.021\end{array}$ & $\begin{array}{l}0.180 \\
0.006\end{array}$ \\
\hline 7.0 & $\frac{1}{2}$ & $\begin{array}{l}5.339 \\
3.945\end{array}$ & $\begin{array}{l}3.665 \\
3.940\end{array}$ & $\begin{array}{l}0.674 \\
0.005\end{array}$ & $\begin{array}{l}0.144 \\
0.001\end{array}$ \\
\hline
\end{tabular}




\section{TABIS XIIX}

\section{LOQ SHEEI}

Fan 18

Department of Chomical Englnoering

speed solent1flo school Oniversity of toúlsville

\section{DRYIHO OF PRUSSIAN BLUE}

Date: $4 / 25 / 34$

Temperatare: $130^{\circ} \mathrm{C}$.

Vacuun: $26^{n} \mathrm{Hg}$.

Plant Rooord "Lata

Name: Steol Blue

Rx. No.8 4006

S11p 110.1 731

Batch Ho.: 4

Date Struck: $3 / 29 / 34$

No. Tray Dried: 5

Date In: $4 / 30 / 34$
Batch Kod.: 4/30/34

Batoh Rtd.: 5/12/34

Avg. Control Temp.: $130.25^{\circ} \mathrm{C}$.

Tray Data

- Tray Wt. of Cake

Ho. Before Drylng Ibs.

\begin{tabular}{lr}
1 & 6.75 \\
2 & 6.25 \\
\hline
\end{tabular}
Wt. of vake

After Drying 1ba.

8.75

3.25

5.00 int. Mo1sture lost

Ibs.

$$
4.00
$$

$\frac{3.00}{1.00}$ 
TABIF L

CALE TEUPERATURE DATA

Run 28

Timo

Have.

Tomperatures in ${ }^{\circ} \mathrm{C}$.

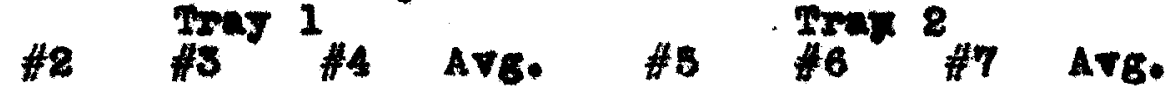

$\begin{array}{lllllllll}0.28 & 50.0 & 49.5 & 49.0 & 49.5 & 49.0 & 48.5 & 48.5 & 48.7\end{array}$

$\begin{array}{lllllllll}0.50 & 51.0 & 50.0 & 49.0 & 50.0 & 48.5 & 48.5 & 48.5 & 48.8\end{array}$

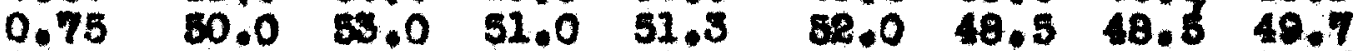

$\begin{array}{lllllllll}1.00 & 50.0 & 50.5 & 40.0 & 49.8 & 49.0 & 48.5 & 48.5 & 48.7\end{array}$

$\begin{array}{lllllllll}1.25 & 64.04 & 50.5 & 49.0 & 49.7 & 48.0 & 47.0 & 47.8 & 47.8\end{array}$

$\begin{array}{lllllllll}1.80 & 72.5 & 50.0 & 49.0 & 49.5 & 49.0 & 48.5 & 47.5 & 48.3\end{array}$

$\begin{array}{lllllllll}3.75 & 75.0 * & 80.0 & 49.0 & 49.5 & 49.0 & 48.5 & 48.5 & 48.7\end{array}$

$\begin{array}{llllllllll}8.00 & 73.0 * & 50.0 & 49.0 & 48.5 & 49.0 & 48.5 & 40.0 & 48.8\end{array}$

$\begin{array}{lllllllll}2.25 & 80.5 * & 50.0 & 49.0 & 49.5 & 49.0 & 48.5 & 48.0 & 48.5\end{array}$

$2.50 \quad 88.0 * 50.0 \quad 49.0 \quad 48.5 \quad 49.0 \quad 48.5 \quad 48.0 \quad 48.5$

2.75 82.0* $60.5 \quad 49.5 \quad 50.0 \quad 49.5 \quad 49.0 \quad 48.0 \quad 49.0$

$3.00 \quad 82.0 .50 .0 \quad 50.0 \quad 50.2$

$3.25 \quad 96.0 * 51.5 \quad 50.0 \quad 50.7$

$3.50 \quad 94.0 \% 81.0 \quad B 0.0 \quad 80.6$

$3.7562 .0 * 50.5 \quad 50.0 \quad 50.8$

$4.00 \quad 65.0 \$ 51.0 \quad 50.0 \quad 50.6$

$4.25 \quad 79.0 \% 51.0 \quad 50.0 \quad 60.5$

$4.80 \quad 80.5 \% 51.0 \quad 80.0 \quad 50.6$

$4.78 \quad 80.0+82.0 \quad 51.0 \quad 51.5$

$8.00 \quad 82.0 \% 52.0 \quad 51.051 .6$

$5.25 \quad 84.0 \% 88.0 \quad 52.0 \quad 38.6$.

$49.0 \quad 50.0 \quad 49.0 \quad 49.3$

$50.0 \quad 50.0 \quad 49.5 \quad 49.8$

$60.0 \quad 50.0 \quad 48.5 \quad 49.5$

$50.0 \quad 49.5 \quad 49.5 \quad 49.7$

$50.0 \quad 49.5 \quad 49.5 \quad 49.7$

$50.5 \quad 49.5 \quad 49.5 \quad 49.8$

$50.5 \quad 49.5 \quad 49.5 \quad 49.8$

$51.050 .0 \quad 80.0 \quad 80.3$

B8.0 $50.0 \quad 50.0 \quad 51.0$

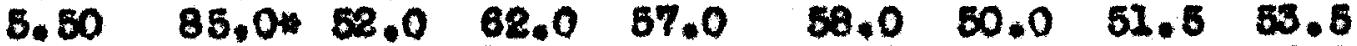

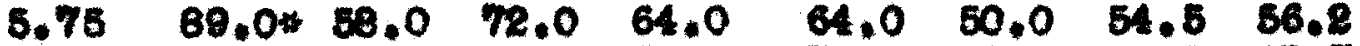

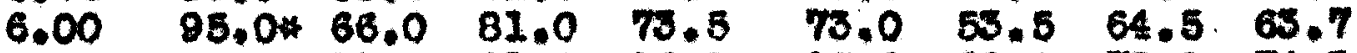

$\begin{array}{llllllll}6.28 & 96.0 * 81.0 & 91.0 & 86.0 & 86.0 & 60.0 & 78.0 & 74.3\end{array}$

$6.50 \quad 103.0 \quad 84.5 \quad 102.5 \quad 100.0 \quad 104.5 \quad 88.5 \quad 108.0 \quad 98.5$

$6.75 \quad 106.5 \quad 107.5 \quad 201.5108 .8 \quad 124.0 \quad 108.0 \quad 112.5 \quad 121.5$

$7.00 \quad 111.5 \quad 115.5 \quad 119.5 \quad 115.6 \quad 123.0 \quad 119.5 \quad 123.0 \quad 181.8$

$7.25 \quad 116.0 \quad 120.5123 .6120 .0 \quad 122.0 \quad 121.5 \quad 121.5127 .7$

$7.50 \quad 121.0 \quad 124.5 \quad 227.0$ 124.? $128.5 \quad 128.5126 .0127 .7$

$7.75 \quad 124.0124 .5 \quad 128.0 \quad 125.5 \quad 126.0 \quad 125.5 \quad 125.0 \quad 125.5$

8.00128 .0127 .5130 .0128 .5129 .0126 .5128 .5128 .0

- Value omltted in average. 
CAKE TEMPERATURE CURVE XXXT TEMr $130^{\circ} \mathrm{C}$ Vacuum $26^{\prime \prime H C}$

140
120 RUN 12

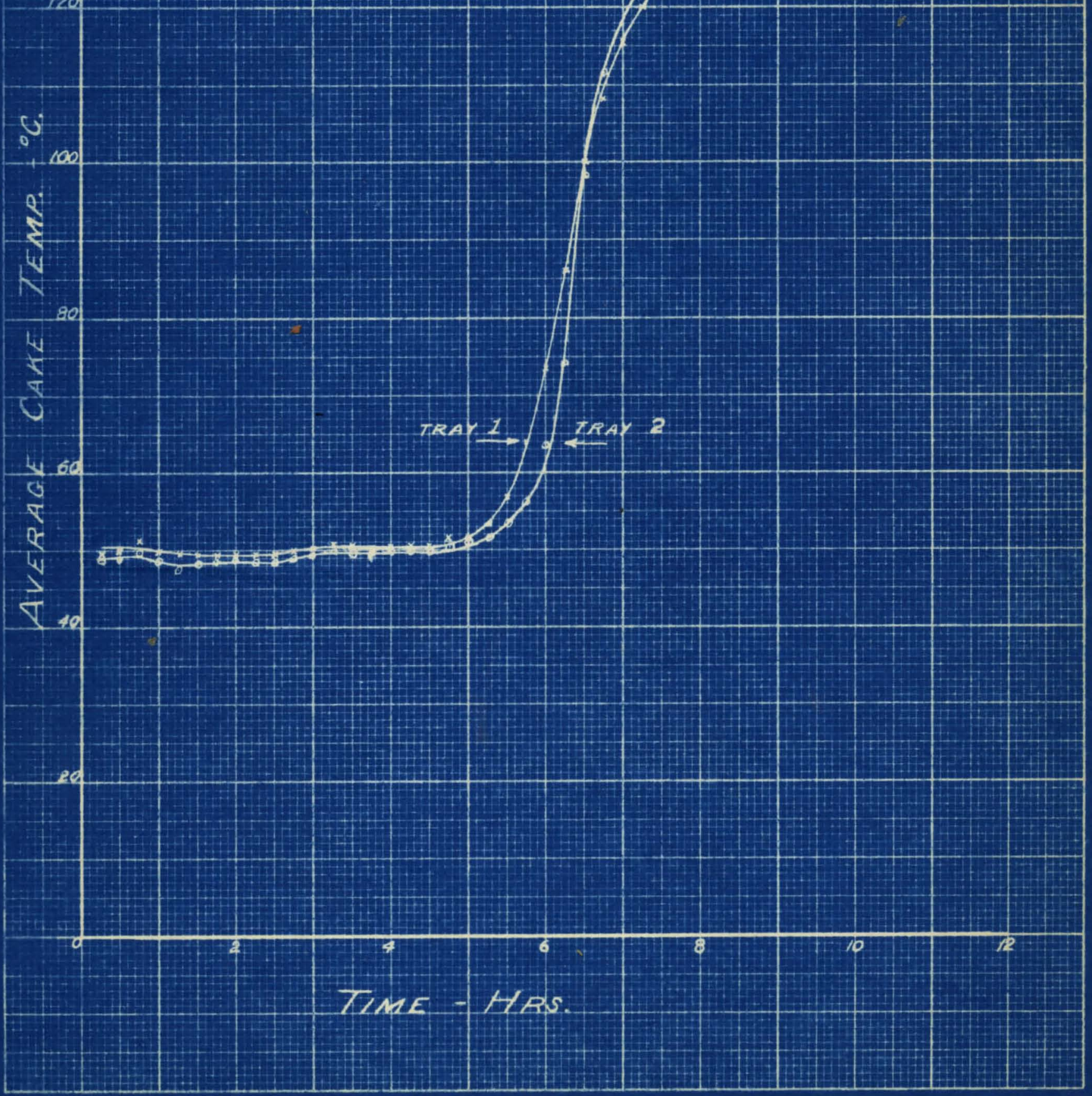


TABLE LI

MOISTURE CONTENT DATA

Run 18

\begin{tabular}{|c|c|c|c|c|c|}
\hline Time & $\begin{array}{l}\text { Trey } \\
\text { Ho. }\end{array}$ & $\begin{array}{l}\text { Wt. Wot } \\
\text { Sampio } \\
\text { Cmi . }\end{array}$ & $\begin{array}{c}\text { Wt. Dry } \\
\text { sample } \\
\text { Cans. }\end{array}$ & $\begin{array}{l}\text { Holeture } \\
\text { Lost } \\
\text { Can. }\end{array}$ & $\begin{array}{l}\text { Cono. Moset } \\
\text { per Cm. } \\
\text { Dry Solid }\end{array}$ \\
\hline$a$ & $\frac{1}{8}$ & $\begin{array}{l}6.272 \\
6.220\end{array}$ & $\begin{array}{l}2.537 \\
8.479\end{array}$ & $\begin{array}{l}2.735 \\
2.741\end{array}$ & $\begin{array}{l}1.078 \\
1.104\end{array}$ \\
\hline 0.5 & $\frac{1}{2}$ & $\begin{array}{l}4.104 \\
3.892\end{array}$ & $\begin{array}{l}2.075 \\
1.896\end{array}$ & $\begin{array}{l}8.089 \\
2.996\end{array}$ & $\begin{array}{l}0.978 \\
1.058\end{array}$ \\
\hline 1.0 & $\frac{1}{2}$ & $\begin{array}{l}4.087 \\
5,168\end{array}$ & $\begin{array}{l}8,209 \\
2.722\end{array}$ & $\begin{array}{l}1.878 \\
8.450\end{array}$ & $\begin{array}{l}0.849 \\
0.804\end{array}$ \\
\hline 1.5 & $\frac{1}{2}$ & $\begin{array}{l}4.481 \\
3.610\end{array}$ & $\begin{array}{l}2.437 \\
2.039\end{array}$ & $\begin{array}{l}2.994 \\
1.571\end{array}$ & $\begin{array}{l}0.818 \\
0.771\end{array}$ \\
\hline 2.0 & $\frac{1}{2}$ & $\begin{array}{l}4.639 \\
5.041\end{array}$ & $\begin{array}{l}2.741 \\
3.046\end{array}$ & $\begin{array}{l}1.898 \\
2.995\end{array}$ & $\begin{array}{l}0.682 \\
0.654\end{array}$ \\
\hline 2.5 & $\frac{2}{2}$ & $\begin{array}{l}6.065 \\
5.584\end{array}$ & $\begin{array}{l}3.953 \\
3.699\end{array}$ & $\begin{array}{l}2.11 .8 \\
1.825\end{array}$ & $\begin{array}{l}0.834 \\
0.493\end{array}$ \\
\hline $3.0^{-}$ & $\begin{array}{l}1 \\
2\end{array}$ & $\begin{array}{l}5.942 \\
6.047\end{array}$ & $\begin{array}{l}3.885 \\
4.250\end{array}$ & $\begin{array}{l}2.957 \\
1.797\end{array}$ & $\begin{array}{l}0.529 \\
0.428\end{array}$ \\
\hline 3.5 & $\frac{1}{2}$ & $\begin{array}{l}4.126 \\
4.618\end{array}$ & $\begin{array}{l}2.932 \\
3.532\end{array}$ & $\begin{array}{l}1.194 \\
1.096\end{array}$ & $\begin{array}{l}0.407 \\
0.308\end{array}$ \\
\hline $4.0 \mathrm{r}$ & $\begin{array}{l}1 \\
2\end{array}$ & $\begin{array}{l}4.724 \\
5.296\end{array}$ & $\begin{array}{l}3.513 \\
4.368\end{array}$ & $\begin{array}{l}1.811 \\
0.827\end{array}$ & $\begin{array}{l}0.345 \\
0.212\end{array}$ \\
\hline 4.5 & $\frac{1}{2}$ & $\begin{array}{l}5.940 \\
3.685\end{array}$ & $\begin{array}{l}4.552 \\
3.377\end{array}$ & $\begin{array}{l}1.383 \\
0.309\end{array}$ & $\begin{array}{l}0.305 \\
0.091\end{array}$ \\
\hline 5.0 & $\frac{1}{2}$ & $\begin{array}{l}3.811 \\
3.959\end{array}$ & $\begin{array}{l}2.946 \\
3.697\end{array}$ & $\begin{array}{l}0.865 \\
0.262\end{array}$ & $\begin{array}{l}0.294 \\
0.071\end{array}$ \\
\hline 5.5 & $\frac{1}{2}$ & $\begin{array}{l}4.667 \\
4.730\end{array}$ & $\begin{array}{l}3.678 \\
4.725\end{array}$ & 0.899 & 0.238 \\
\hline 6.0 & $\begin{array}{l}1 \\
2\end{array}$ & 5.001 & 4.607 & 0.394 & 0.086 \\
\hline
\end{tabular}




\section{TABIE II (Cont.) \\ MOISTURE CONIENT DATA}

$\operatorname{Ran} 18$

\begin{tabular}{|c|c|c|c|c|c|}
\hline Plmo & $\underset{\text { Noy }}{\operatorname{Tray}}$ & $\begin{array}{c}\text { Wt. Wot } \\
\text { semple } \\
\text { onie. }\end{array}$ & $\begin{array}{c}\text { Wt. Dey } \\
\text { Simple } \\
\text { Cand. }\end{array}$ & $\begin{array}{l}\text { Moleture } \\
\text { Lopt } \\
\text { Cons. }\end{array}$ & $\begin{array}{l}\text { Can. Molet. } \\
\text { Por Golid }\end{array}$ \\
\hline 6.5 & $\frac{1}{2}$ & 4,468 & 4.097 & 0.371 & $0.080^{\circ}$ \\
\hline 7.0 & $\frac{1}{8}$ & 4.265 & 4.103 & 0.078 & 0.017 \\
\hline 7.5 & $\frac{1}{2}$ & $\begin{array}{l}4.403 \\
4.641\end{array}$ & $\begin{array}{l}4.398 \\
4.641\end{array}$ & $\begin{array}{l}0.004 \\
0.000\end{array}$ & $\begin{array}{l}0.001 \\
0.000\end{array}$ \\
\hline 8.0 & $\frac{1}{2}$ & 4.386 & 4.326 & 0,000 & 0.000 \\
\hline
\end{tabular}




\section{0}


A maber of worthwhilo conclusions regarding the vecurum drying of Pruselen Blue may be drawn trom this work. It is reasonable to belleve that in general these conolusions way apply to other elmilar sollde a well.

In hi. atudy of an Inert so11d, R1dg- . way (s) found a peried of oonstant drylng rate, represented by a drying curre of ateady slope, down to molsture content of about $0.8 \mathrm{gms}$. molsture per gm. dry sel1d. From that point, the rate of drying was ohanging, as indicated by a dryling curvo of docrealing alope as tho meterlal approached dryness.

The rogults of this investigation agree with the thooretioel wolld. All of the rane porformod at elthor a temperature of $130^{\circ} \mathrm{C}$. or a vacurum of 26" Hg. how an initial poriod of conatant drying rato, followed by a falling rate period, whon the rate of drying is decreasing as the molature content approaches zero.

However, the point at whioh the firat period censes and the second commenoes la not well defined, as it varies from 0.5 to $4.0 \mathrm{gme}$. molsture 
peritgen, dry sol1d. The curves at lower temperetures and racume are nore pecullar to pruselan Blue, and 10 es in agrooment with the tbooreticed ourvee.

R1dsway (3) concluded that tho amount of racuun bad 11ttle effoct on the dry ing time, but that the time wa Invereely proportional to - the terperature.

In this invoatigation 1t was found that

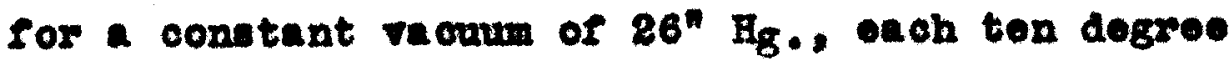
rieo in temperature docreased the drying time approximately seventeen percent, whlle tho docrease at $22^{\prime \prime}$ Hg. amounted to about nine percent per ten degree temperature increase. At 18" Hg. Inereacing the temperature has a rery notioeablo errect on the arging time, but it is alfeicult to ostimate thia, as at lower tomperaturea the twelve hour period hore used ald not bring the solid to 1t. equ1librivm moloture content.

Veourum, 11kew1se, was found to have an effect on the drylng time. At a oonatant temperature of $100^{\circ} \mathrm{C}$. a racuma of $18^{\prime \prime} \mathrm{Hg}$. did not $\mathrm{dry}$ tho $0.21 d$ in twelve hours, but racuwns of $22^{\prime \prime}$ and 
26" Fs. each brought the col1d almost to a molsture oontent of zoro in twolvo hours. At conotent tomperatures of 110,120 and $130^{\circ} \mathrm{C}$. on four 1 noh Increase in the vecuum decreased the drying time by about seventeon percent.

The curves of cake temperatures all onew tho same characteriet1ca. The cake is hoeted up to the temperature at whlch water bolls at tho presenre oxiating lanide the dryer. The terperature remalns fairlf constant at this value, unt1l, as the molsture content approechos sero, the oke temperature r1ses toward the constant control temperature. When the cake temperature levela out at tho control value, It indicates the cake is thoroughly dxy.

It 18 Interesting to observe that the time at which the cake temperature starts to rise doss not in several cases correlate with the timo at which the change from constant to falling rate of drylng occura. Instead, the cake temperature begine to rise at a molsture content of about 0.1 80. molature per gm. Ory ool1d. Thls suggest the 
probab1l1ty that the change from constant to fallIng rate of drying oocure gradualiy rather than suddenly.

It 19 observed that the arying curves at 10wer temperatures and bumiditice how "breake" - imilar in character to those of the air arying. study. However, in the cese of the veounm arying, the curre through the area of the "break" is much moothor. Thore is no abrupt cossatien and rosumption of drying, as wa found in the alf drying.

There are at th1 time no new sugges" tlow regurding the cause of those "breake", fure ther than were made in the diacusalon of the alr drying. The cause may possibly be thornal decomposition of the plgment, the audden breaking off of water of cryatalisation, or molecular roarrangement of the pigment atructure.

The fact that the "breaks" are not observed when the $\operatorname{man} 1$ is at a temperature of $130^{\circ} \mathrm{C}$.,


to the very rapld rate of drying under these cond1t10ne. If a "break" does occur at those cond1t1ons, It is of such short duration thet the mothod of sampling used did not detect $1 t$. 
Before performing tho experimontel work, 1t was suggeated that if a "broak" similar to that of the air arying occured while drying in voum, tho oake tempereture should thow a conalderable rise, due to the stopplng of the dryine, and that the tempereture of the onke would return to that of bolling water under the existing preasure as" the evaporation wa reaumod.

Th1s was ropothesized on the basis of the very abrupt "broaka" and zero rate or drylng of the alr drying invostigation. The rooulte of th1s Investigation did not produce oridence in eupport of this bypothoside. Thls is belloved due to the fact that the "brokks" found wore not abrupt in chareoter, and drying did not ontiroly conse. Although the rate of drying decreased, the evaporation contimed, and therefore the cake temporature remained at the temperature of water ovaporating at the prosaure existing within the dryer.

From the data of the Individual thormocouplo readinge it may be obserred that the cake temperature wa the same throu the ont1re cake during the greater part of the run, Indicating 
that evaporation was taking place through the whole of the eake, not at the ourface mlone. Phis date further Indicated that the top ourfaco of the cake bocume ruliy drled only very s11ghtly In advanoe of the conter and lower edgea, Whon the cake wa rully dry the tomperature rose very rapidiy to the existing dryer temperature. 
-

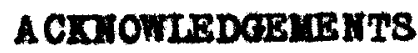


The author wishos to exprese hio eincere appreciation and thanke tos

The Paint, 011 and Varniah Club of Loulev1110, Kontuok for the award of tho Palnt, 011 and Varnish Prize for the Jear 1933-1934, whloh was largely reaponsible for makling this researob poselble, Mr. A. J. Swoder of the Kentucks Color and Chomionl Compans for his auggestion and asatences

Mossra. Clark, Bonnle and Goodell or the Iontucky Color and Chomical Compans for rumioning the plgmont,

The Tylor Sales-Fixture Compans of N1les, Mlohlgan, for the Vapor Head used in humldifyling the drying alr,

Mosare. Clifton Slaton and E. F. Musterman for their assistanoe in the exporimental work. 
BIBLTOQRAPEY 
(1) Sobnied and Ardorny "Drying of sollda. I - Praseian BIue." Thols, OnIvoralty of Loulev1110, 1983

(2) A. B. Nowmin; Trans. A. I. Ch.R., 87, 310 (1981)

(3) J. N. Rldgway; "Praot1onl Vaoum Deylng Theosy." Thesis, Dniveraity of Loule1110, 2034

(4) John H. Perry; "Chemtonl Englwer's Bandbook." MoGraw-H111 Book Comparv. 2934

(5) Lrolen Buck; Chem,Mot.Eng., 29, 686-31 (1985)

(6) H. Bohbel; Apparatobau., 34, 118-9, 235-4, 180-2; Chom, Zontr.: IN, 481 (I922)

(7) J. Dem; Arch. Salkerind., 24, 265-8 (1926)

(8) Rodgera; "Manual of Industrial Chomlatry." D. Van Nostrand Compan, 1980

(9) Minton; Toch. Assoc. Pulp Pepor Ind., I. 1 (1924)

(10) Em11 Pacaburg; Chom. Fabrik, 3, 93-5 (1930)

(11) En11 Passburg! "Apparatus for Drying in Vaouo." Brit. Patent - 3,432, Pob. 12 , 2806

(22) C. O. Lavett and D. J. Vanilarle; J. Ind. Ens. Chem. . 25, 600-8 (2981)

(13) Armand Martin; Chomlo ot Industr10, 13, 883-9 (1925)

(14) Thorp; "Outlinos of Industrial Chomistry." The Mack11Ian Compan, 1920

(15) K. A. Hofmann: Chom. Lab. Koon1gl. Akad. W18s. Muenchen. Ann., 352, 84-78

(16) E. Mueller and T. Stannisch; J. prakt Chem., 79, 81-102 
- (17) P. P. Sobmidt and B. Rageor Z. angew. Chow., 37, 334-5 (2924)

(18) O. R. Lov1, 010rn. chin. Ind. applicata., 7. 410 (1925)

(19) Juat1n-Muellex, Bulletin Soo, On1m., 49, 1885-9 (1931)

(20) E. F. Morris, J. 011 Colcur Chom. Assoo., 3. $254-60 \quad(1820)$

(21) J. R. Soott, Trane; Inst. Rubber Ind., 4. 374-95 (1829)

(22) Anon, Farbon $-\mathrm{ztg}+, 31,218-20,276-9$ (1925)

(23) W. van Hoogetraatin; Farbe. u. Lack., 23E, $244-5$ (1200)

(24) R. Sohnode; Ber. dent, bot, Oos., 41 , $343-4$ (2925)

(85) Lew1s; Ind. Eng, Chem, 13, 427 (1981)

(26) T. K. Shorwood; Ind. Bng; Chem, , 21, 1 8-16 (1929); 28, 132-56 (1930); 2, 307 (1938); 25, 311-16 (1933)

(27) Berthold Blook Chem. App., 6, 57-9 (1919)

(28) "Standard Conversion Tables for Loeds and Northrup Thermocouples." Loods and Northrup Company, Philidelphla, Pennoy 1vanle, No. 1031, Page 24 